\title{
Direct, Facile Aldehyde and Ketone $\alpha$-Selenenylation Reactions Promoted by L-Prolinamide and Pyrrolidine Sulfonamide Organocatalysts
}

\author{
Jian Wang, ${ }^{\dagger}$ Hao Li, ${ }^{\dagger}$ Yujiang Mei, ${ }^{\dagger}$ Bihshow Lou, ${ }^{\dagger,+}$ Dingguo Xu, ${ }^{\dagger}$ Daiqian Xie, ${ }^{\S}$ Hua Guo ${ }^{\dagger} *$ and \\ Wei Wang ${ }^{\dagger,} \|, *$ \\ ${ }^{\dagger}$ Department of Chemistry, University of New Mexico, Albuquerque, NM 87131 \\ ${ }^{\S}$ Department of Chemistry, Laboratory of Mesoscopic Chemistry, Institute of Theoretical and \\ Computational Chemistry, Nanjing University, Nanjing 210093, People's Republic of China \\ College of Pharmacy, East China University of Science \& Technology, P. O. Box 268, Shanghai \\ 200237, People's Republic of China
}

\section{Table of Content}

\begin{tabular}{|c|c|}
\hline Page & Description \\
\hline $\mathrm{S} 2$ & General method \\
\hline $\mathrm{S} 2$ & General procedure A for $\alpha$-selenenylation of mono- $\alpha$-substituted aldehydes \\
\hline $\mathrm{S} 2$ & $\begin{array}{l}\text { Characterization data }\left({ }^{1} \mathrm{H},{ }^{13} \mathrm{C} \text { and HRMS }\right) \text { of } \alpha \text {-selenenylation mono-substituted } \\
\text { aldehyde products for Table } 3 \text {, entries } 1-4\end{array}$ \\
\hline S3 & $\begin{array}{l}\text { Characterization data }\left({ }^{1} \mathrm{H},{ }^{13} \mathrm{C} \text { and HRMS }\right) \text { of } \alpha \text {-selenenylation mono-substituted } \\
\text { aldehyde products for Table } 3 \text {, entries } 5-8\end{array}$ \\
\hline S4 & $\begin{array}{l}\text { Characterization data }\left({ }^{1} \mathrm{H},{ }^{13} \mathrm{C} \text { and HRMS }\right) \text { of } \alpha \text {-selenenylation mono-substituted } \\
\text { aldehyde products for Table } 3 \text {, entries } 9-10\end{array}$ \\
\hline S4 & General procedure B for $\alpha$-selenenylation of $\alpha, \alpha$-dialkyl-substituted aldehydes \\
\hline S4 & $\begin{array}{l}\text { Characterization data }\left({ }^{1} \mathrm{H},{ }^{13} \mathrm{C} \text { and } \mathrm{HRMS}\right) \text { of } \alpha \text {-selenenylation } \alpha, \alpha \text {-dialkyl- } \\
\text { substituted aldehyde products for Table } 3 \text {, entries } 11 \text { and } 12\end{array}$ \\
\hline S5 & General procedure for $\alpha$-selenenylation of ketones \\
\hline S5 & $\begin{array}{l}\text { Ccharacterization data }\left({ }^{1} \mathrm{H},{ }^{13} \mathrm{C} \text { and HRMS }\right) \text { of } \alpha \text {-selenenylation ketone products } \\
\text { for Table } 6 \text {, entries } 1-3\end{array}$ \\
\hline S6 & $\begin{array}{l}\text { Characterization data }\left({ }^{1} \mathrm{H},{ }^{13} \mathrm{C} \text { and HRMS }\right) \text { of } \alpha \text {-selenenylation ketone products } \\
\text { for Table } 6 \text {, entries } 4-8\end{array}$ \\
\hline S7 & $\begin{array}{l}\text { Characterization data }\left({ }^{1} \mathrm{H},{ }^{13} \mathrm{C} \text { and HRMS }\right) \text { of } \alpha \text {-selenenylation ketone products } \\
\text { for Table } 6 \text {, entries } 9-12\end{array}$ \\
\hline S8 & $\begin{array}{l}\text { Characterization data }\left({ }^{1} \mathrm{H},{ }^{13} \mathrm{C} \text { and HRMS }\right) \text { of } \alpha \text {-selenenylation ketone products } \\
\text { for Table } 6 \text {, entries } 13-14\end{array}$ \\
\hline S8 & References \\
\hline S9-S60 & Original ${ }^{1} \mathrm{H}$ and ${ }^{13} \mathrm{C}$ NMR spectra for all $\alpha$-selenenylation products \\
\hline
\end{tabular}


General. All reactions were performed under aerobic atmosphere. Commercial, anhydrous ( $\mathrm{HPLC}$ grade) $\mathrm{CH}_{2} \mathrm{Cl}_{2}$ was used directly for reactions without further purification. HPLC grade EtOAc and hexanes were used for column chromatography. Column chromatography was performed with silica gel (230-400 mesh size). TLC plates with $\mathrm{F}_{254}$ indicator were used for monitoring reactions. The combined organic layers were dried over $\mathrm{MgSO}_{4}$. Solvents were evaporated under reduced pressure. All yields given refer to as isolated yields. ${ }^{1} \mathrm{H}$ NMR was recorded on a $500 \mathrm{MHz}$ and ${ }^{13} \mathrm{C}$ on a $125 \mathrm{MHz}$ spectrometer. HRMS experiment was performed on a high resolution magnetic sector spectrometer. Tetramethylsilane (TMS) was used as a reference for ${ }^{1} \mathrm{H}$ NMR experiments. Data for ${ }^{1} \mathrm{H}$ are reported as follows: chemical shift (ppm), and multiplicity $\left(\mathrm{s}=\right.$ singlet, $\mathrm{d}=$ doublet, $\mathrm{t}=$ triplet, $\mathrm{q}=$ quartet, $\mathrm{m}=$ multiplet). Data for ${ }^{13} \mathrm{C}$ NMR are reported as ppm.

General Procedure A for $\alpha$-Selenenylation of Aldehyde (Table 3, Entries 1-10): To a vial containing aldehyde $(0.25 \mathrm{mmol}), 0.5 \mathrm{~mL}$ of anhydrous $\mathrm{CH}_{2} \mathrm{Cl}_{2}$ and catalyst L-prolinamide 1 $(0.005 \mathrm{mmol})$ was added $N$-(phenylseleno)phthalimide $(0.3 \mathrm{mmol})$ at room temperature. After $10 \mathrm{~min}$, reaction mixture was treated with water $(5 \mathrm{~mL})$, then the solution was extracted with ethyl acetate $(3 \times 5 \mathrm{~mL})$. The combined extracts were dried over $\mathrm{MgSO}_{4}$, filtered, and concentrated in vacuo. The resulting residue was then purified by silica gel chromatography, eluting with EtOAc/Hexane to afford a clear oil.

2-(Phenylseleno)propanal: ${ }^{1}$ The reaction was carried out following the general procedure to provide a clear oil $(45 \mathrm{mg}, 81 \%) .{ }^{1} \mathrm{H}$ NMR $\left(500 \mathrm{MHz}, \mathrm{CDCl}_{3}\right): \delta 9.45(\mathrm{~d}, 1 \mathrm{H}, J=3.0 \mathrm{~Hz}), 7.51$ $(\mathrm{d}, 2 \mathrm{H}, J=7.0 \mathrm{~Hz}), 7.35$ (t, $1 \mathrm{H}, J=7.5 \mathrm{~Hz}), 7.29(\mathrm{t}, 2 \mathrm{H}, J=7.5 \mathrm{~Hz}), 3.71(\mathrm{dq}, 1 \mathrm{H}, J=7.0,3.0$ $\mathrm{Hz}), 1.46(\mathrm{~d}, 3 \mathrm{H}, J=7.0 \mathrm{~Hz}) ;{ }^{13} \mathrm{C}$ NMR $\left(125 \mathrm{MHz}, \mathrm{CDCl}_{3}\right): \delta 193.7,136.3,129.5,129.1,125.9$, 46.8, 13.6. HRMS (EI) calcd for $\mathrm{C}_{9} \mathrm{H}_{10} \mathrm{OSe}\left(\mathrm{M}^{+}\right)$213.9891, obsd 213.9909 .<smiles>CC(C=O)C(=O)c1ccccc1</smiles>

2-(Phenylseleno)butyraldehyde: ${ }^{1}$ The reaction was carried out following the general procedure to provide a clear oil $(49 \mathrm{mg}, 83 \%) .{ }^{1} \mathrm{H}$ NMR $\left(500 \mathrm{MHz}, \mathrm{CDCl}_{3}\right): \delta 9.41(\mathrm{~d}, 1 \mathrm{H}, J=3.5 \mathrm{~Hz})$, $7.51(\mathrm{~d}, 2 \mathrm{H}, J=7.0 \mathrm{~Hz}), 7.34(\mathrm{t}, 1 \mathrm{H}, J=7.5 \mathrm{~Hz}), 7.28(\mathrm{t}, 2 \mathrm{H}, J=7.5 \mathrm{~Hz}), 3.71(\mathrm{dt}, 1 \mathrm{H}, J=7.5$, $3.5 \mathrm{~Hz}), 1.91-1.83(\mathrm{~m}, 1 \mathrm{H}), 1.75-1.58(\mathrm{~m}, 1 \mathrm{H}), 1.08(\mathrm{t}, 3 \mathrm{H}, J=7.5 \mathrm{~Hz}) ;{ }^{13} \mathrm{C}$ NMR $(125 \mathrm{MHz}$, $\mathrm{CDCl}_{3}$ ): $\delta 193.3,136.1,129.5,129.0,126.1,54.9,21.3,12.8$. HRMS (EI) calcd for $\mathrm{C}_{10} \mathrm{H}_{12} \mathrm{OSe}$ $\left(\mathrm{M}^{+}\right)$228.0048, obsd 228.0065.<smiles>CCC(Sc1ccccc1)C(=O)c1ccccc1</smiles>

2-(Phenylseleno)pentanal: ${ }^{1}$ The reaction was carried out following the general procedure to provide a clear oil $(53 \mathrm{mg}, 85 \%)$. ${ }^{1} \mathrm{H}$ NMR $\left(500 \mathrm{MHz}, \mathrm{CDCl}_{3}\right): \delta 9.39(\mathrm{~d}, 1 \mathrm{H}, J=3.5 \mathrm{~Hz}), 7.51$ $(\mathrm{d}, 2 \mathrm{H}, J=7.0 \mathrm{~Hz}), 7.33(\mathrm{t}, 1 \mathrm{H}, J=7.5 \mathrm{~Hz}), 7.28(\mathrm{t}, 2 \mathrm{H}, J=7.5 \mathrm{~Hz}), 3.61(\mathrm{dt}, 1 \mathrm{H}, J=7.5,3.5 \mathrm{~Hz}$ ), 1.79-1.78 (m, 1H), 1.70-1.67 (m, 1H), 1.65-1.42 (m, 2H), $0.95(\mathrm{t}, 3 \mathrm{H}, J=7.5 \mathrm{~Hz}) ;{ }^{13} \mathrm{C}$ NMR $\left(125 \mathrm{MHz}, \mathrm{CDCl}_{3}\right): \delta 193.3,136.0,129.5,129.0,126.2,52.9,29.9,21.4,13.9$. HRMS (EI) calcd for $\mathrm{C}_{11} \mathrm{H}_{14} \mathrm{OSe}\left(\mathrm{M}^{+}\right)$242.0204, obsd 242.0191. 
<smiles>CCCC(C=O)Sc1ccccc1</smiles>

3-Methyl-2-(Phenylseleno)butyraldehyde: ${ }^{1}$ The reaction was carried out following the general procedure to provide a clear oil $(55 \mathrm{mg}, 88 \%)$. ${ }^{1} \mathrm{H}$ NMR $\left(500 \mathrm{MHz}, \mathrm{CDCl}_{3}\right): \delta 9.36(\mathrm{~d}, 1 \mathrm{H}, J=$ $5.0 \mathrm{~Hz}), 7.51(\mathrm{~d}, 2 \mathrm{H}, J=7.0 \mathrm{~Hz}), 7.32(\mathrm{t}, 1 \mathrm{H}, J=7.5 \mathrm{~Hz}), 7.28(\mathrm{t}, 2 \mathrm{H}, J=7.5 \mathrm{~Hz}), 3.61(\mathrm{dd}, 1 \mathrm{H}$, $J=9.0,5.0 \mathrm{~Hz}), 2.12-2.05(\mathrm{~m}, 1 \mathrm{H}), 1.20(\mathrm{~d}, 3 \mathrm{H}, J=6.5 \mathrm{~Hz}), 1.09(\mathrm{~d}, 3 \mathrm{H}, J=7.0 \mathrm{~Hz})$; ${ }^{13} \mathrm{C} \mathrm{NMR}$ $\left(125 \mathrm{MHz}, \mathrm{CDCl}_{3}\right): \delta 192.8,135.6,129.5,128.8,127.0,62.1,27.4,21.5$ 21.3. HRMS (EI) calcd for $\mathrm{C}_{11} \mathrm{H}_{14} \mathrm{OSe}\left(\mathrm{M}^{+}\right)$242.0204, obsd 242.0191.<smiles>CC(C)C(C=O)C(=O)c1ccccc1</smiles>

2-(Phenylseleno)hexanal: ${ }^{1}$ The reaction was carried out following the general procedure to provide a clear oil $(50 \mathrm{mg}, 78 \%)$. ${ }^{1} \mathrm{H} \mathrm{NMR}\left(500 \mathrm{MHz}, \mathrm{CDCl}_{3}\right): \delta 9.38(\mathrm{~d}, 1 \mathrm{H}, J=3.5 \mathrm{~Hz}), 7.50$ $(\mathrm{d}, 2 \mathrm{H}, J=7.0 \mathrm{~Hz}), 7.33(\mathrm{t}, 1 \mathrm{H}, J=7.0 \mathrm{~Hz}), 7.28(\mathrm{t}, 2 \mathrm{H}, J=7.5 \mathrm{~Hz}), 3.61(\mathrm{dt}, 1 \mathrm{H}, J=7.5,3.5 \mathrm{~Hz}$ ), $1.87-1.80(\mathrm{~m}, 1 \mathrm{H}), 1.71-1.64(\mathrm{~m}, 1 \mathrm{H}), 1.54-1.48(\mathrm{~m}, 1 \mathrm{H}), 1.43-1.30(\mathrm{~m}, 3 \mathrm{H}), 0.90(\mathrm{t}, 3 \mathrm{H}, J=$ $7.5 \mathrm{~Hz}) ;{ }^{13} \mathrm{C} \mathrm{NMR}\left(125 \mathrm{MHz}, \mathrm{CDCl}_{3}\right): \delta 193.3,136.0,129.5,129.0,126.2,53.1,30.3,27.6$, 22.6, 14.1. HRMS (EI) calcd for $\mathrm{C}_{12} \mathrm{H}_{16} \mathrm{OSe}\left(\mathrm{M}^{+}\right)$256.0361, obsd 256.0323.<smiles>CCCCC(C=O)Sc1ccccc1</smiles>

2-(Phenylseleno)heptanal: ${ }^{2}$ The reaction was carried out following the general procedure to provide a clear oil $(58 \mathrm{mg}, 86 \%) .{ }^{1} \mathrm{H} \mathrm{NMR}\left(500 \mathrm{MHz}, \mathrm{CDCl}_{3}\right): \delta 9.38(\mathrm{~d}, 1 \mathrm{H}, J=4.0 \mathrm{~Hz}), 7.50$ $(\mathrm{dd}, 2 \mathrm{H}, J=8.0,1.0 \mathrm{~Hz}), 7.35-7.26(\mathrm{~m}, 3 \mathrm{H}), 3.60(\mathrm{dt}, 1 \mathrm{H}, J=7.0,4.0 \mathrm{~Hz}), 1.86-1.80(\mathrm{~m}, 1 \mathrm{H})$, $1.78-1.63(\mathrm{~m}, 1 \mathrm{H}), 1.55-1.45(\mathrm{~m}, 1 \mathrm{H}), 1.46-1.36(\mathrm{~m}, 1 \mathrm{H}), 1.35-1.26(\mathrm{~m}, 4 \mathrm{H}), 0.89(\mathrm{t}, 3 \mathrm{H}, J=7.0$ $\mathrm{Hz}) ;{ }^{13} \mathrm{C}$ NMR $\left(125 \mathrm{MHz}, \mathrm{CDCl}_{3}\right): \delta 193.3,136.0,129.5,129.0,126.0,53.2,31.6,27.9,27.8$, 22.6, 14.1. HRMS (EI) calcd for $\mathrm{C}_{13} \mathrm{H}_{18} \mathrm{OSe}\left(\mathrm{M}^{+}\right)$270.0517, obsd 270.0527.<smiles>CCCCCC([SbH])c1ccccc1</smiles>

2-(Phenylseleno)octanal: ${ }^{3}$ The reaction was carried out following the general procedure to provide a clear oil $(67 \mathrm{mg}, 95 \%)$. ${ }^{1} \mathrm{H} \mathrm{NMR}\left(500 \mathrm{MHz}, \mathrm{CDCl}_{3}\right): \delta 9.38(\mathrm{~d}, 1 \mathrm{H}, J=3.5 \mathrm{~Hz}), 7.50$ $(\mathrm{d}, 2 \mathrm{H}, J=7.5 \mathrm{~Hz}), 7.33(\mathrm{t}, 1 \mathrm{H}, J=7.5 \mathrm{~Hz}), 7.28(\mathrm{t}, 2 \mathrm{H}, J=7.5 \mathrm{~Hz}), 3.60(\mathrm{dt}, 1 \mathrm{H}, J=7.0,3.5 \mathrm{~Hz}$ ), 1.86-1.79 (m, 1H), 1.71-1.66 (m, 1H), 1.58-1.47 (m, 1H), 1.45-1.25 (m, 7H), 0.88 (t, 3H, J= $7.0 \mathrm{~Hz}) ;{ }^{13} \mathrm{C} \mathrm{NMR}\left(125 \mathrm{MHz}, \mathrm{CDCl}_{3}\right): \delta 193.3,136.0,129.5,129.0,126.2,53.2,31.8,29.1$, 28.1, 27.9, 22.7, 14.2. HRMS (EI) calcd for $\mathrm{C}_{14} \mathrm{H}_{20} \mathrm{OSe}\left(\mathrm{M}^{+}\right)$284.0674, obsd 284.0685.<smiles>CCCCCCC(C=O)Sc1ccccc1</smiles>

2-(Phenylseleno)nonanal: The reaction was carried out following the general procedure to provide a clear oil $(67 \mathrm{mg}, 91 \%) .{ }^{1} \mathrm{H} \mathrm{NMR}\left(500 \mathrm{MHz}, \mathrm{CDCl}_{3}\right): \delta 9.38(\mathrm{~d}, 1 \mathrm{H}, J=4.0 \mathrm{~Hz}), 7.50$ 
$(\mathrm{d}, 2 \mathrm{H}, J=7.0 \mathrm{~Hz}), 7.33(\mathrm{t}, 1 \mathrm{H}, J=7.5 \mathrm{~Hz}), 7.28(\mathrm{t}, 2 \mathrm{H}, J=7.0 \mathrm{~Hz}), 3.60(\mathrm{dt}, 1 \mathrm{H}, J=7.0,3.5 \mathrm{~Hz}$ ), 1.86-1.79 (m, 1H), 1.71-1.64 (m, 1H), 1.57-1.47 (m, 1H), 1.43-1.36 (m, 1H), 1.35-1.26 (m., $8 \mathrm{H}), 0.88(\mathrm{t}, 3 \mathrm{H}, J=7.0 \mathrm{~Hz}) ;{ }^{13} \mathrm{C}$ NMR $\left(125 \mathrm{MHz}, \mathrm{CDCl}_{3}\right): \delta 193.3,136.0,129.5,129.0,126.2$, 53.2, 31.9, 29.4, 29.2, 28.2, 27.8, 22.8, 14.3. HRMS (EI) calcd for $\mathrm{C}_{15} \mathrm{H}_{22} \mathrm{OSe}\left(\mathrm{M}^{+}\right)$298.0830, obsd 298.0807.<smiles>CCCCCCCC(C=O)Sc1ccccc1</smiles>

2-(Phenylseleno)decanal: ${ }^{4}$ The reaction was carried out following the general procedure to provide a clear oil $(67 \mathrm{mg}, 84 \%)$. ${ }^{1} \mathrm{H} \mathrm{NMR}\left(500 \mathrm{MHz}, \mathrm{CDCl}_{3}\right): \delta 9.38(\mathrm{~d}, 1 \mathrm{H}, J=4.0 \mathrm{~Hz}), 7.50$ $(\mathrm{d}, 2 \mathrm{H}, J=7.0 \mathrm{~Hz}), 7.33(\mathrm{t}, 1 \mathrm{H}, J=7.5 \mathrm{~Hz}), 7.28(\mathrm{t}, 2 \mathrm{H}, J=7.5 \mathrm{~Hz}), 3.60(\mathrm{dt}, 1 \mathrm{H}, J=7.0,4.0 \mathrm{~Hz}$ ), 1.86-1.79 (m, 1H), 1.71-1.64 (m, 1H), 1.57-1.47 (m, 1H), 1.43-1.37 (m, 1H), 1.31-1.25 (m., $10 \mathrm{H}), 0.88(\mathrm{t}, 3 \mathrm{H}, J=7.0 \mathrm{~Hz}) ;{ }^{13} \mathrm{C}$ NMR $\left(125 \mathrm{MHz}, \mathrm{CDCl}_{3}\right): \delta 193.3,136.0,129.5,129.0,126.2$, 53.2, 32.0, 29.5, 29.4, 29.3, 28.2, 27.8, 22.8, 14.3. HRMS (EI) calcd for $\mathrm{C}_{16} \mathrm{H}_{24} \mathrm{OSe}\left(\mathrm{M}^{+}\right)$ 312.0987 , obsd 312.0974 .<smiles>CCCCCCCCC(C=O)Sc1ccccc1</smiles>

3-Phenyl-2-(Phenylseleno)propionaldehyde: ${ }^{1}$ The reaction was carried out following the general procedure to provide a clear oil $(60 \mathrm{mg}, 80 \%) .{ }^{1} \mathrm{H} \mathrm{NMR}\left(500 \mathrm{MHz}, \mathrm{CDCl}_{3}\right): \delta 9.47$ (d, $1 \mathrm{H}, J=4.0 \mathrm{~Hz}), 7.49(\mathrm{~d}, 2 \mathrm{H}, J=7.5 \mathrm{~Hz}), 7.35(\mathrm{t}, 1 \mathrm{H}, J=7.0 \mathrm{~Hz}), 7.31-7.19(\mathrm{~m}, 7 \mathrm{H}), 3.90-3.87$ $(\mathrm{m}, 1 \mathrm{H}), 3.23(\mathrm{dd}, 1 \mathrm{H}, J=14.0,8.0 \mathrm{~Hz}), 3.00(\mathrm{dd}, 1 \mathrm{H}, J=14.0,6.5 \mathrm{~Hz}) ;{ }^{13} \mathrm{C} \mathrm{NMR}(125 \mathrm{MHz}$, $\mathrm{CDCl}_{3}$ ): $\delta 129.4,138.5,136.3,129.6,129.2,128.8,127.1,126.0,53.7,34.3$. HRMS (EI) calcd for $\mathrm{C}_{15} \mathrm{H}_{14} \mathrm{OSe}\left(\mathrm{M}^{+}\right)$290.0204, obsd 290.0183.<smiles>O=CC([SeH])Cc1ccccc1</smiles>

General Procedure B for $\alpha$-Selenenylation of Aldehyde (Table 3, Entries 11 and 12): To a vial containing an aldehyde $(0.25 \mathrm{mmol})$, and $0.5 \mathrm{~mL}$ of anhydrous $\mathrm{CH}_{2} \mathrm{Cl}_{2}$ was added catalyst Lprolinamide I $(0.005 \mathrm{mmol})$ at room temperature. The mixture was vigorously stirred for $0.5 \mathrm{~h}$ in the presence of $4 \AA$ molecule sieves $(40 \mathrm{mg})$. Then $N$-(phenylseleno)phthalimide $(0.3 \mathrm{mmol})$ was added. After $0.5 \mathrm{~h}$, the molecule sieves were removed by filtrating paper and then the filtrate was treated with water $(5 \mathrm{~mL})$, the solution was extracted with ethyl acetate $(3 \times 5 \mathrm{~mL})$. The combined extracts were dried over $\mathrm{MgSO}_{4}$, filtered, and concentrated in vacuo. The resulting residue was then purified by silica gel chromatography, eluting with EtOAc/Hexane $(1 / 40)$ to provide a clear oil.

2-Methyl-2-(Phenylseleno)propionaldehyde: ${ }^{5}$ The reaction was carried out following the general procedure to provide a clear oil $(45 \mathrm{mg}, 76 \%) .{ }^{1} \mathrm{H} \mathrm{NMR}\left(500 \mathrm{MHz}, \mathrm{CDCl}_{3}\right): \delta 9.26(\mathrm{~s}$, $1 \mathrm{H}), 7.49(\mathrm{~d}, 2 \mathrm{H}, J=7.0 \mathrm{~Hz}), 7.39(\mathrm{t}, 1 \mathrm{H}, J=7.5 \mathrm{~Hz}), 7.30(\mathrm{t}, 2 \mathrm{H}, J=8.0 \mathrm{~Hz}), 1.44(\mathrm{~s}, 6 \mathrm{H}) ;{ }^{13} \mathrm{C}$ NMR (125 MHz, $\left.\mathrm{CDCl}_{3}\right): \delta 193.7,138.0,129.7,129.3,126.3,53.6,21.7$. HRMS (EI) calcd for $\mathrm{C}_{10} \mathrm{H}_{12} \mathrm{OSe}\left(\mathrm{M}^{+}\right)$228.0048, obsd 228.0065. 
<smiles>CC(C)([SeH])C=O</smiles>

1-(Phenylseleno)cyclohexanecarbaldehyde: ${ }^{1}$ The reaction was carried out following the general procedure to provide a clear oil $(56 \mathrm{mg}, 81 \%) .{ }^{1} \mathrm{H} \mathrm{NMR}\left(500 \mathrm{MHz}, \mathrm{CDCl}_{3}\right): \delta 9.18(\mathrm{~s}$, $1 \mathrm{H}), 7.47(\mathrm{~d}, 2 \mathrm{H}, J=7.0 \mathrm{~Hz}), 7.37(\mathrm{t}, 1 \mathrm{H}, J=7.5 \mathrm{~Hz}), 7.30(\mathrm{t}, 2 \mathrm{H}, J=7.5 \mathrm{~Hz}), 1.92-1.88(\mathrm{~m}$, $2 \mathrm{H}), 1.76-1.71(\mathrm{~m}, 4 \mathrm{H}), 1.56-1.53(\mathrm{~m}, 1 \mathrm{H}), 1.45-1.23(\mathrm{~m}, 3 \mathrm{H}) ;{ }^{13} \mathrm{C} \mathrm{NMR}\left(125 \mathrm{MHz}, \mathrm{CDCl}_{3}\right): \delta$ 192.8, 138.1, 129.6, 129.2, 125.2, 59.9, 30.9, 25.7, 24.2. HRMS (EI) calcd for $\mathrm{C}_{13} \mathrm{H}_{16} \mathrm{OSe}\left(\mathrm{M}^{+}\right)$ 268.0361 , obsd 268.0336.<smiles>O=CC1([SeH]c2ccccc2)CCCCC1</smiles>

General Procedure for $\alpha$-Selenenylation of Ketones (Table 6, entries 1-14): To a vial containing ketone $(0.3 \mathrm{mmol})$, and $1.0 \mathrm{~mL}$ of anhydrous $\mathrm{CH}_{2} \mathrm{Cl}_{2}$ was added catalyst pyrrolidine trifluoromethanesulfonamide $\mathbf{I}(0.03 \mathrm{mmol})$ at room temperature. The mixture was vigorously stirred for $1 \mathrm{hr}$ before $\mathrm{N}$-(phenylseleno)phthalimide $(0.3 \mathrm{mmol})$ was added. After $16-48 \mathrm{~h}$, the reaction mixture was treated with water $(10 \mathrm{~mL})$, and then the solution was extracted with ethyl acetate $(3 \times 10 \mathrm{~mL})$. The combined extracts were dried over $\mathrm{MgSO}_{4}$, filtered, and concentrated in vacuo. The resulting residue was then purified by silica gel chromatography and fractions were collected and concentrated in vacuo to provide a clear oil.

1-(Phenylselanyl)propan-2-one: ${ }^{5}$ The title compound was prepared according to the general procedure in $69 \%$ yield. ${ }^{1} \mathrm{H}$ NMR $\left(500 \mathrm{MHz}, \mathrm{CDCl}_{3}\right): \delta 7.55-7.50(\mathrm{~m}, 2 \mathrm{H}), 7.26-7.31(\mathrm{~m}, 3 \mathrm{H})$, 3.59 (s, 2H), 2.27 (s, 3H); ${ }^{13} \mathrm{C} \mathrm{NMR}\left(125 \mathrm{MHz}, \mathrm{CDCl}_{3}\right): \delta 203.7,33.5,129.6,128.9,128.2,37.0$, 28.2; HRMS (EI) calcd for $\mathrm{C}_{9} \mathrm{H}_{10} \mathrm{OSe}\left(\mathrm{M}^{+}\right)$213.9891, obsd 213.9909.<smiles>CC(=O)C[SeH]c1ccccc1</smiles>

2-(Phenylselanyl)pentan-3-one: ${ }^{5}$ The title compound was prepared according to the general procedure in $61 \%$ yield. ${ }^{1} \mathrm{H} \mathrm{NMR}\left(500 \mathrm{MHz}, \mathrm{CDCl}_{3}\right): \delta 7.52(\mathrm{dd}, 2 \mathrm{H}, J=8.0,1.0 \mathrm{~Hz}), 7.37-7.25$ $(\mathrm{m}, 3 \mathrm{H}), 3.81(\mathrm{q}, 1 \mathrm{H}, J=7.0 \mathrm{~Hz}), 2.78(\mathrm{dq}, 1 \mathrm{H}, J=17.5,7.0 \mathrm{~Hz}), 2.50(\mathrm{dq}, 1 \mathrm{H}, J=17.5,7.0$ $\mathrm{Hz}), 1.48(\mathrm{~d}, 3 \mathrm{H}, J=7.0 \mathrm{~Hz}), 1.08(\mathrm{t}, 3 \mathrm{H}, J=7.5 \mathrm{~Hz}) ;{ }^{13} \mathrm{C} \mathrm{NMR}\left(125 \mathrm{MHz}, \mathrm{CDCl}_{3}\right): \delta 207.9$, 136.0, 129.4, 128.9, 127.4, 45.3, 33.2, 16.7, 8.6; HRMS (EI) calcd for $\mathrm{C}_{11} \mathrm{H}_{14} \mathrm{OSe}\left(\mathrm{M}^{+}\right)$ 242.0204 , obsd 242.0191.<smiles>CCC(=O)C(C)[SeH]c1ccccc1</smiles> 
3-(Phenylselanyl)heptan-4-one: ${ }^{6}$ The title compound was prepared according to the general procedure in $58 \%$ yield. ${ }^{1} \mathrm{H}$ NMR ( $\left.500 \mathrm{MHz}, \mathrm{CDCl}_{3}\right): \delta 7.51(\mathrm{dd}, 2 \mathrm{H}, J=7.5,1.0 \mathrm{~Hz}), 7.35-7.25$ (m, 3H), $3.56(\mathrm{t}, 1 \mathrm{H}, J=7.5 \mathrm{~Hz}), 2.63(\mathrm{dt}, 1 \mathrm{H}, J=16.5,7.5 \mathrm{~Hz}), 2.49$ (dq, $1 \mathrm{H}, J=16.5,7.0 \mathrm{~Hz})$, $1.91-1.85(\mathrm{~m}, 1 \mathrm{H}), 1.76-1.70(\mathrm{~m}, 1 \mathrm{H}), 1.65-1.58(\mathrm{~m}, 1 \mathrm{H}), 0.98(\mathrm{~d}, 3 \mathrm{H},=7.5 \mathrm{~Hz}), 0.91(\mathrm{t}, 3 \mathrm{H}, J=$ $7.5 \mathrm{~Hz}) ;{ }^{13} \mathrm{C}$ NMR $\left(125 \mathrm{MHz}, \mathrm{CDCl}_{3}\right): \delta 206.6,135.8,129.3,128.7,127.6,53.7,42.6,23.9$, 17.8, 13.9, 13.0; HRMS (EI) calcd for $\mathrm{C}_{13} \mathrm{H}_{18} \mathrm{OSe}\left(\mathrm{M}^{+}\right)$270.0517, obsd 270.0520.<smiles>CCCC(=O)C(CC)[SeH]c1ccccc1</smiles>

4-Methyl-1-(phenylselanyl)pentan-2-one: The title compound was prepared according to the general procedure in $62 \%$ yield. ${ }^{1} \mathrm{H}$ NMR $\left(500 \mathrm{MHz}, \mathrm{CDCl}_{3}\right): \delta 7.53$ (dd, $\left.2 \mathrm{H}, J=6.0,3.0 \mathrm{~Hz}\right)$, 7.29-7.26 (m, 3H), 3.58 (s, 2H), 2.46 (d, $2 \mathrm{H}, J=7.0 \mathrm{~Hz}), 2.13-2.08(\mathrm{~m}, 1 \mathrm{H}), 0.89$ (d, $6 \mathrm{H}, J=6.5$ $\mathrm{Hz}) ;{ }^{13} \mathrm{C}$ NMR $\left(125 \mathrm{MHz}, \mathrm{CDCl}_{3}\right): \delta 205.6,133.5,129.5,129.1,128.1,49.9,36.6,24.9,22.7$; HRMS (EI) calcd for $\mathrm{C}_{12} \mathrm{H}_{16} \mathrm{OSe}\left(\mathrm{M}^{+}\right)$256.0361, obsd 256.0323.<smiles>CC(C)CC(=O)C[SeH]</smiles>

1-(Phenylselanyl)hex-5-en-2-one: ${ }^{7}$ The title compound was prepared according to the general procedure in $63 \%$ yield. ${ }^{1} \mathrm{H}$ NMR $\left(500 \mathrm{MHz}, \mathrm{CDCl}_{3}\right): \delta 7.53$ (dd, $\left.2 \mathrm{H}, J=6.0,2.0 \mathrm{~Hz}\right), 7.35-7.26$ (m, 3H), 5.80-5.73 (m, 1H), 5.02-4.90 (m, 2H), 3.59 (s, 2H), $2.68(\mathrm{t}, 2 \mathrm{H}, J=7.0 \mathrm{~Hz}), 2.33-2.29$ (m, 2H); ${ }^{13} \mathrm{C}$ NMR $\left(125 \mathrm{MHz}, \mathrm{CDCl}_{3}\right): \delta 205.1,137.1,136.1,133.6,129.5,128.2,115.6,40.0$, 36.3, 28.2; HRMS (EI) calcd for $\mathrm{C}_{12} \mathrm{H}_{14} \mathrm{OSe}\left(\mathrm{M}^{+}\right)$254.0204, obsd 254.0280.

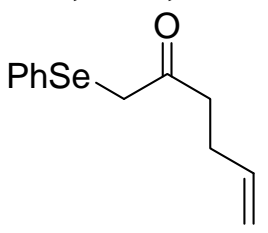

1-Phenyl-2-(phenylselanyl)ethanone: ${ }^{8}$ The title compound was prepared according to the general procedure in $81 \%$ yield. ${ }^{1} \mathrm{H}$ NMR $\left(500 \mathrm{MHz}, \mathrm{CDCl}_{3}\right): \delta 7.88(\mathrm{dd}, 2 \mathrm{H}, J=8.0,1.0 \mathrm{~Hz})$, 7.56-7.52 (m, 3H), $7.42(\mathrm{t}, 2 \mathrm{H}, J=7.5 \mathrm{~Hz}), 7.29-7.24(\mathrm{~m}, 3 \mathrm{H}), 4.17(\mathrm{~s}, 2 \mathrm{H}) ;{ }^{13} \mathrm{C}$ NMR $(125$ $\left.\mathrm{MHz}, \mathrm{CDCl}_{3}\right): \delta 195.1,135.6,134.2,133.5,129.4,129.2,128.9,128.8,128.3,32.9$; HRMS (EI) calcd for $\mathrm{C}_{14} \mathrm{H}_{12} \mathrm{OSe}\left(\mathrm{M}^{+}\right)$276.0048, obsd 276.0064.<smiles>O=C(C[Se]c1ccccc1)c1ccccc1</smiles>

2-(Phenylselanyl)cyclopentanone: ${ }^{5}$ The title compound was prepared according to the general procedure in $78 \%$ yield. ${ }^{1} \mathrm{H}$ NMR $\left(500 \mathrm{MHz}, \mathrm{CDCl}_{3}\right): \delta 7.51(\mathrm{dd}, 2 \mathrm{H}, J=6.5,1.5 \mathrm{~Hz}), 7.34-7.26$ $(\mathrm{m}, 3 \mathrm{H}), 3.75(\mathrm{t}, 1 \mathrm{H}, J=7.5 \mathrm{~Hz}), 2.36-2.28(\mathrm{~m}, 2 \mathrm{H}), 2.22-2.15(\mathrm{~m}, 1 \mathrm{H}), 2.08-1.89(\mathrm{~m}, 3 \mathrm{H}) ;{ }^{13} \mathrm{C}$ NMR (125 MHz, $\left.\mathrm{CDCl}_{3}\right): \delta 214.7,135.5,129.3,128.6,128.0,46.6,36.5,30.9,21.1$; HRMS (EI) calcd for $\mathrm{C}_{11} \mathrm{H}_{12} \mathrm{OSe}\left(\mathrm{M}^{+}\right) 240.0048$, obsd 240.0062 . 
<smiles>O=C1CCCC1Sc1ccccc1</smiles>

2,2-Dimethyl-5-(phenylselanyl)cyclopentanone: The title compound was prepared according to the general procedure in $67 \%$ yield. ${ }^{1} \mathrm{H}$ NMR $\left(500 \mathrm{MHz}, \mathrm{CDCl}_{3}\right): \delta 7.61(\mathrm{dd}, 2 \mathrm{H}, J=6.0,1.0$ $\mathrm{Hz})$, 7.34-7.26 (m, 3H), 3.83 (dd, $1 \mathrm{H}, J=7.5,5.0 \mathrm{~Hz}), 2.32-2.25(\mathrm{~m}, 1 \mathrm{H}), 1.96-1.89(\mathrm{~m}, 2 \mathrm{H})$, $1.78-1.73(\mathrm{~m}, 1 \mathrm{H}), 1.03(\mathrm{~s}, 3 \mathrm{H}), 1.02(\mathrm{~s}, 3 \mathrm{H}) ;{ }^{13} \mathrm{C} \mathrm{NMR}\left(125 \mathrm{MHz}, \mathrm{CDCl}_{3}\right): \delta 218.2,135.6$, 129.0, 128.4, 127.9, 45.9, 44.3, 36.6, 27.0, 25.2, 24.4; HRMS (EI) calcd for $\mathrm{C}_{13} \mathrm{H}_{16} \mathrm{OSe}\left(\mathrm{M}^{+}\right)$ 268.0361 , obsd 268.0343.<smiles>CC1(C)CCC([SeH]c2ccccc2)C1=O</smiles>

2-(Phenylselanyl)cyclohexanone ${ }^{8}$ : The title compound was prepared according to the general procedure in $80 \%$ yield. ${ }^{1} \mathrm{H}$ NMR $\left(500 \mathrm{MHz} \mathrm{CDCl}_{3}\right): \delta 7.55(\mathrm{dd}, 2 \mathrm{H}, J=6.5,1.5 \mathrm{~Hz}), 7.31-7.26$ $(\mathrm{m}, 3 \mathrm{H}), 3.91(\mathrm{t}, 1 \mathrm{H}, J=5.0 \mathrm{~Hz}), 2.95(\mathrm{~m}, 1 \mathrm{H}), 2.36-2.28(\mathrm{~m}, 1 \mathrm{H}), 2.27-2.15(\mathrm{~m}, 2 \mathrm{H}), 2.00-1.95$ $(\mathrm{m}, 1 \mathrm{H}), 1.89-1.69(\mathrm{~m}, 3 \mathrm{H}), ;{ }^{13} \mathrm{C} \mathrm{NMR}\left(125 \mathrm{MHz}, \mathrm{CDCl}_{3}\right): \delta 208.1,134.8,129.4,128.8,128.3$, 51.8, 38.7, 34.2, 27.1, 23.1; HRMS (EI) calcd for $\mathrm{C}_{12} \mathrm{H}_{14} \mathrm{OSe}\left(\mathrm{M}^{+}\right)$254.0204, obsd 254.0122.<smiles>O=C1CCCCC1Sc1ccccc1</smiles>

Tetrahydro-3-(phenylselanyl)pyran-4-one: The title compound was prepared according to the general procedure in $79 \%$ yield. ${ }^{1} \mathrm{H}$ NMR $\left(500 \mathrm{MHz}, \mathrm{CDCl}_{3}\right): \delta 7.54(\mathrm{dd}, 2 \mathrm{H}, J=9.5,2.0 \mathrm{~Hz})$, 7.33-7.26 (m, 3H), 4.16-4.03 (m, 3H), 3.96-3.89 (m, 2H), 3.14-3.09 (m, 1H), 2.51-2.47 (m, 1H); ${ }^{13} \mathrm{C} \mathrm{NMR}\left(125 \mathrm{MHz}, \mathrm{CDCl}_{3}\right): \delta 203.2,135.0,129.5,128.6,127.8,73.2,68.5,51.3,40.5$; HRMS (EI) calcd for $\mathrm{C}_{11} \mathrm{H}_{12} \mathrm{O}_{2} \mathrm{Se}\left(\mathrm{M}^{+}\right)$255.9997, obsd 255.9974.<smiles>O=C1CCOCC1Sc1ccccc1</smiles>

1-Methyl-3-(phenylselanyl)piperidin-4-one: The title compound was prepared according to the general procedure in $76 \%$ yield. ${ }^{1} \mathrm{H}$ NMR $\left(500 \mathrm{MHz}, \mathrm{CDCl}_{3}\right): \delta 7.54(\mathrm{dd}, 2 \mathrm{H}, J=9.0,2.0 \mathrm{~Hz})$, 7.29-7.25 (m, 3H), 3.76 (brs, $1 \mathrm{H}), 3.33-3.27(\mathrm{~m}, 1 \mathrm{H}), 3.17-3.14(\mathrm{~m}, 1 \mathrm{H}), 2.97-2.88(\mathrm{~m}, 2 \mathrm{H})$, 2.50-2.45 (m, 1H), $2.38(\mathrm{~s}, 3 \mathrm{H}), 2.37-2.32(\mathrm{~m}, 1 \mathrm{H}) ;{ }^{13} \mathrm{C} \mathrm{NMR}\left(125 \mathrm{MHz}, \mathrm{CDCl}_{3}\right): \delta 205.3$, 134.2, 129.5, 129.4, 128.1, 61.8, 55.7, 50.3, 45.9, 37.7; HRMS (EI) calcd for $\mathrm{C}_{12} \mathrm{H}_{15} \mathrm{NOSe}\left(\mathrm{M}^{+}\right)$ 269.0313, obsd 269.0341.<smiles>CN1CCC(=O)C(Sc2ccccc2)C1</smiles>

1-Ethylene ketal-3-(phenylselanyl)cyclohexanedin-4-one: ${ }^{9}$ The title compound was prepared 
according to the general procedure in $85 \%$ yield. ${ }^{1} \mathrm{H}$ NMR $\left(500 \mathrm{MHz}, \mathrm{CDCl}_{3}\right): \delta 7.54(\mathrm{dd}, 2 \mathrm{H}, J$ $=5.0,2.0 \mathrm{~Hz}), 7.28-7.25(\mathrm{~m}, 3 \mathrm{H}), 4.07-3.98(\mathrm{~m}, 5 \mathrm{H}), 3.18-3.12(\mathrm{~m}, 1 \mathrm{H}), 2.50-2.42(\mathrm{~m}, 2 \mathrm{H}), 2.32$ $(\mathrm{dd}, 1 \mathrm{H}, J=14.5,6.5 \mathrm{~Hz}), 2.04$ (t, $2 \mathrm{H}, J=7.0 \mathrm{~Hz}) ;{ }^{13} \mathrm{C} \mathrm{NMR}\left(125 \mathrm{MHz}, \mathrm{CDCl}_{3}\right): \delta 206.4,134.5$, 129.8, 129.3, 128.2, 107.0, 65.1, 64.9, 48.0, 41.0, 35.3, 34.6; HRMS (EI) calcd for $\mathrm{C}_{14} \mathrm{H}_{16} \mathrm{O}_{3} \mathrm{Se}$ $\left(\mathrm{M}^{+}\right)$312.0259, obsd 312.0260.<smiles>O=C1CCC2(CC1[Se]c1ccccc1)OCCO2</smiles>

6-(Phenylselanyl)cyclohex-2-enone: ${ }^{10}$ The title compound was prepared according to the general procedure in $63 \%$ yield. ${ }^{1} \mathrm{H}$ NMR $\left(500 \mathrm{MHz}, \mathrm{CDCl}_{3}\right): \delta 7.60(\mathrm{~d}, 2 \mathrm{H}, J=7.0 \mathrm{~Hz}), 7.33-$ $7.26(\mathrm{~m}, 3 \mathrm{H}), 6.93-6.90(\mathrm{~m}, 1 \mathrm{H}), 6.03(\mathrm{~d}, 1 \mathrm{H}, J=10.0 \mathrm{~Hz}), 4.03(\mathrm{t}, 1 \mathrm{H}, J=5.0 \mathrm{~Hz}), 2.60-2.53$ (m, 1H), 2.40-2.16 (m, 3H); ${ }^{13} \mathrm{C}$ NMR $\left(125 \mathrm{MHz}, \mathrm{CDCl}_{3}\right): \delta$ 195.3, 149.4, 135.6, 129.3, 128.6, 128.5, 127.8, 48.0, 29.3, 24.0; HRMS (EI) calcd for $\mathrm{C}_{12} \mathrm{H}_{12} \mathrm{OSe}\left(\mathrm{M}^{+}\right)$252.0048, obsd 252.0043.<smiles>O=C1C=CCCC1Sc1ccccc1</smiles>

2-(Phenylselanyl)cycloheptanone: ${ }^{9}$ The title compound was prepared according to the general procedure in $59 \%$ yield. ${ }^{1} \mathrm{H}$ NMR $\left(500 \mathrm{MHz}, \mathrm{CDCl}_{3}\right): \delta 7.54(\mathrm{dd}, 2 \mathrm{H}, J=6.0,2.0 \mathrm{~Hz}), 7.32-7.26$ $(\mathrm{m}, 3 \mathrm{H}), 3.80(\mathrm{dd}, 1 \mathrm{H}, J=11.0,5.5 \mathrm{~Hz}), 2.77-2.71(\mathrm{~m}, 1 \mathrm{H}), 2.41-2.37(\mathrm{~m}, 1 \mathrm{H}), 2.32-2.26(\mathrm{~m}$, $1 \mathrm{H}), 1.95-1.87(\mathrm{~m}, 2 \mathrm{H}), 1.85-1.80(\mathrm{~m}, 1 \mathrm{H}), 1.67-1.60(\mathrm{~m}, 1 \mathrm{H}), 1.51-1.35(\mathrm{~m}, 2 \mathrm{H}), 1.33-1.21(\mathrm{~m}$, $1 \mathrm{H}) ;{ }^{13} \mathrm{C}$ NMR $\left(125 \mathrm{MHz}, \mathrm{CDCl}_{3}\right): \delta 209.5,135.2,129.3,128.6,128.5,52.5,40.1,30.7,30.4$, 28.2, 25.9; HRMS (EI) calcd for $\mathrm{C}_{13} \mathrm{H}_{16} \mathrm{OSe}\left(\mathrm{M}^{+}\right)$268.0361, obsd 268.0342.

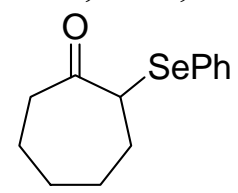

\section{References}

(1) Known compounds, reported in: Houllemare, D.; Ponthieux, S.; Outurquin, F.; Paulmier, C. Synthesis 1997, 101.

(2) Known compound, reported in: Shea, R. G.; Fitzner, J. N.; Fankhauser, J. E.; Spaltenstein, A.; Carpino, P. A.; Peevey, R. M.; Pratt, D. V.; Tenge, B. J.; Hopkins, P. B. J. Org. Chem 1986, 51,5243

(3) Known compound, reported in: Di Giamberardino, T.; Halazy, S.; Dumont, W.; Krief, A. Tetrahedron Lett. 1983, 24, 3413.

(4) Known compound, reported in: Kuwajima, I.; Shimizu, M. Tetrahedron Lett. 1978, 19, 1277.

(5) Known compounds, reported in: Paulmier, C.; Lerouge, P. Tetrahedron Lett. 1982, 23, 1557.

(6) Known compound, reported in: Reich, H. J.; Renga, J. M.; Reich, I. L. J. Am. Chem. Soc. 1975, 97, 5434. 
(7) Known compound, reported in: Toru, T.; Kawai, S.; Ueno, Y. Synlett 1996, 539.

(8) Known compound, reported in: Ponthieux, S.; Outurquin, F.; Paulmier, C. Tetrahedron 1997, 53, 6365 .

(9) Known compound, reported in: Magnus, P.; Rigollier, P. Tetrahedron Lett. 1992, 33, 6111.

(10) Known compound, reported in: Cossy, J.; Furet, N. Tetrahedron Lett. 1993, 34, 7755. 


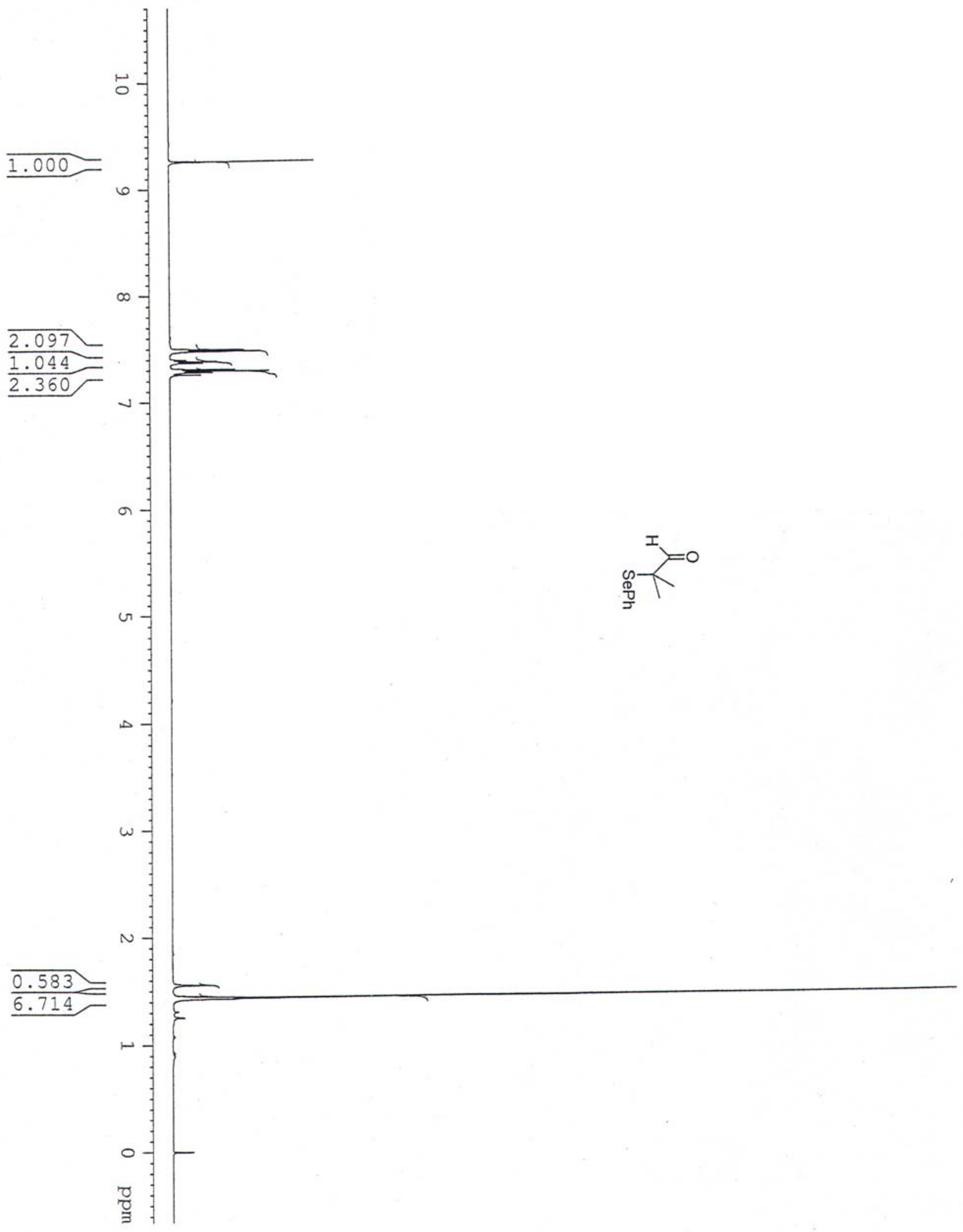




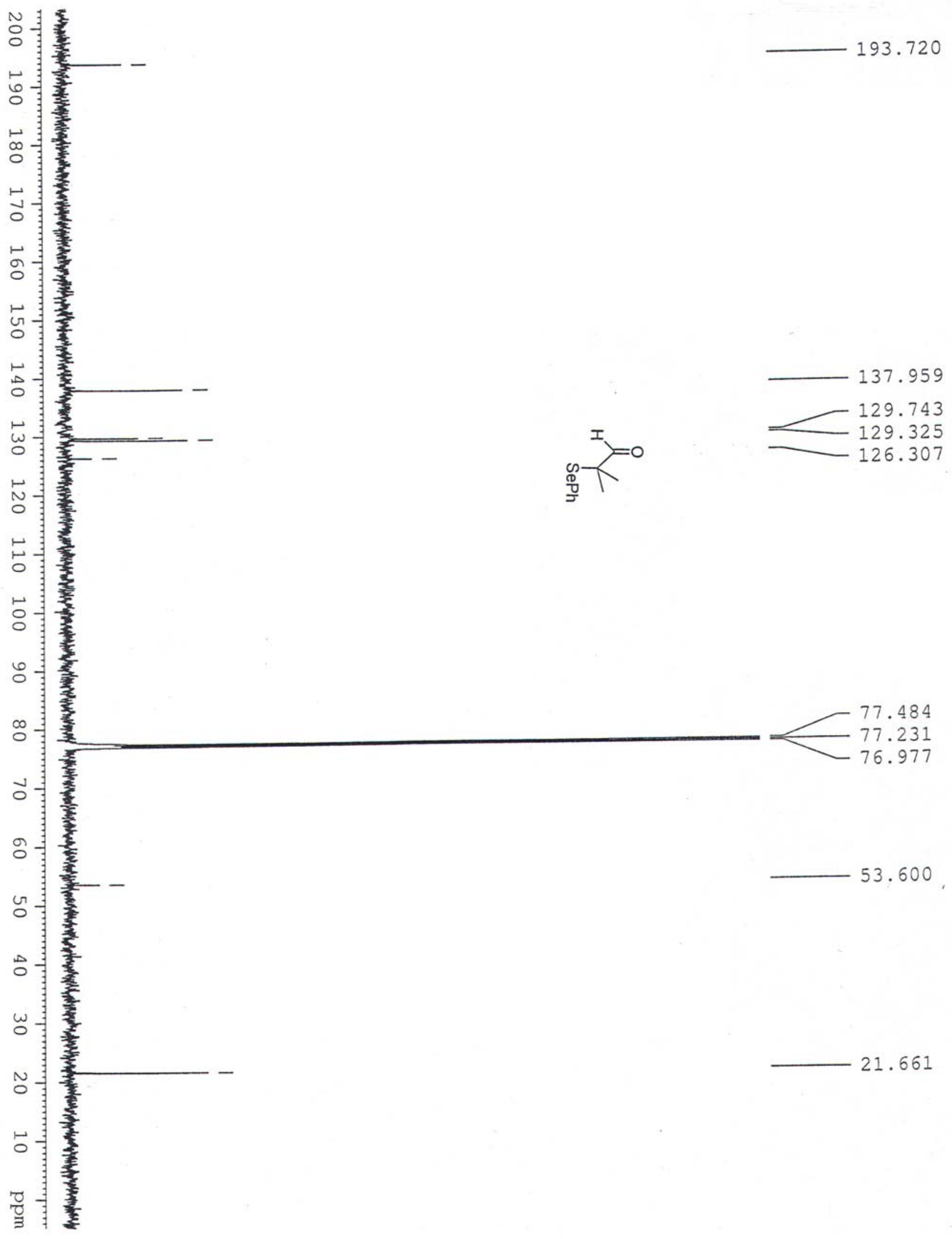




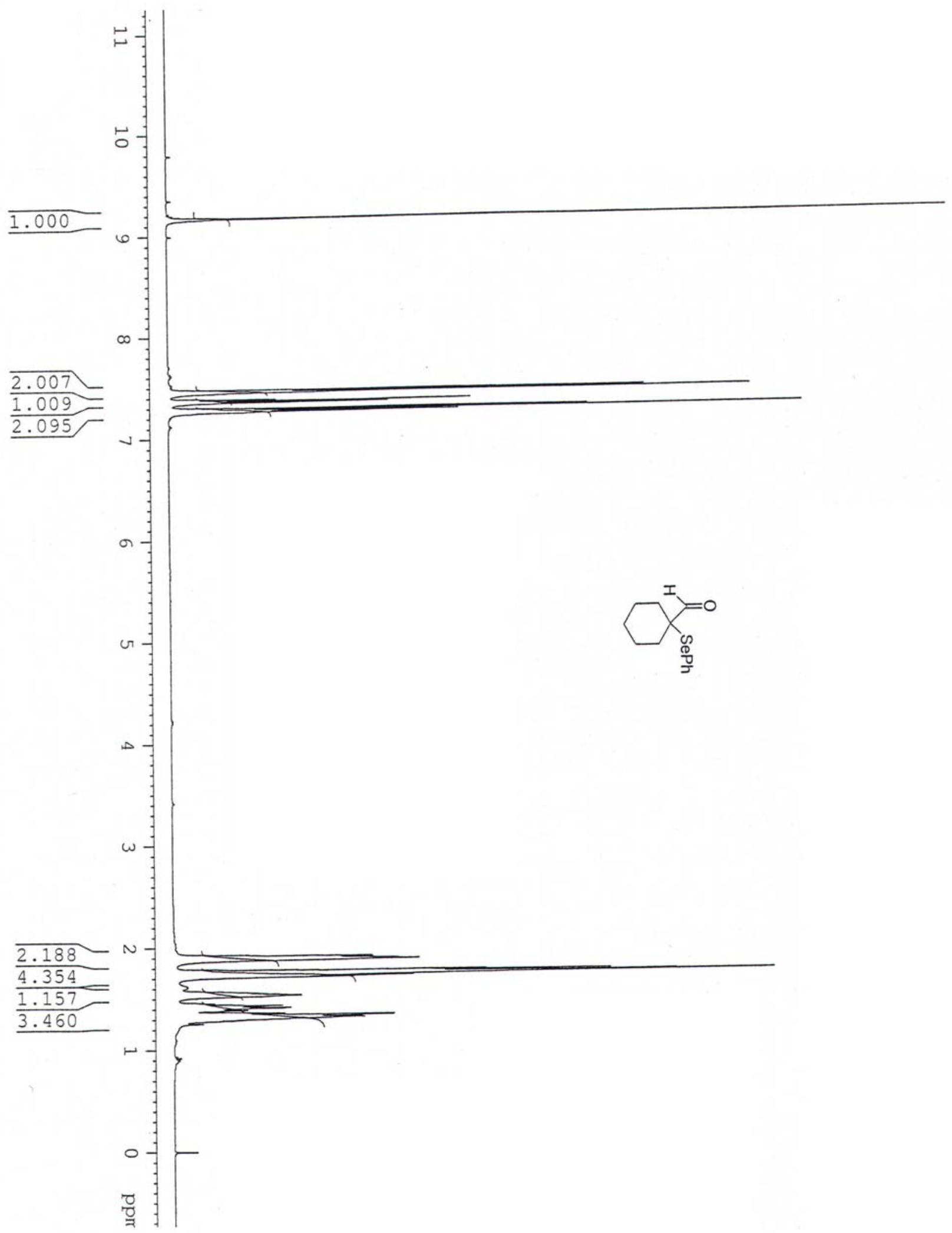




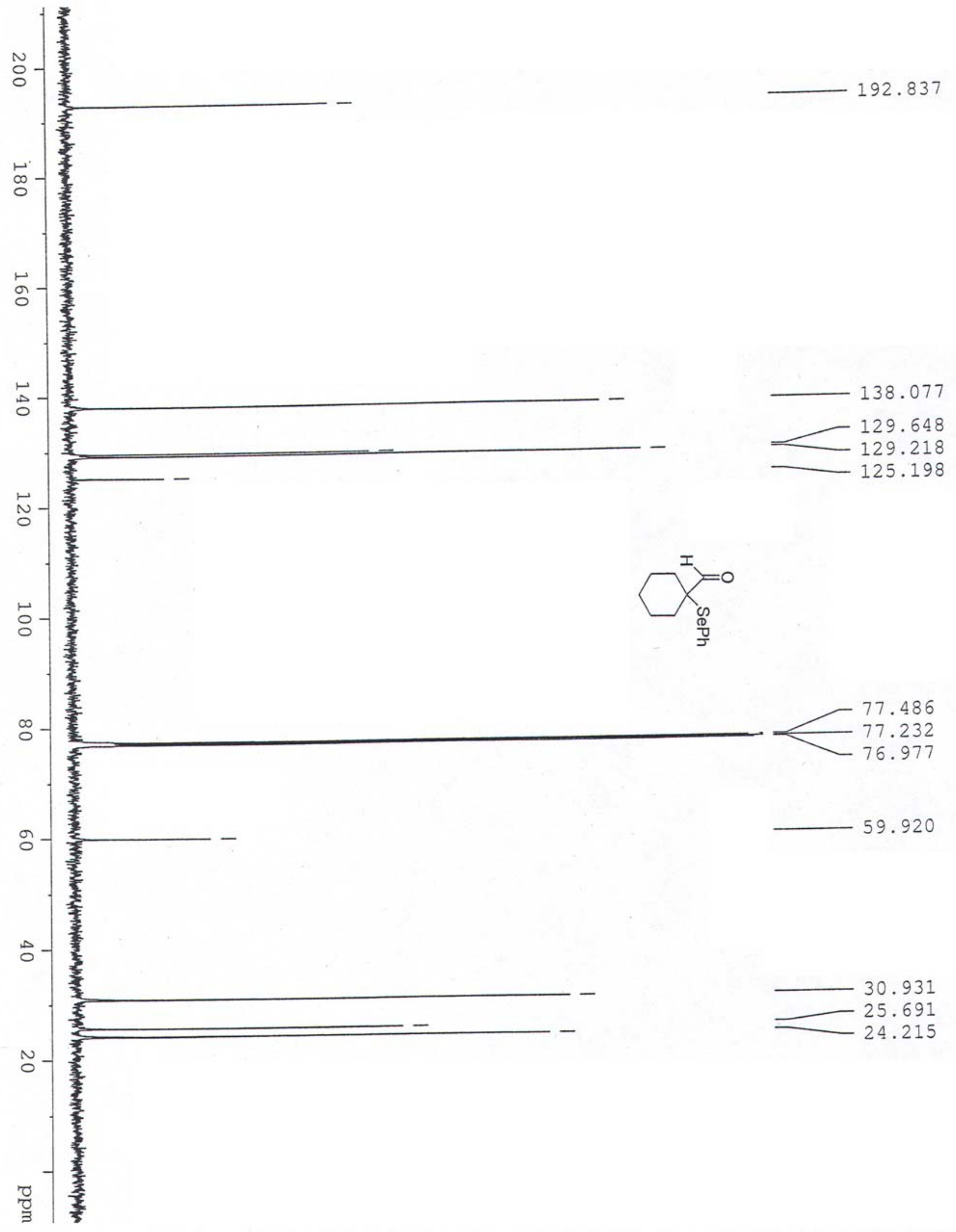




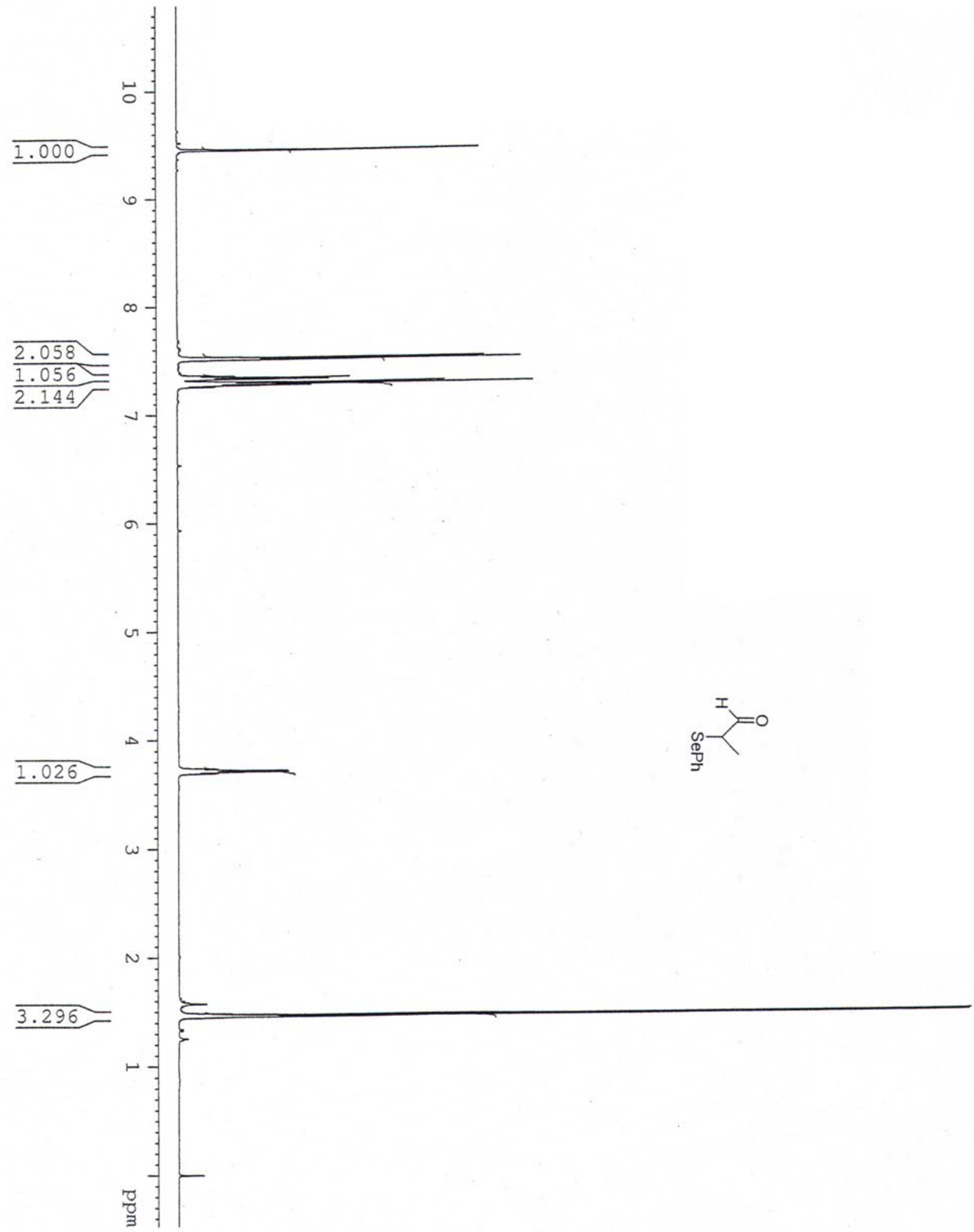




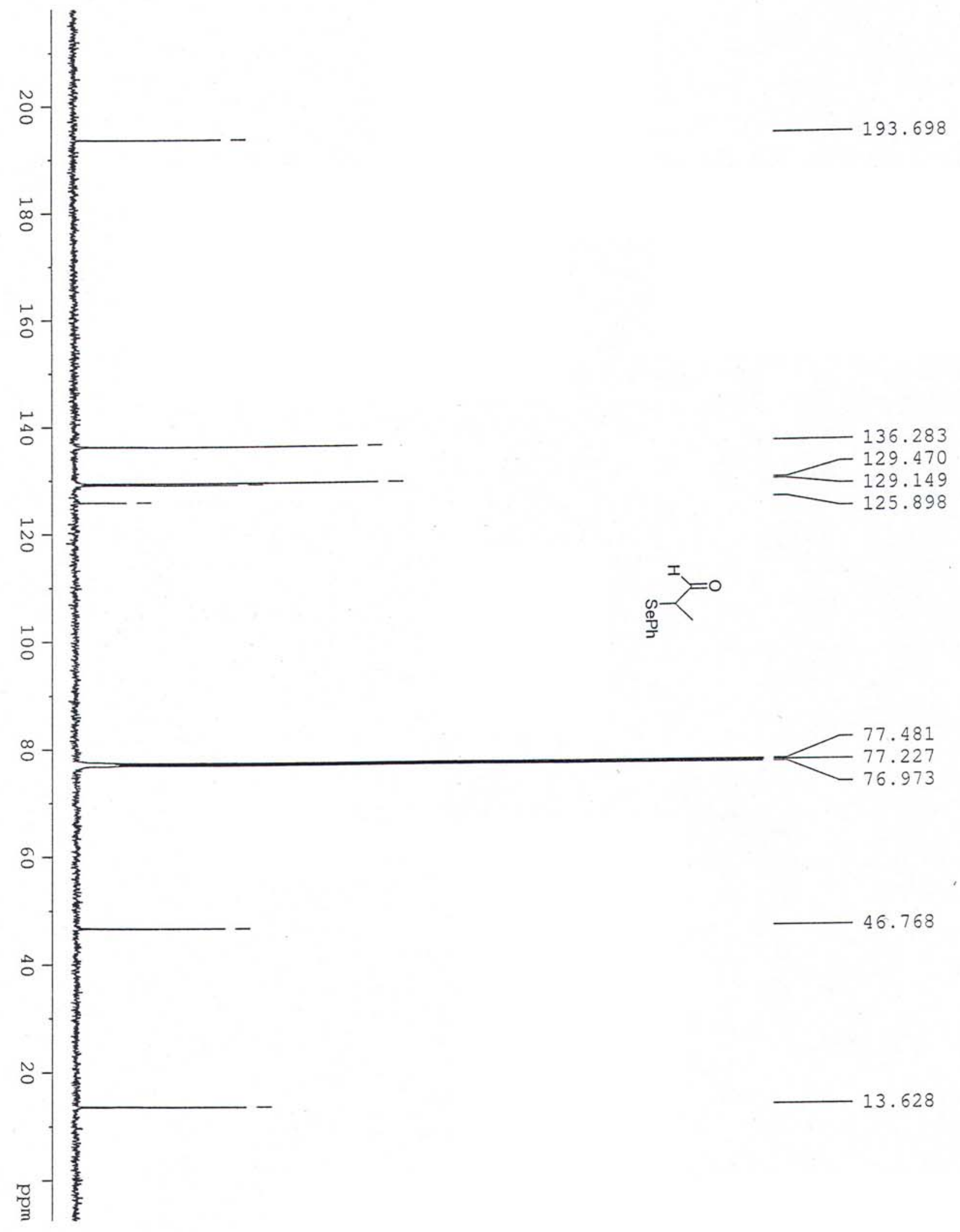




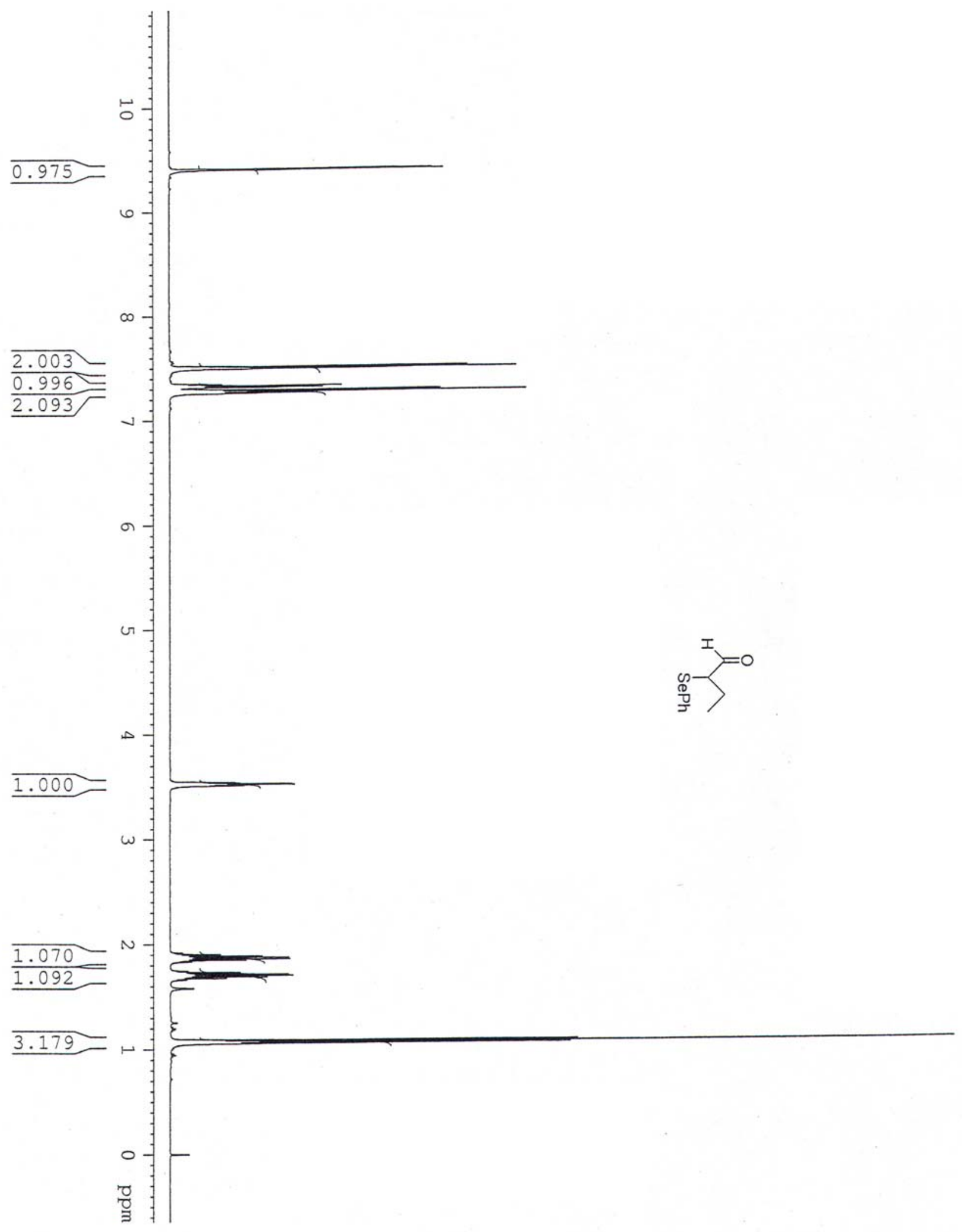

S16 


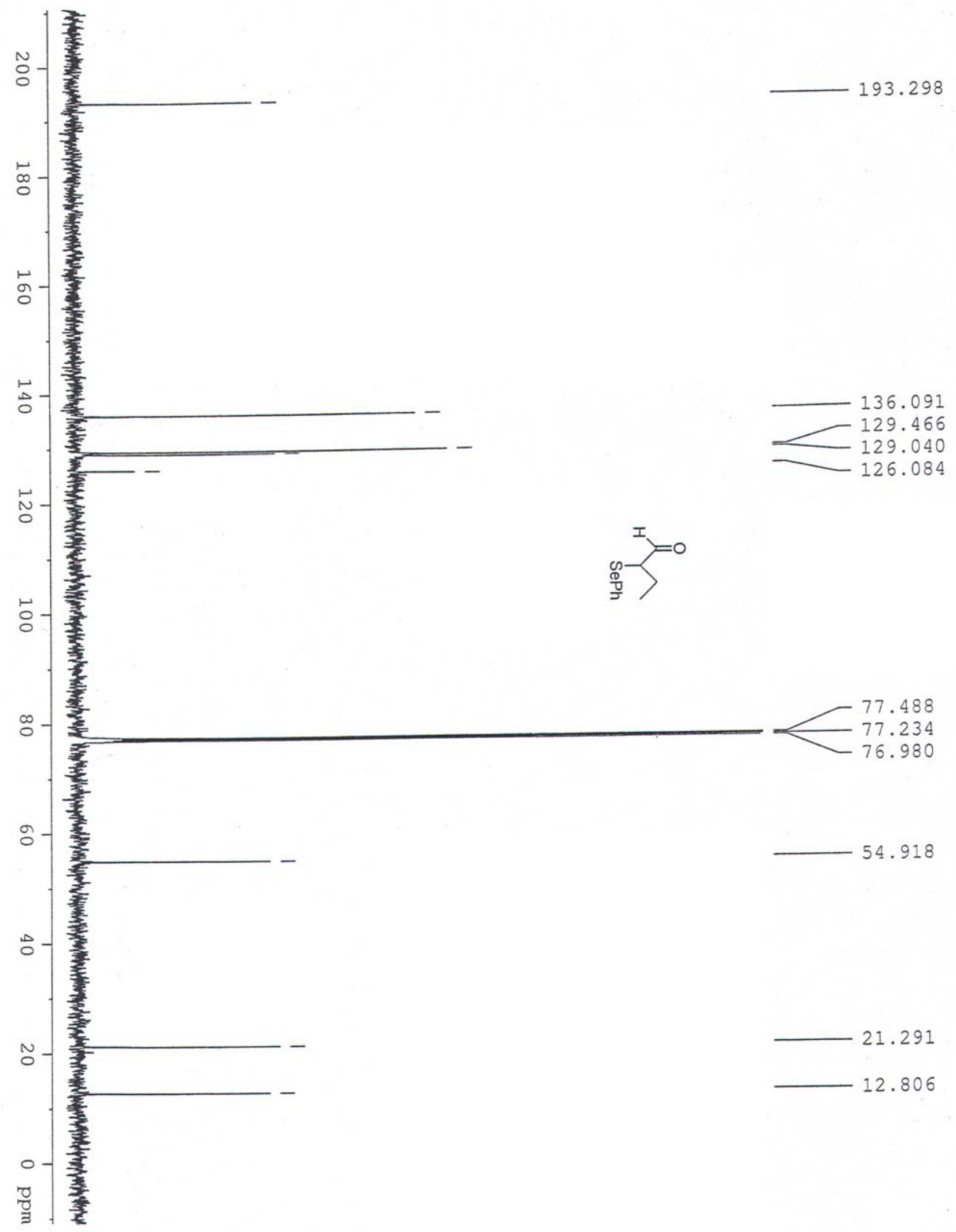




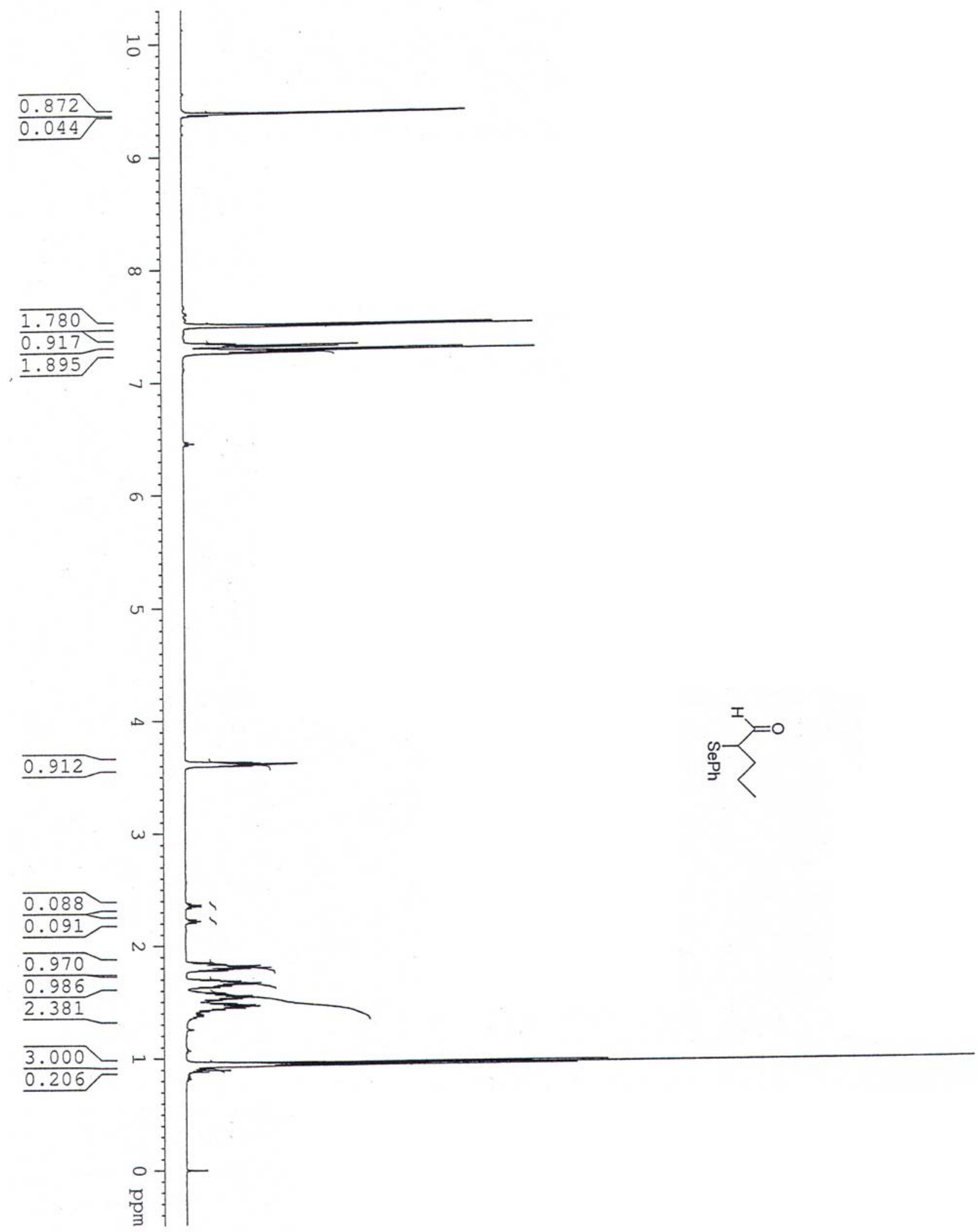




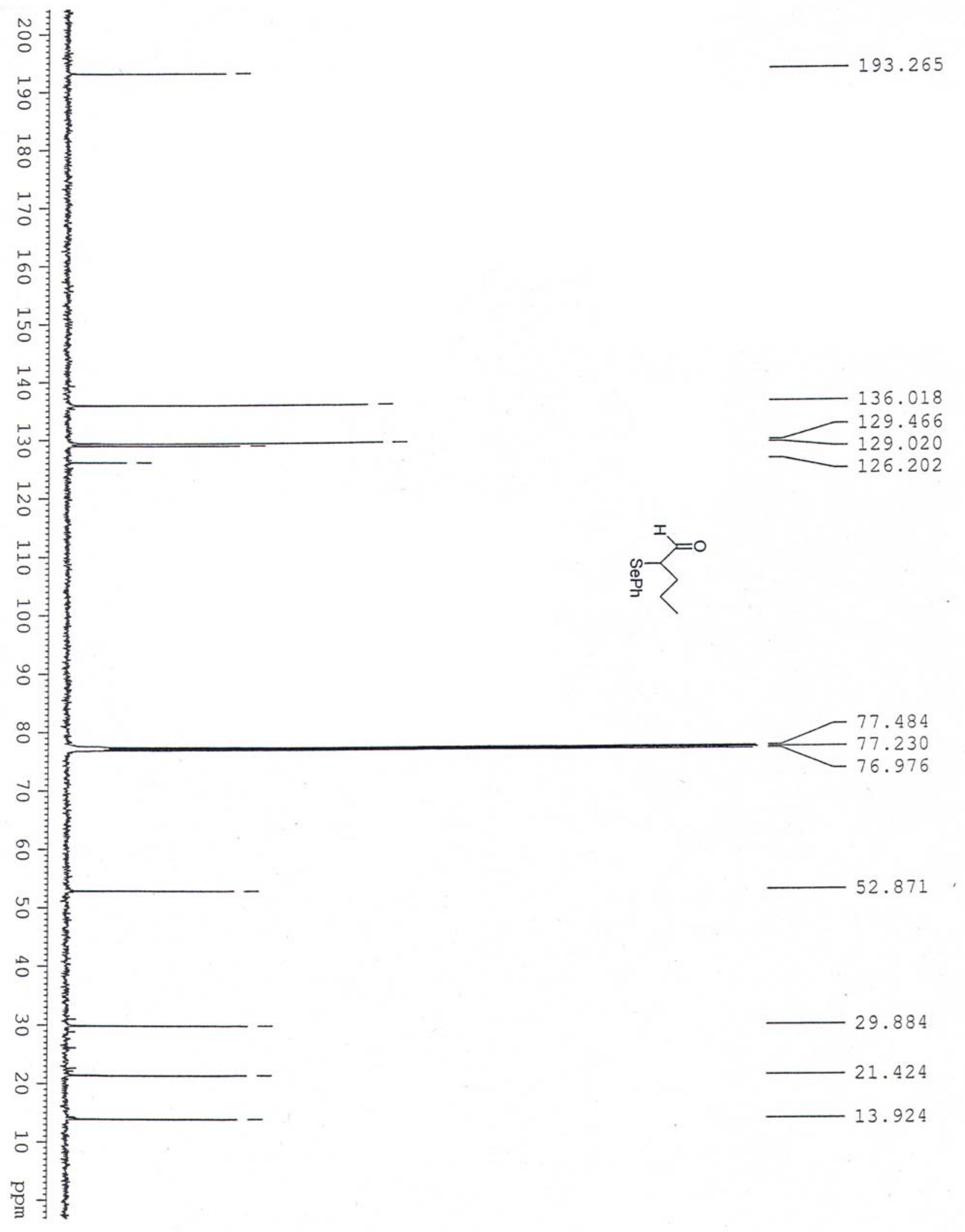




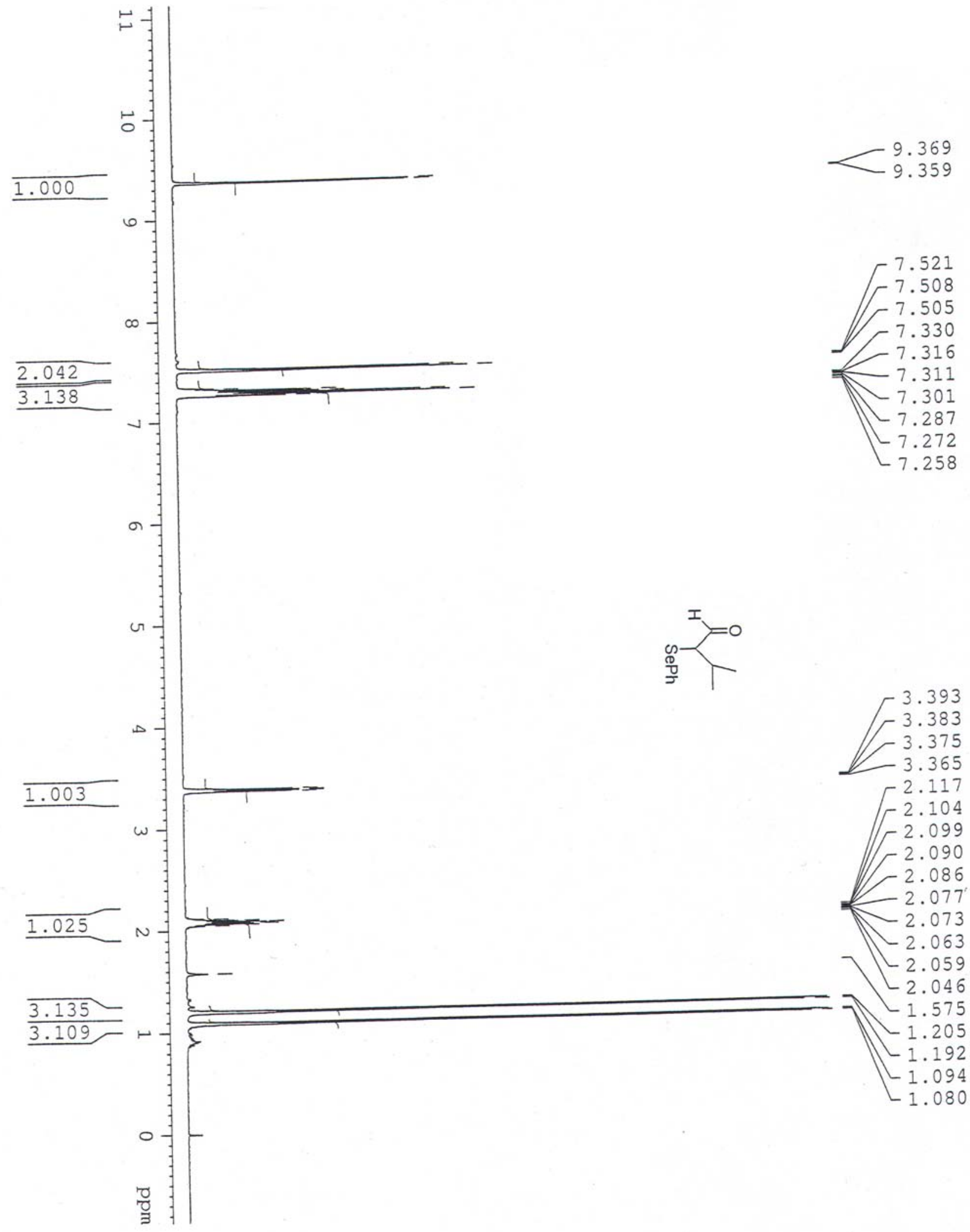




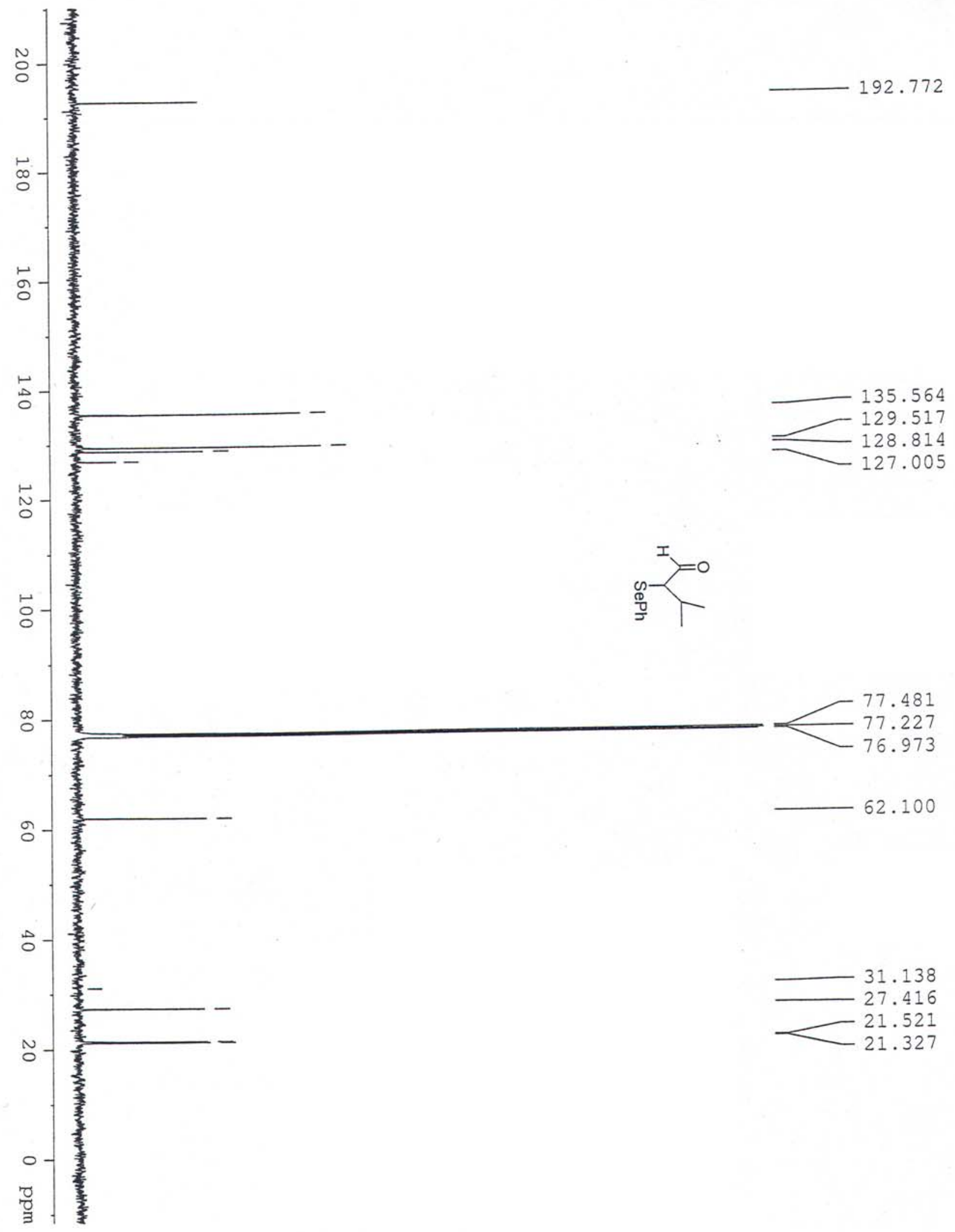




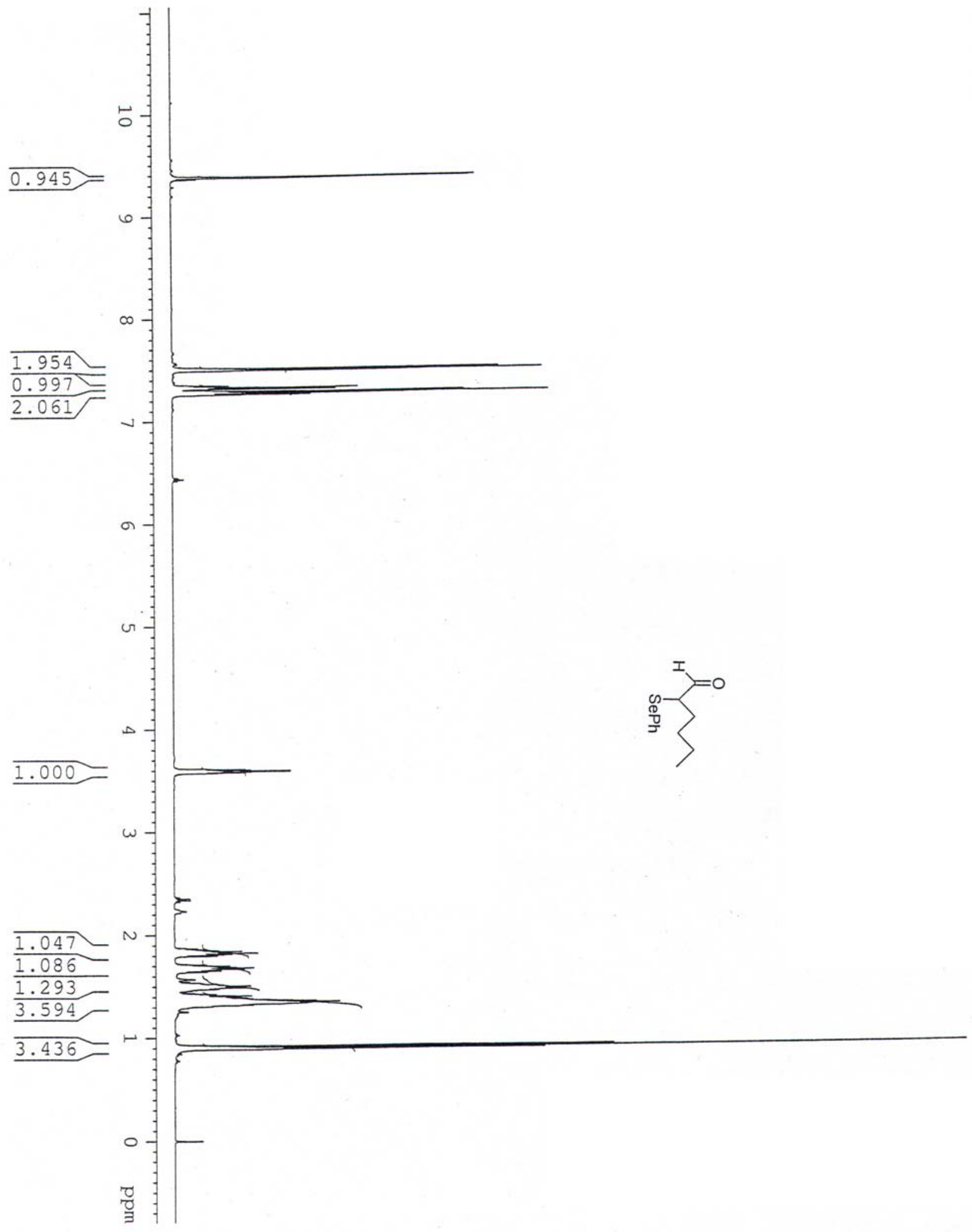




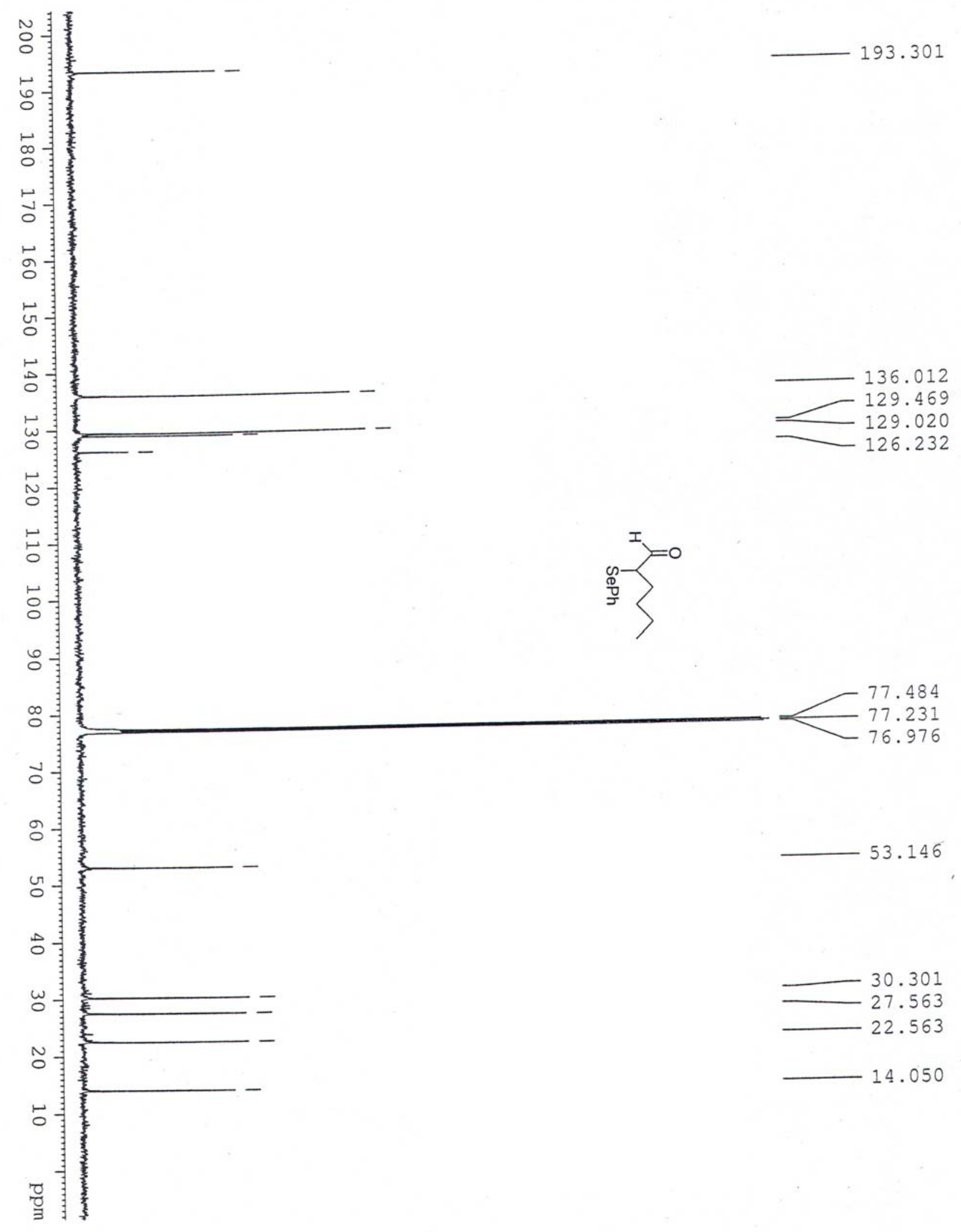




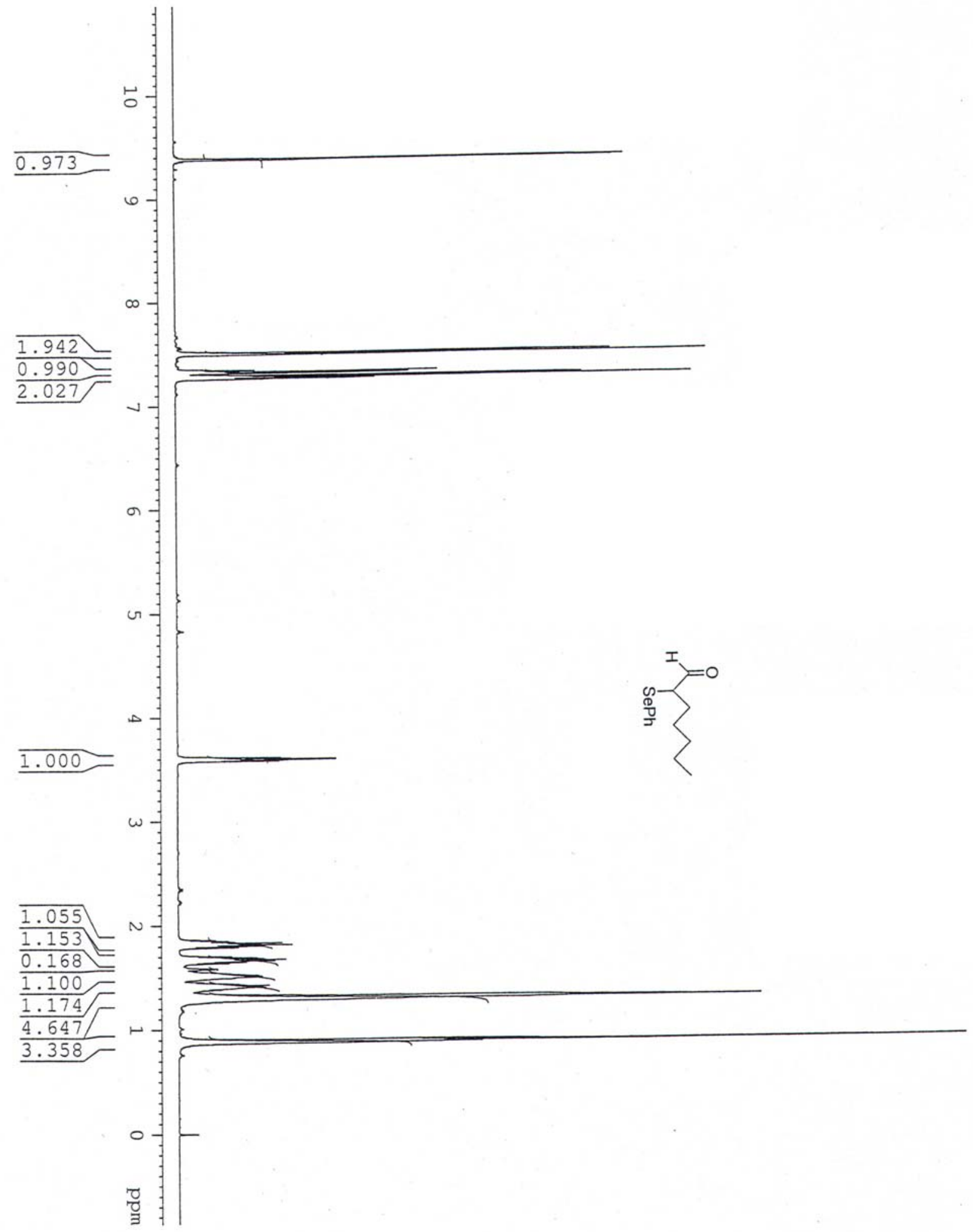




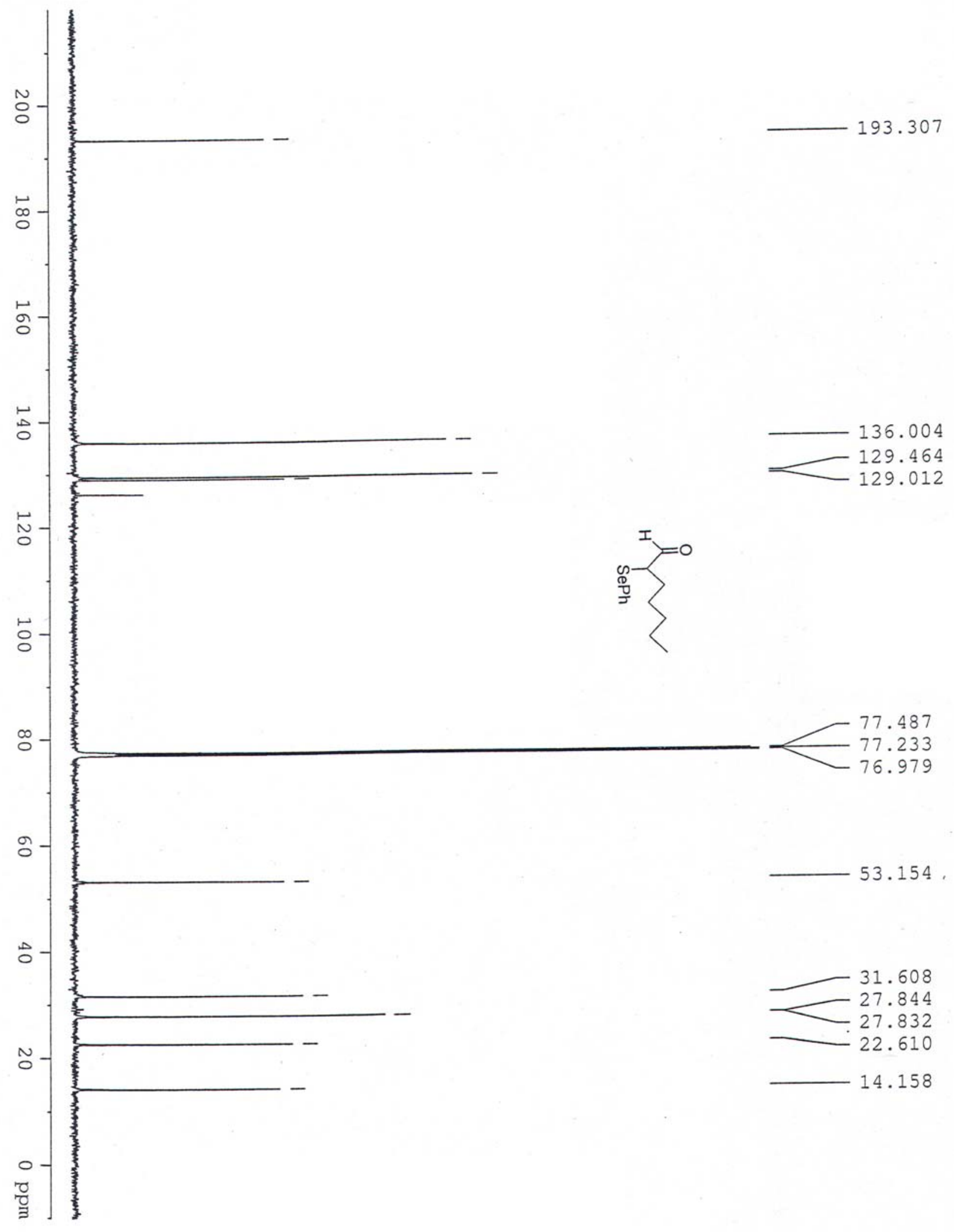




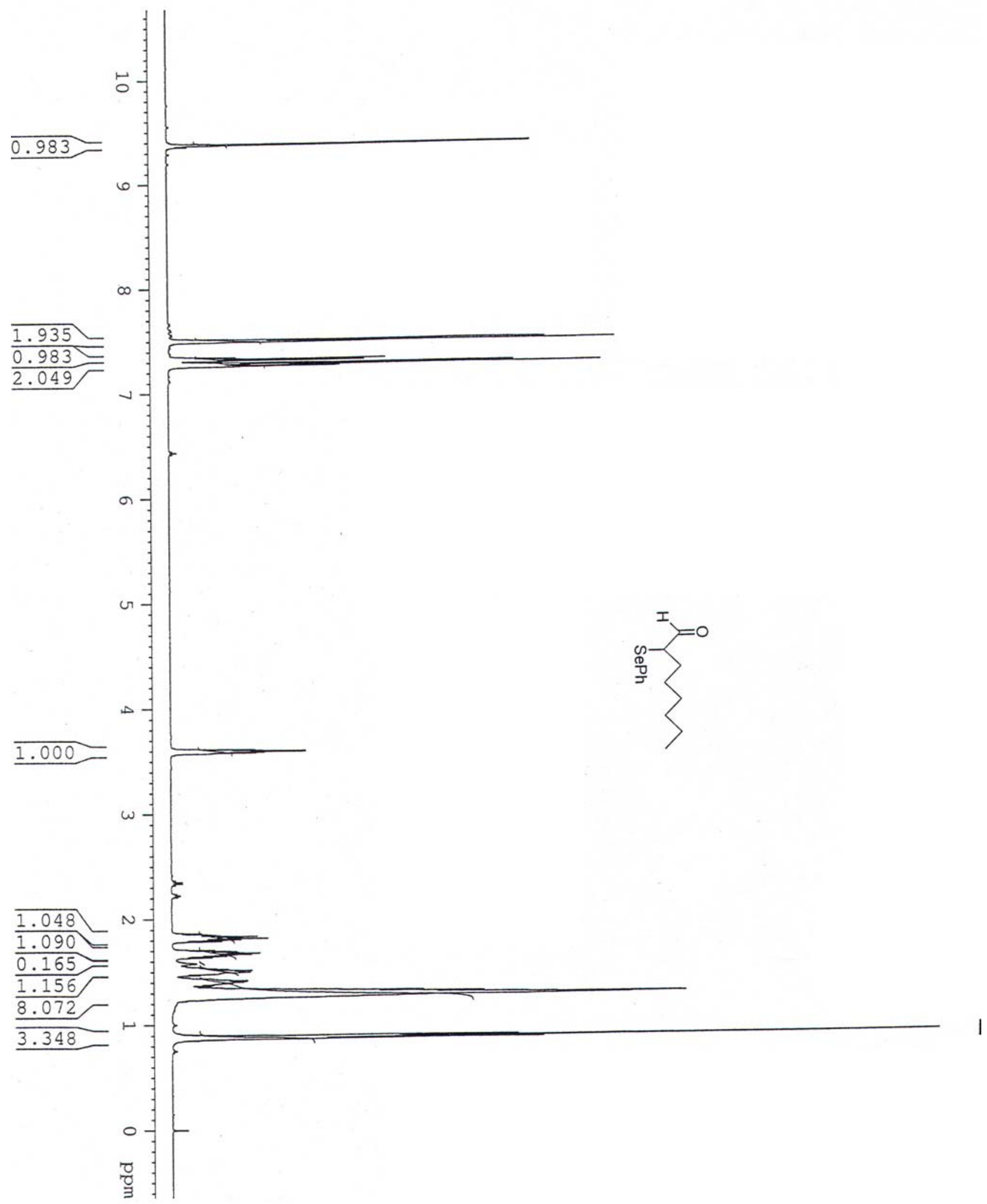




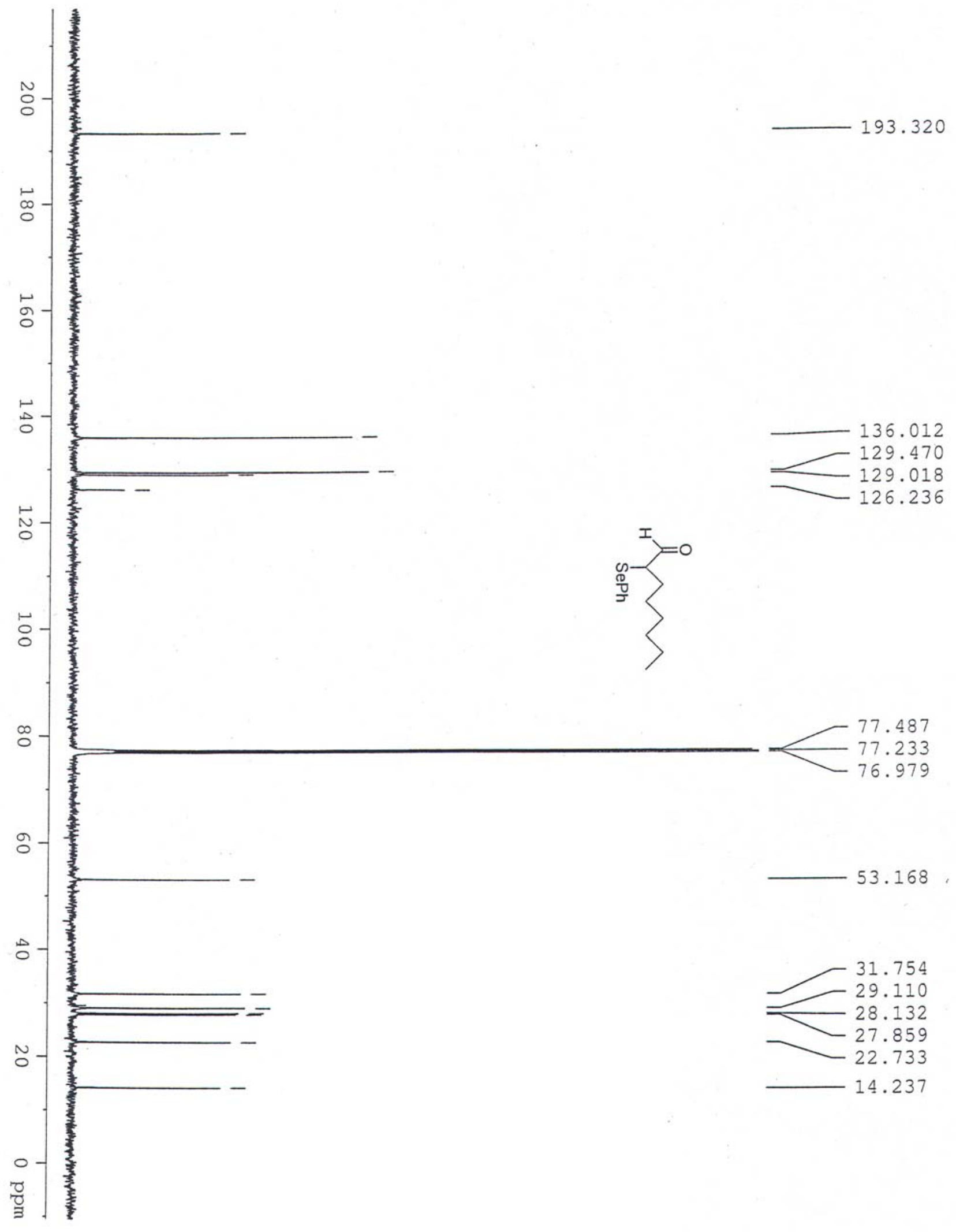




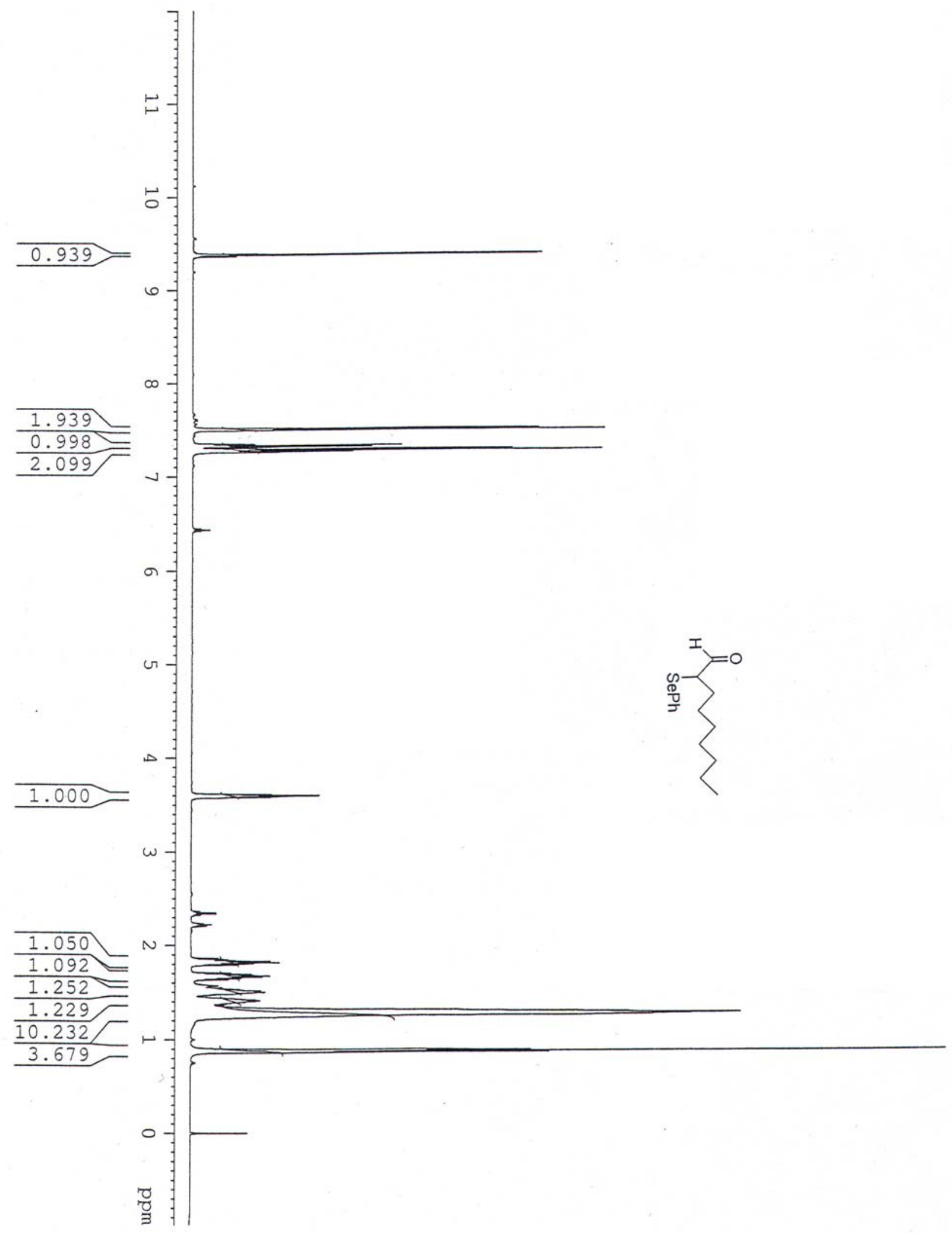




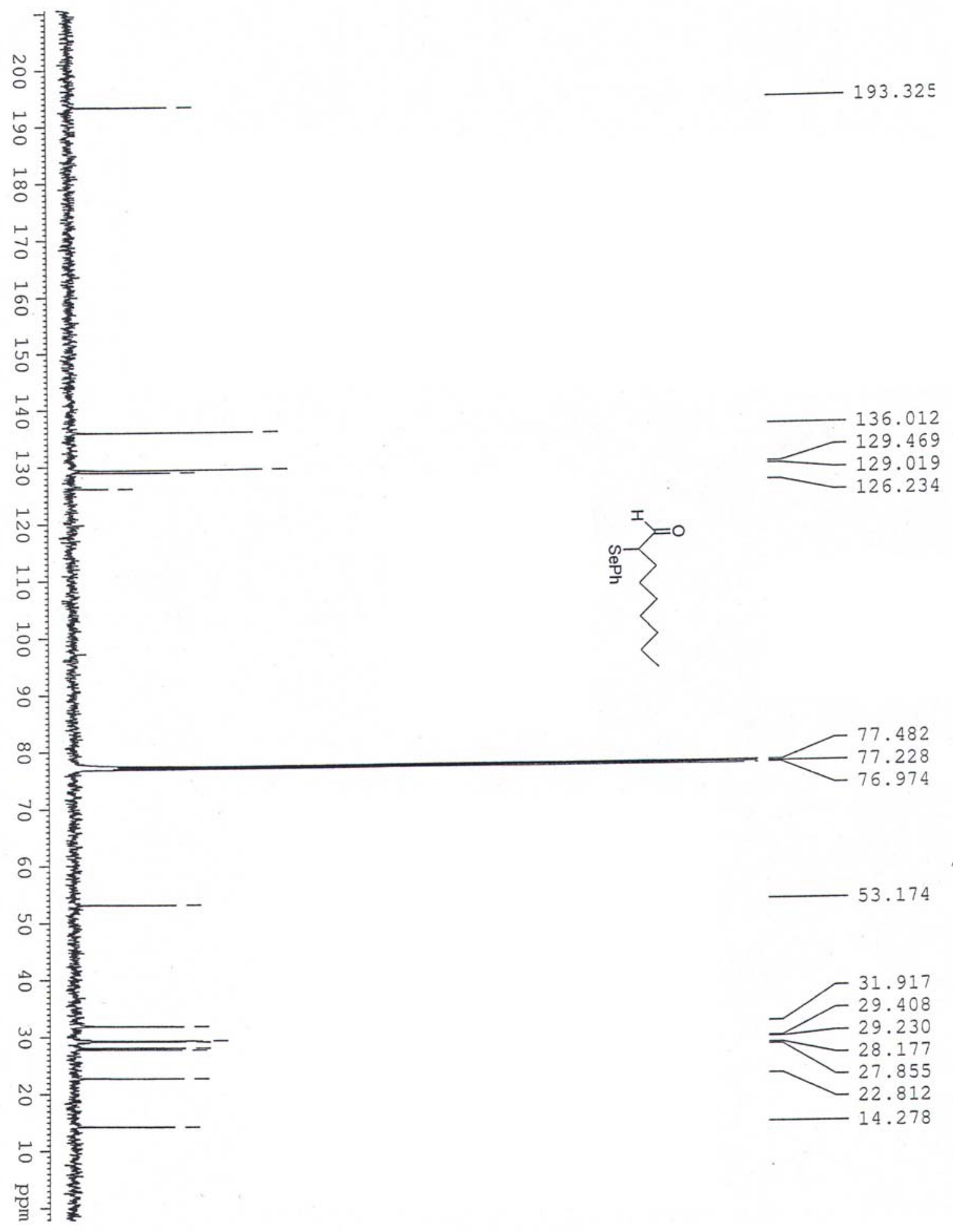




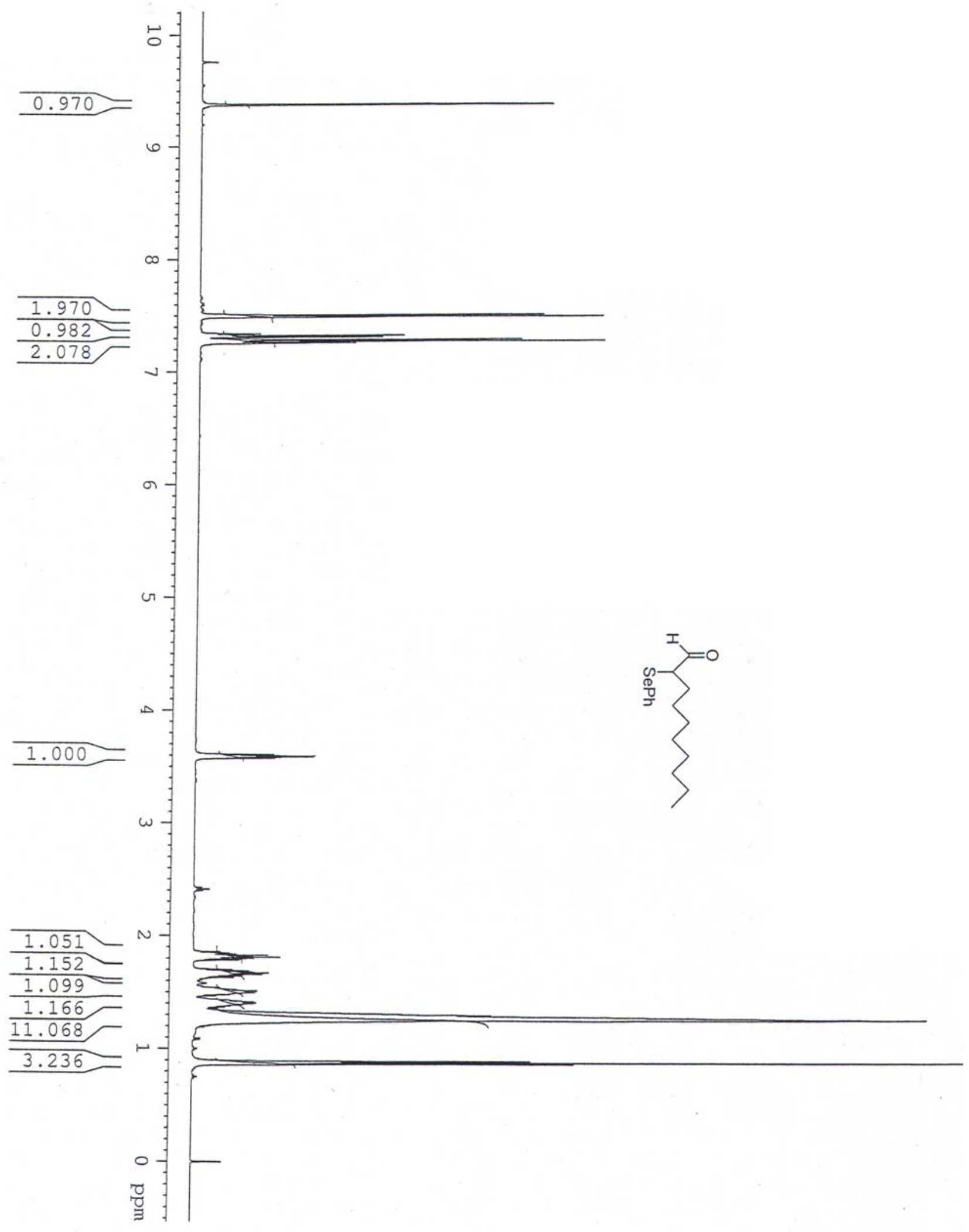




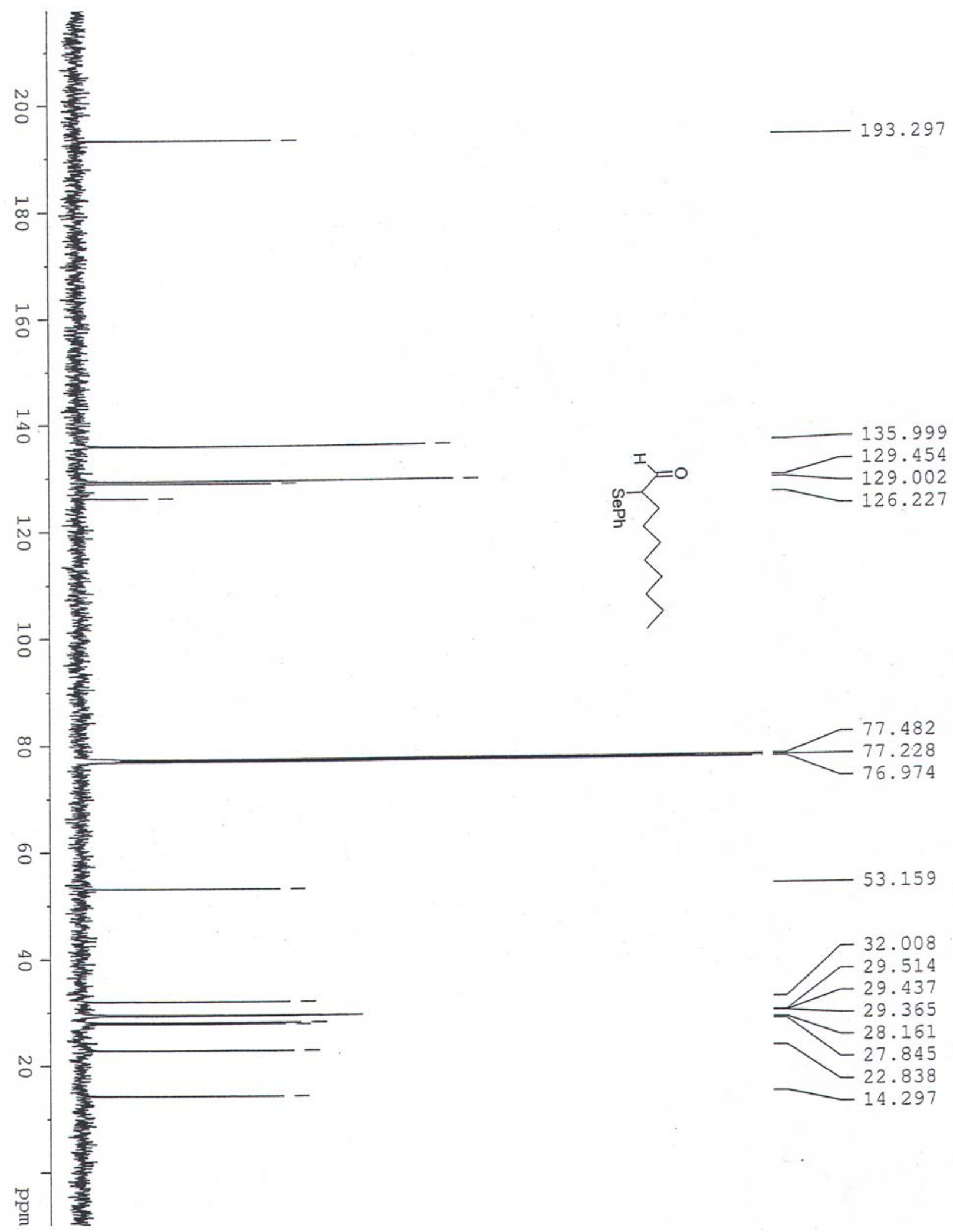




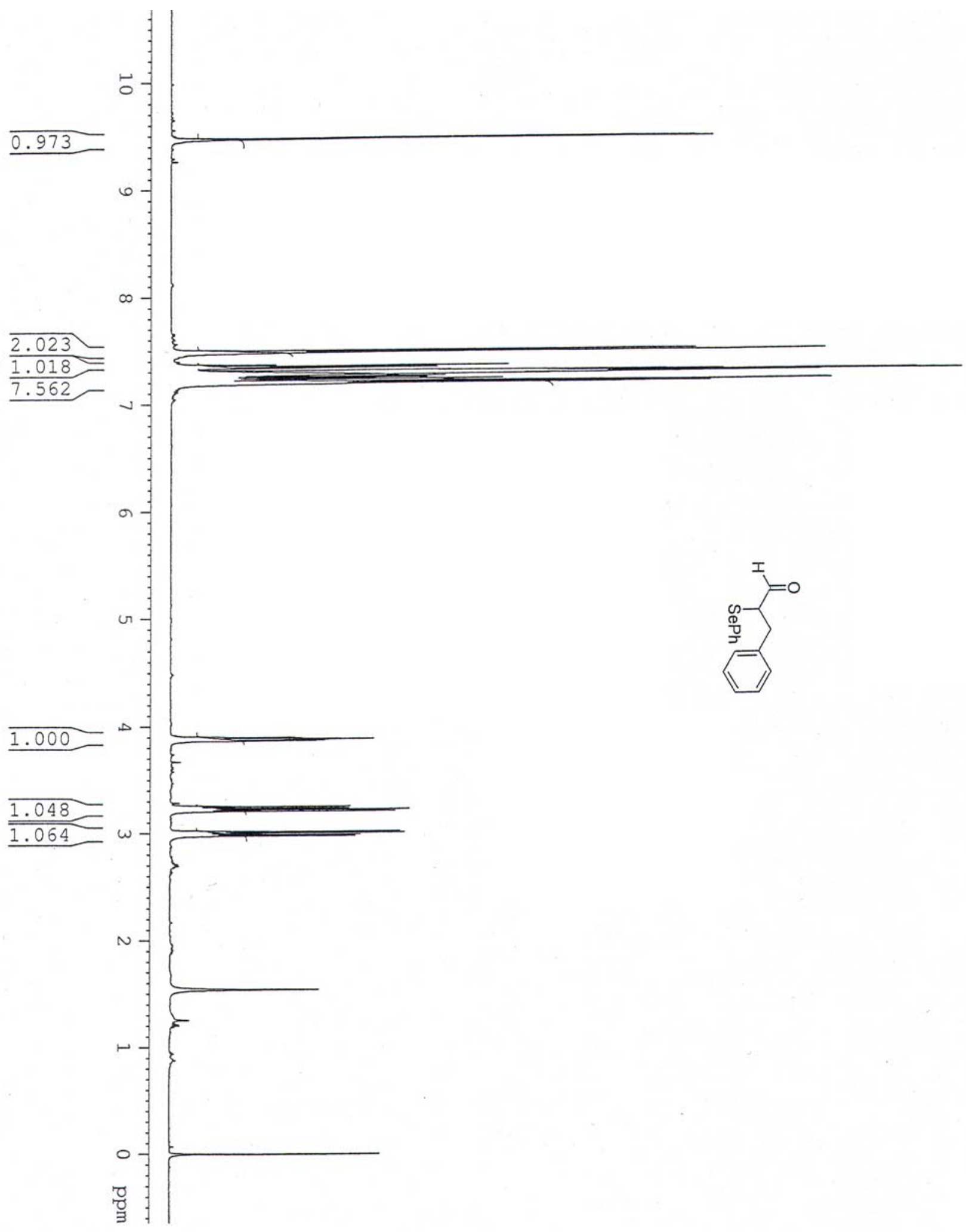




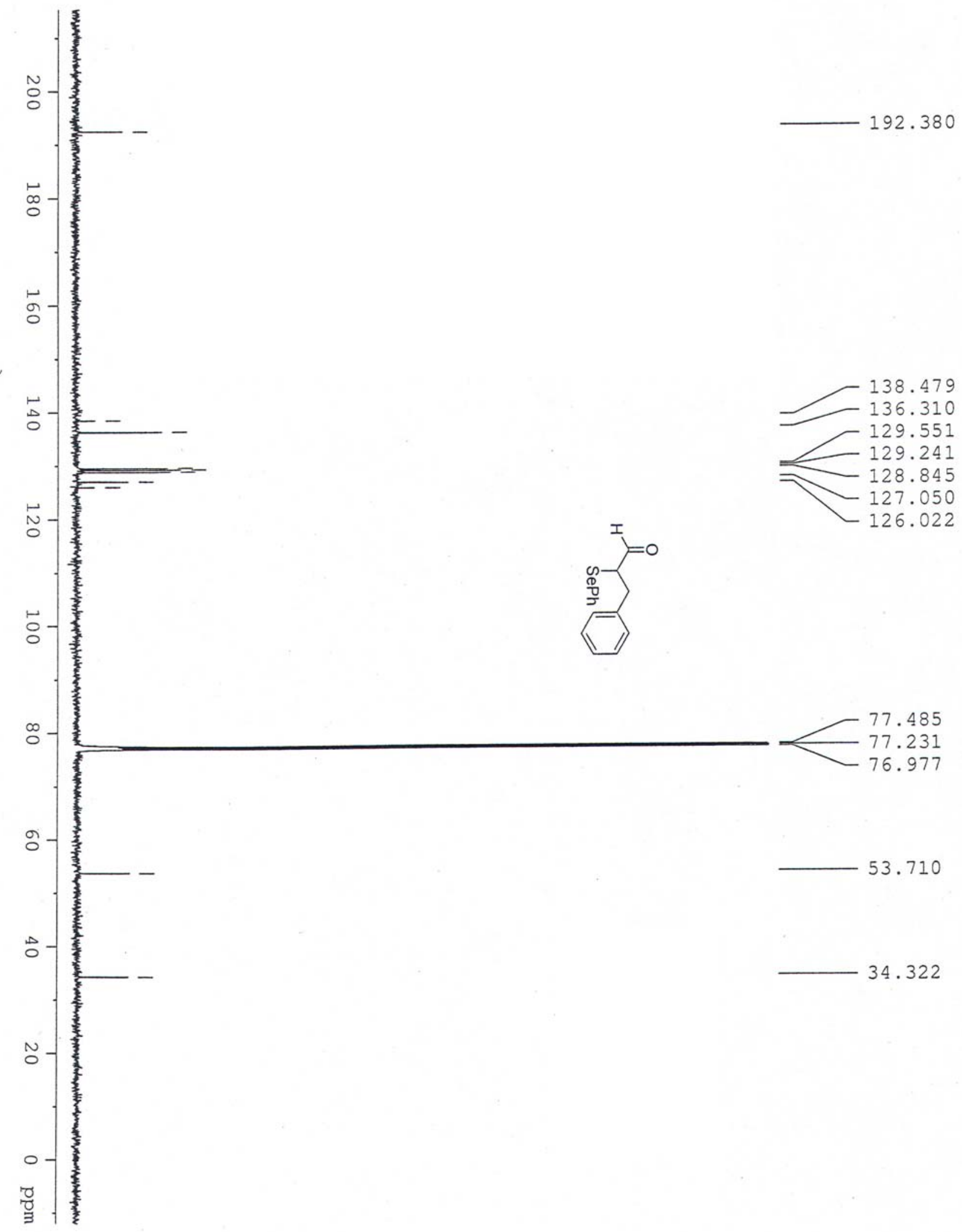




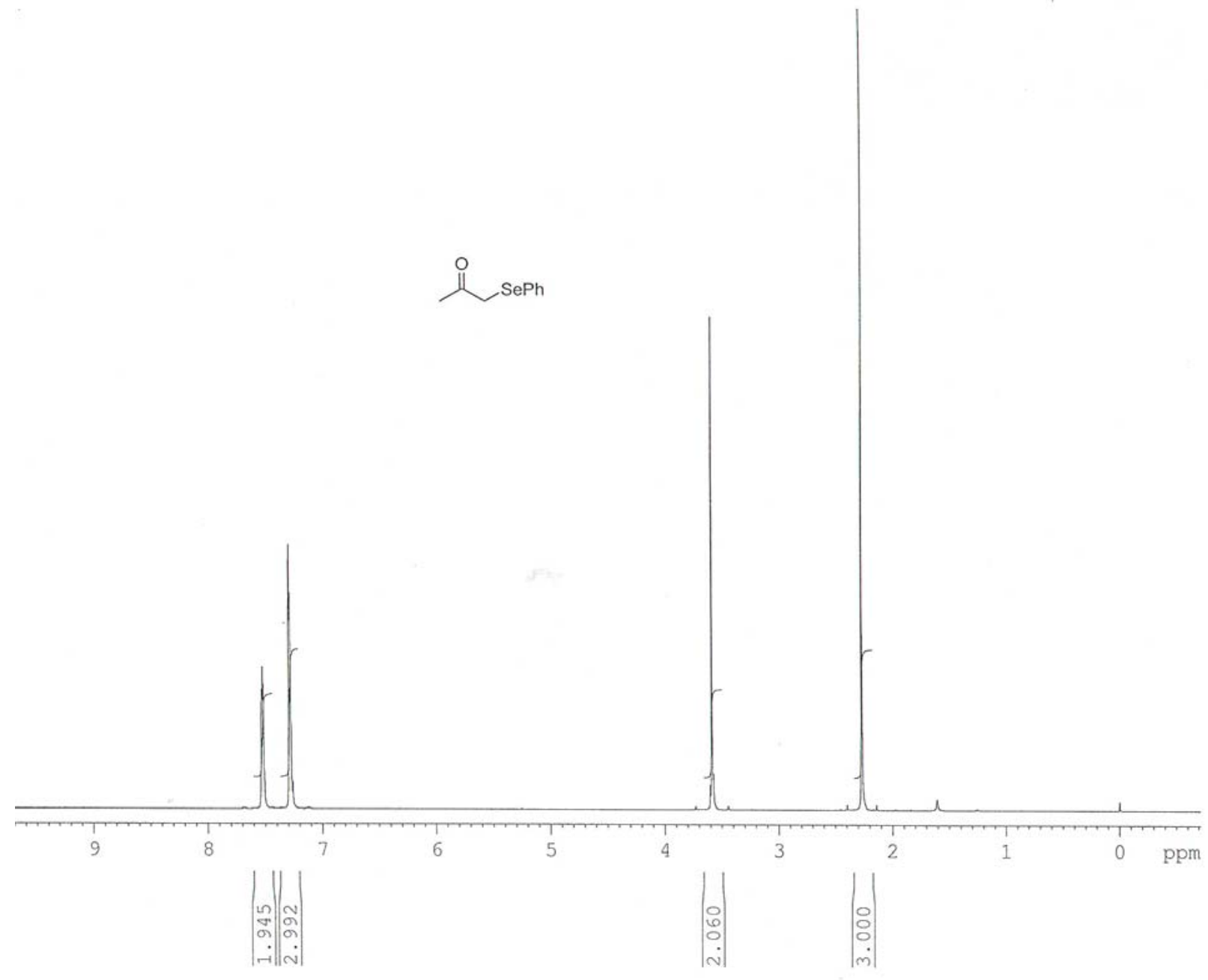




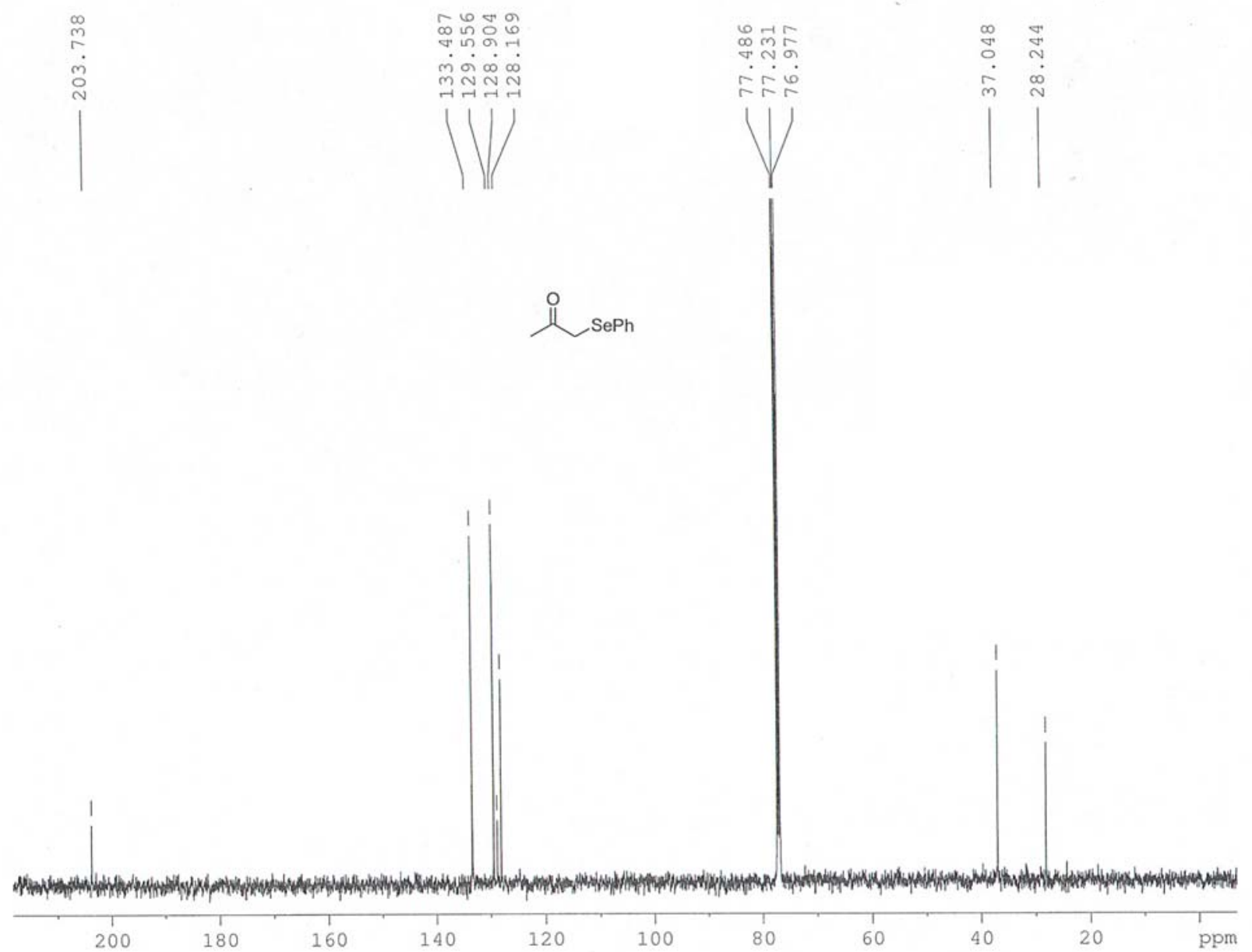




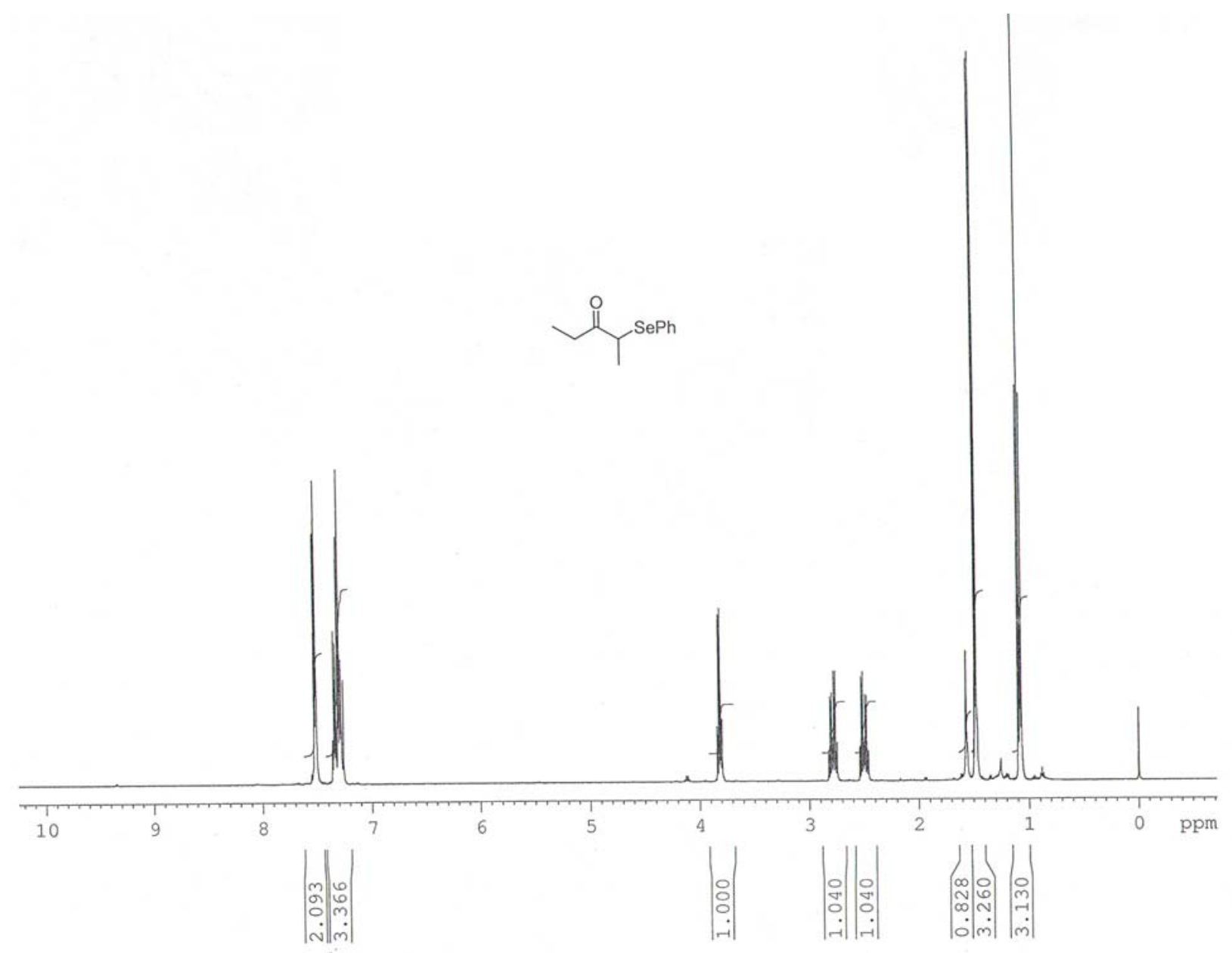




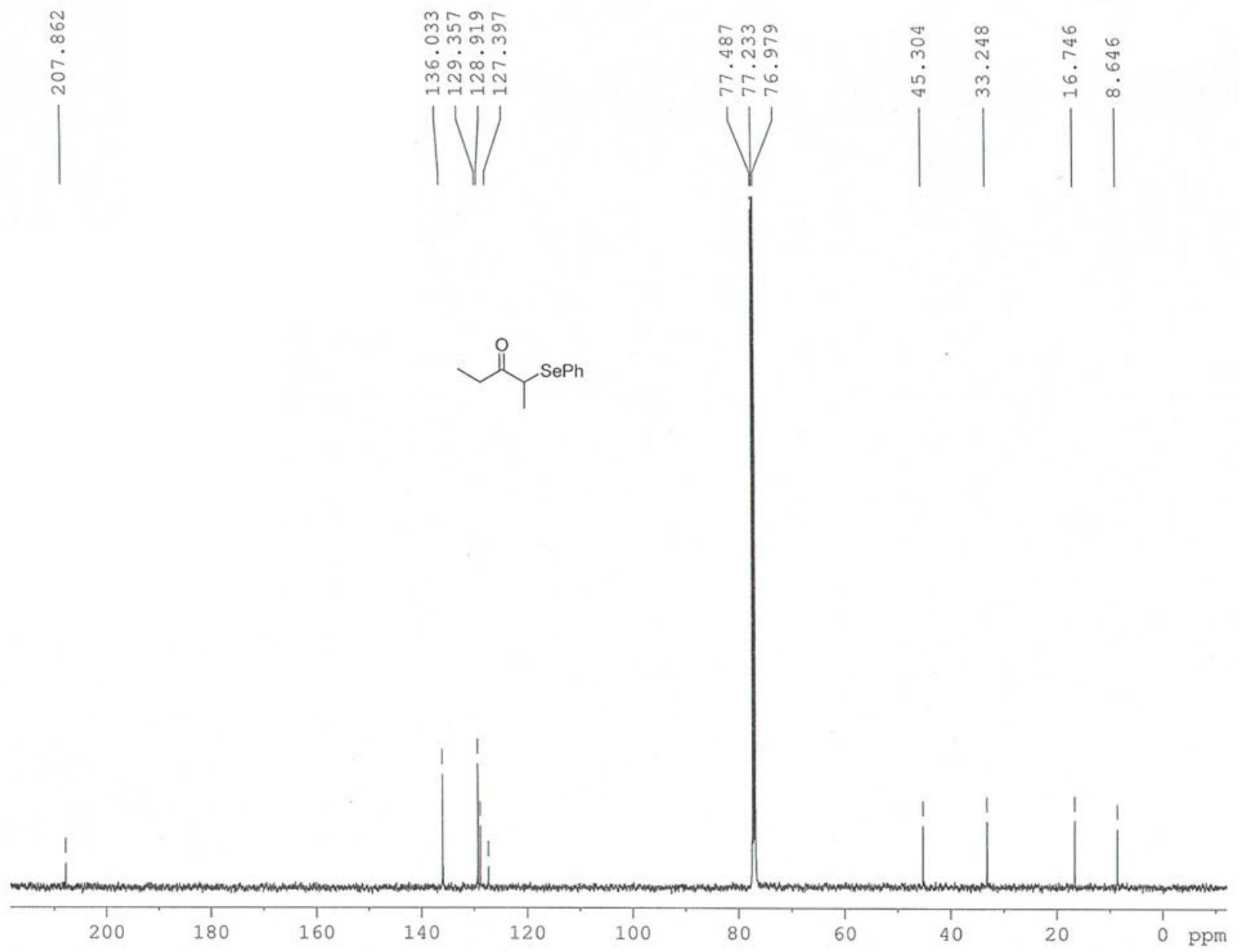




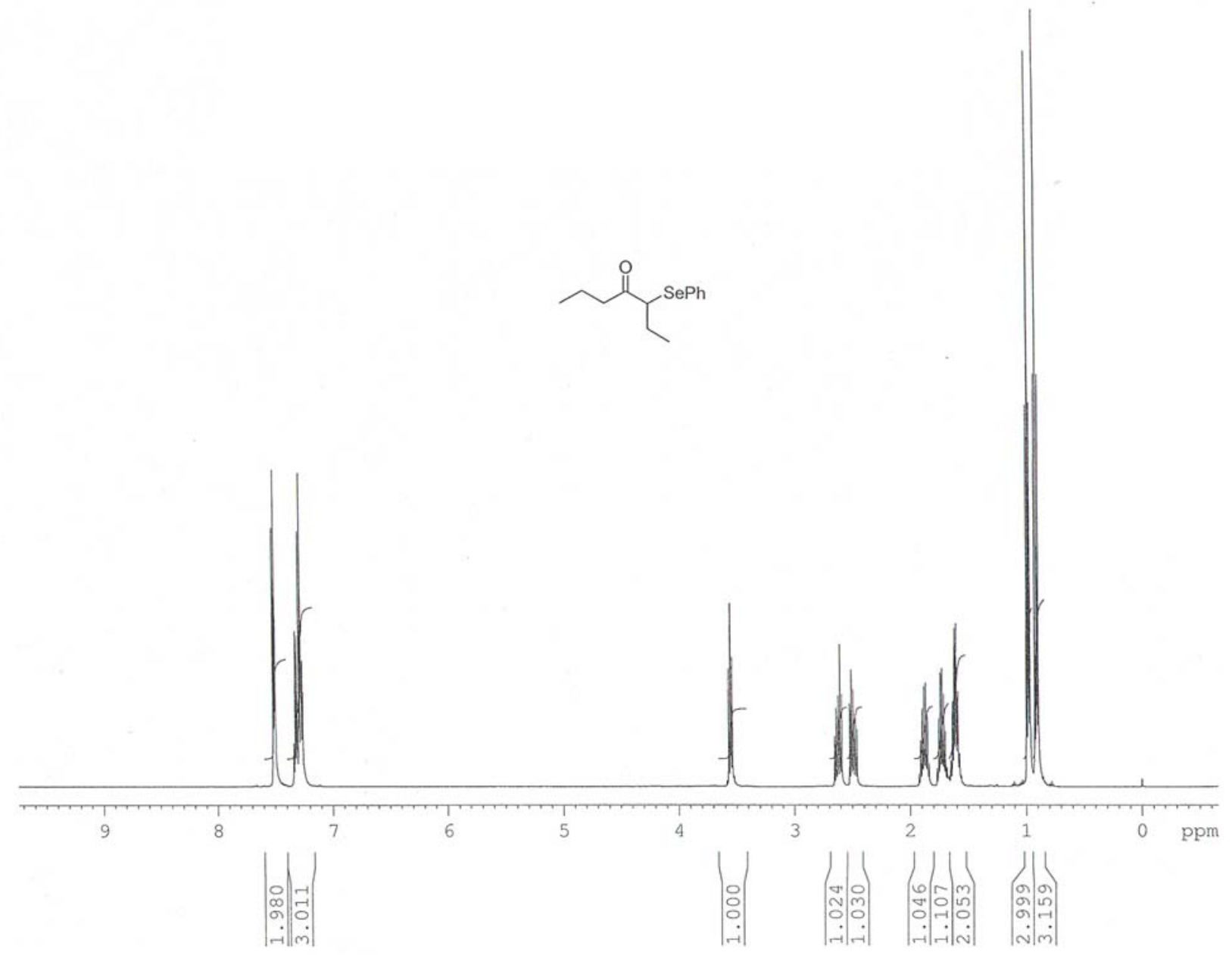




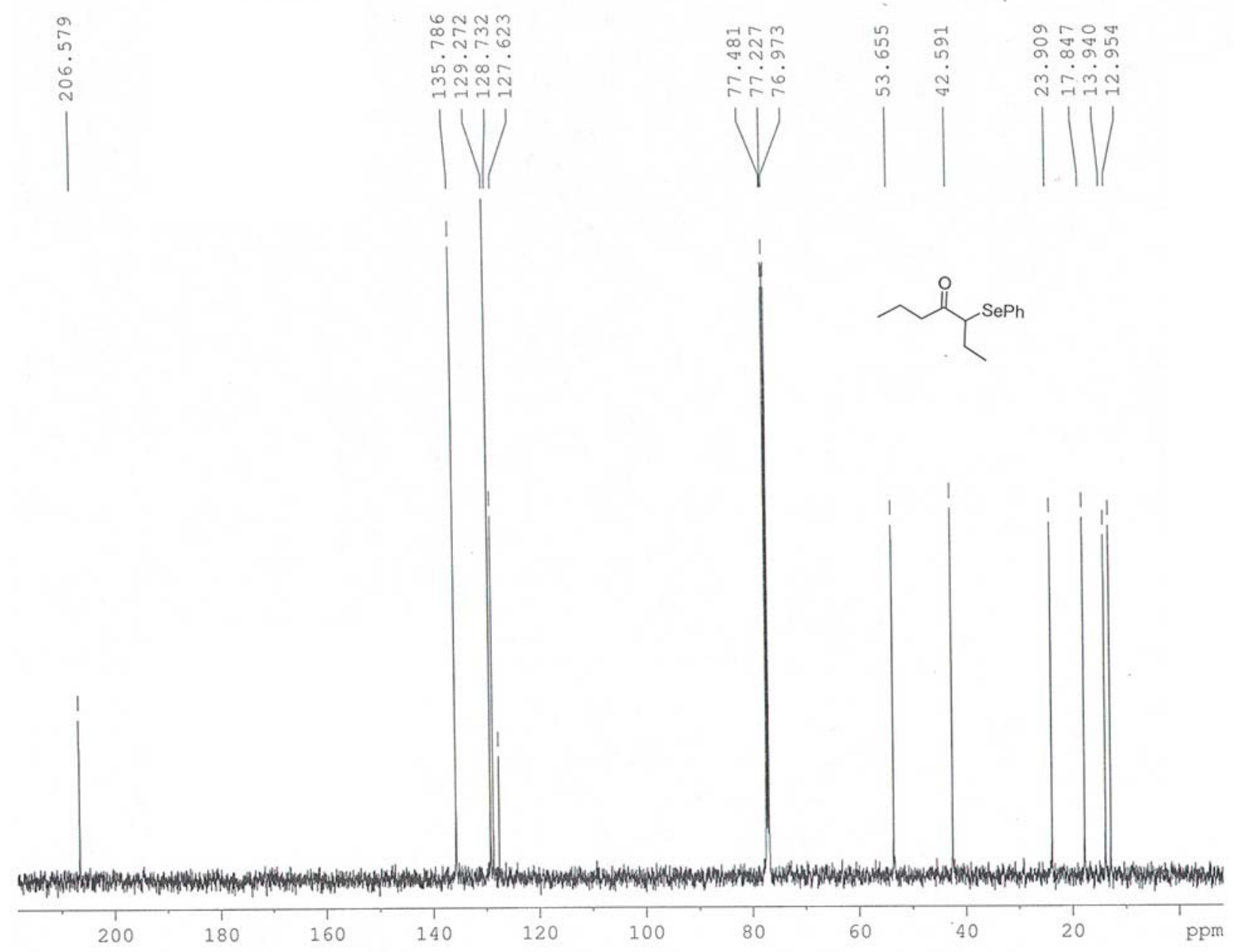




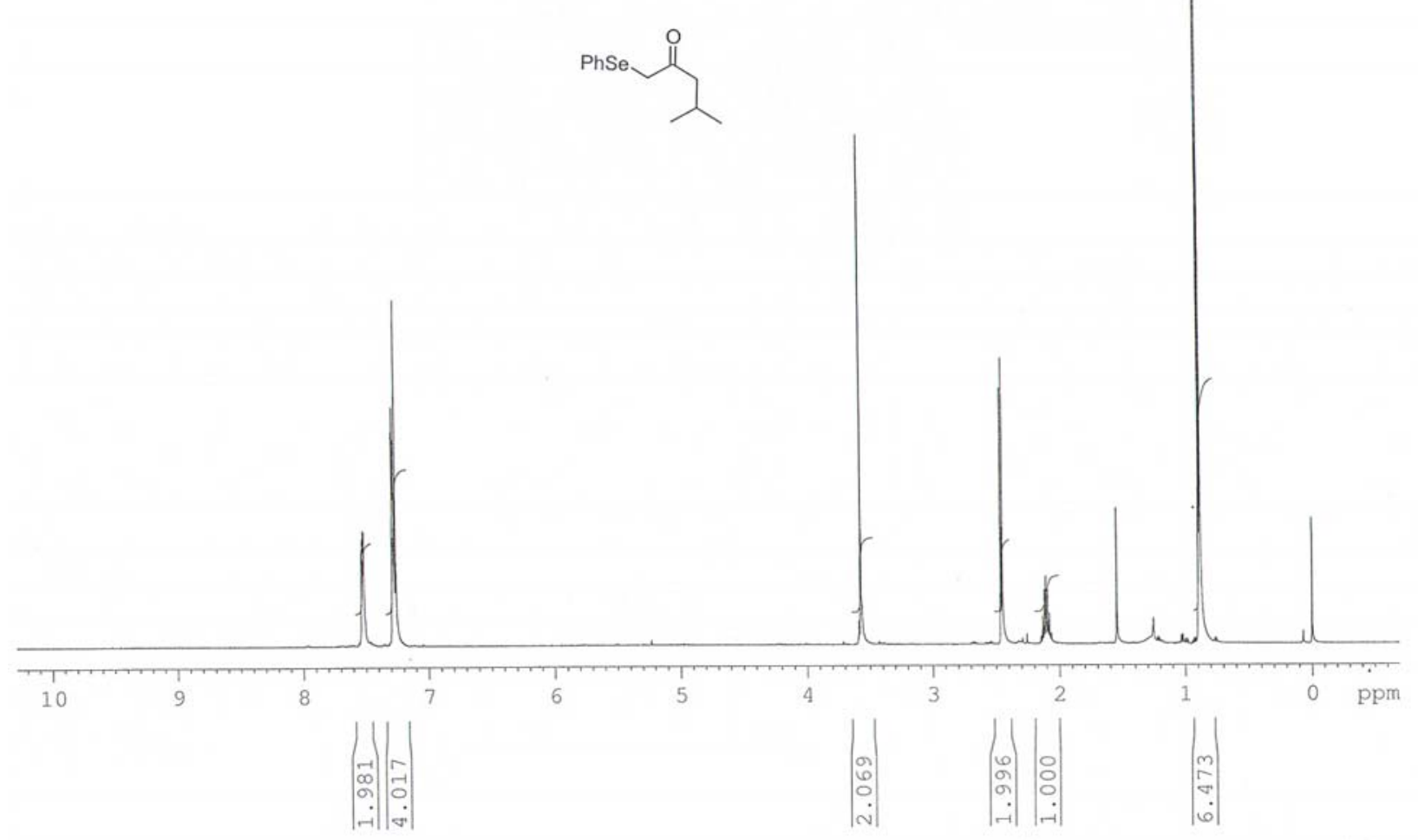

S40 


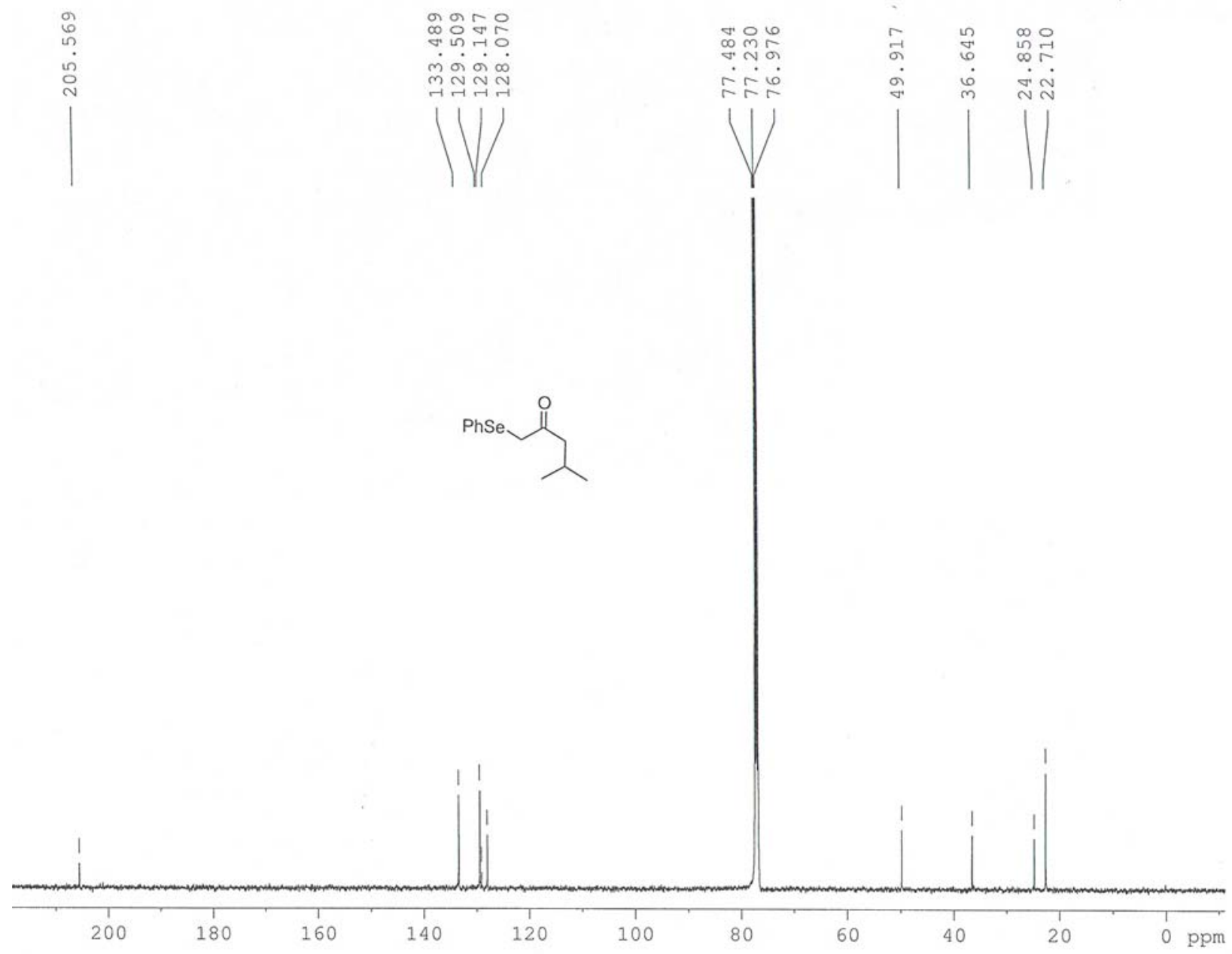




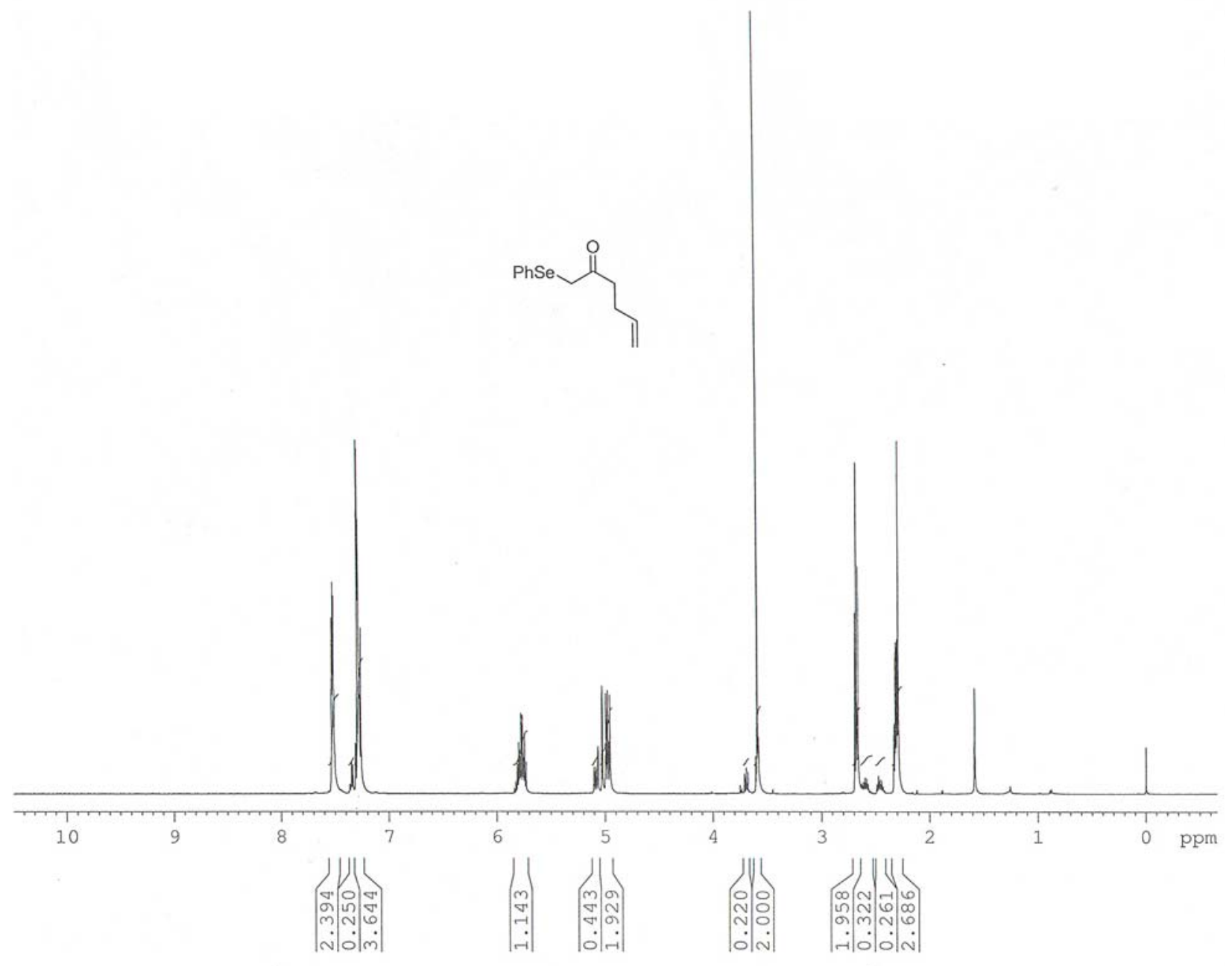




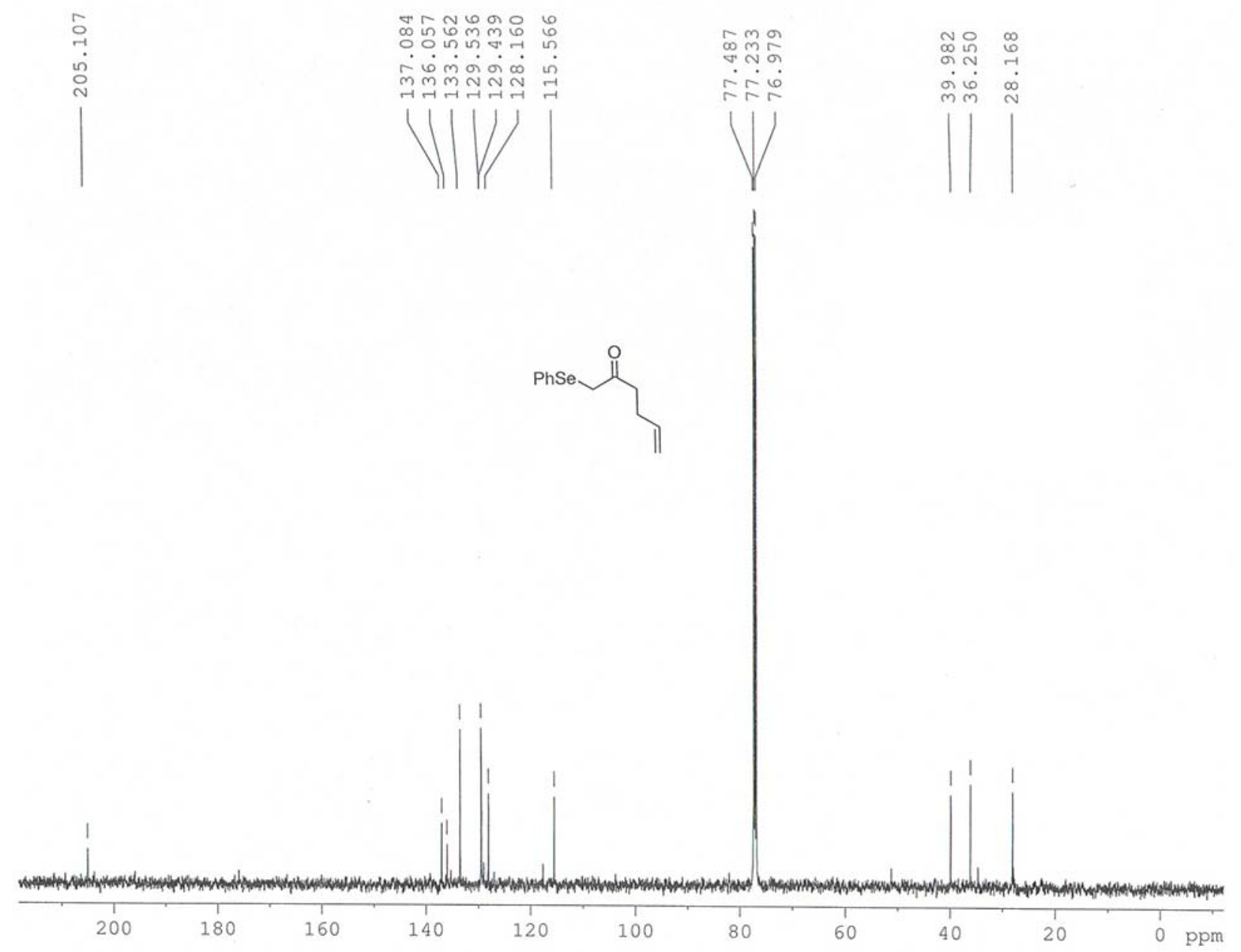




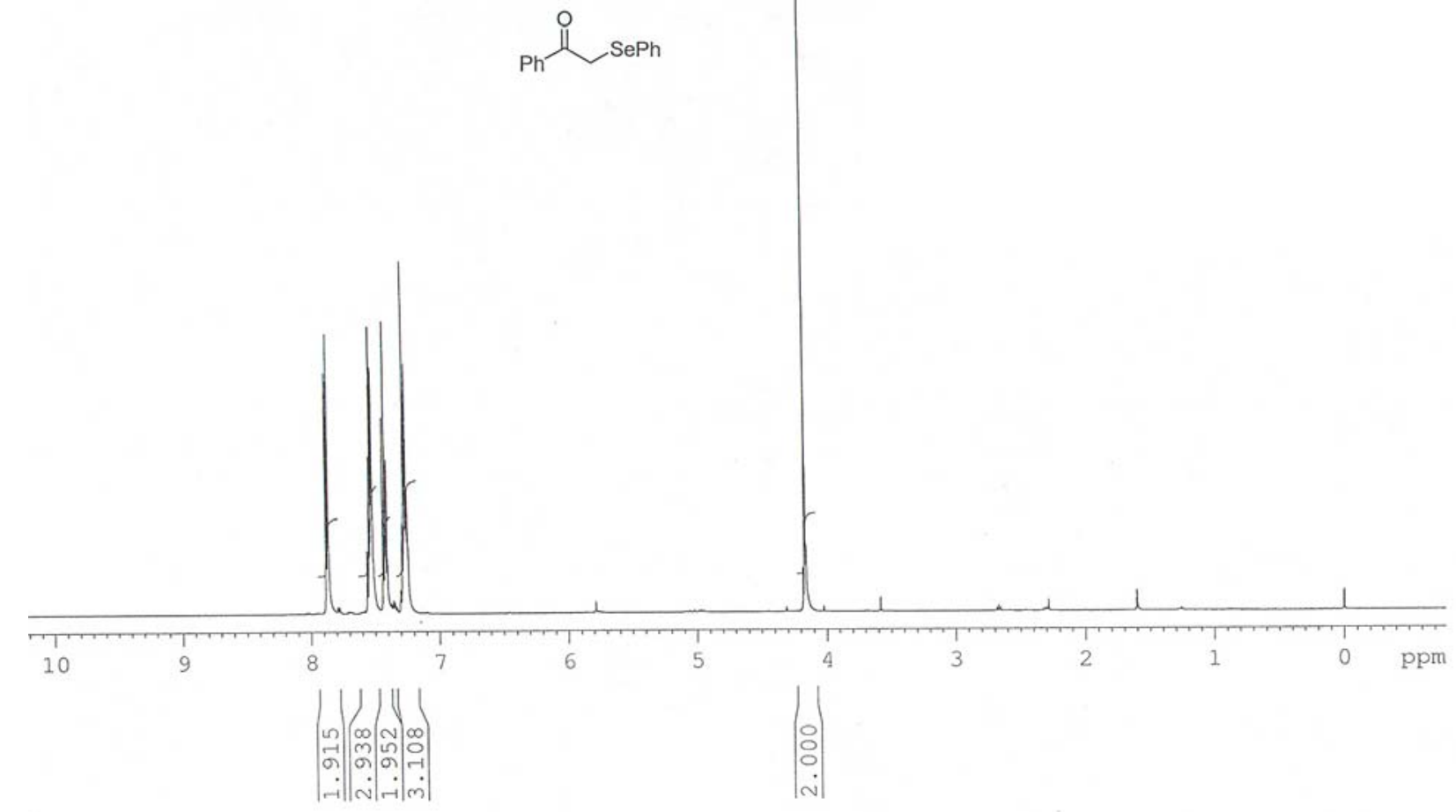




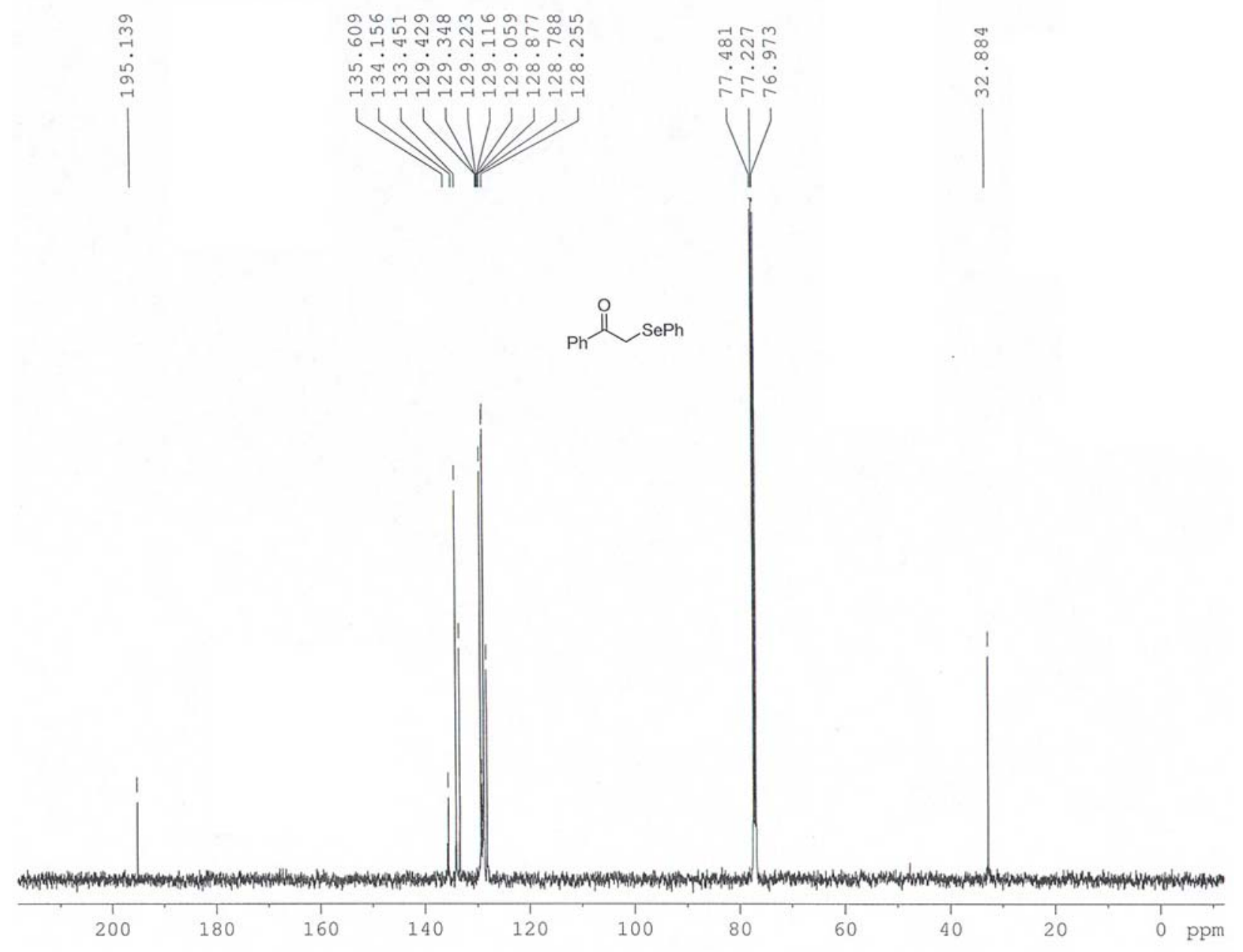




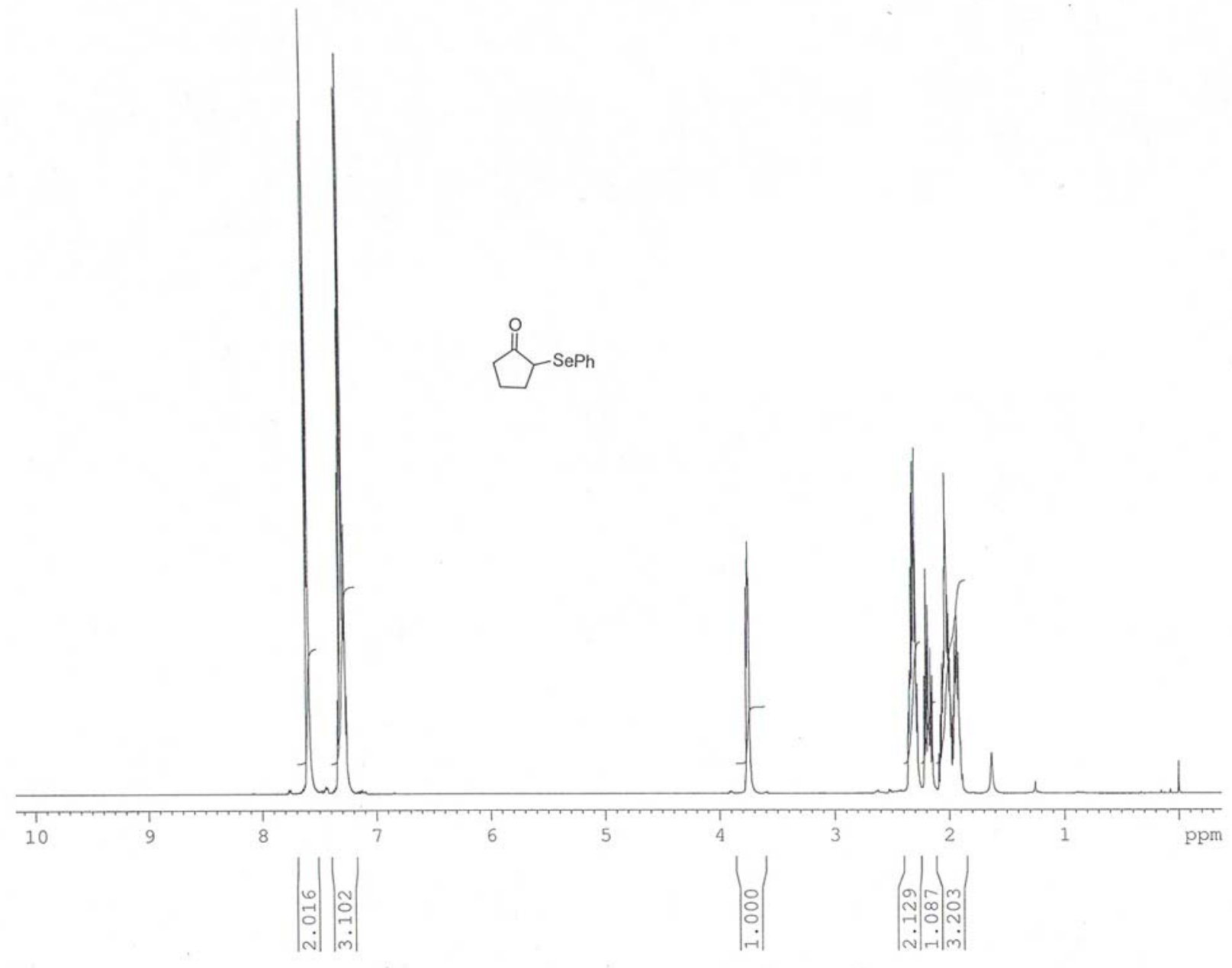




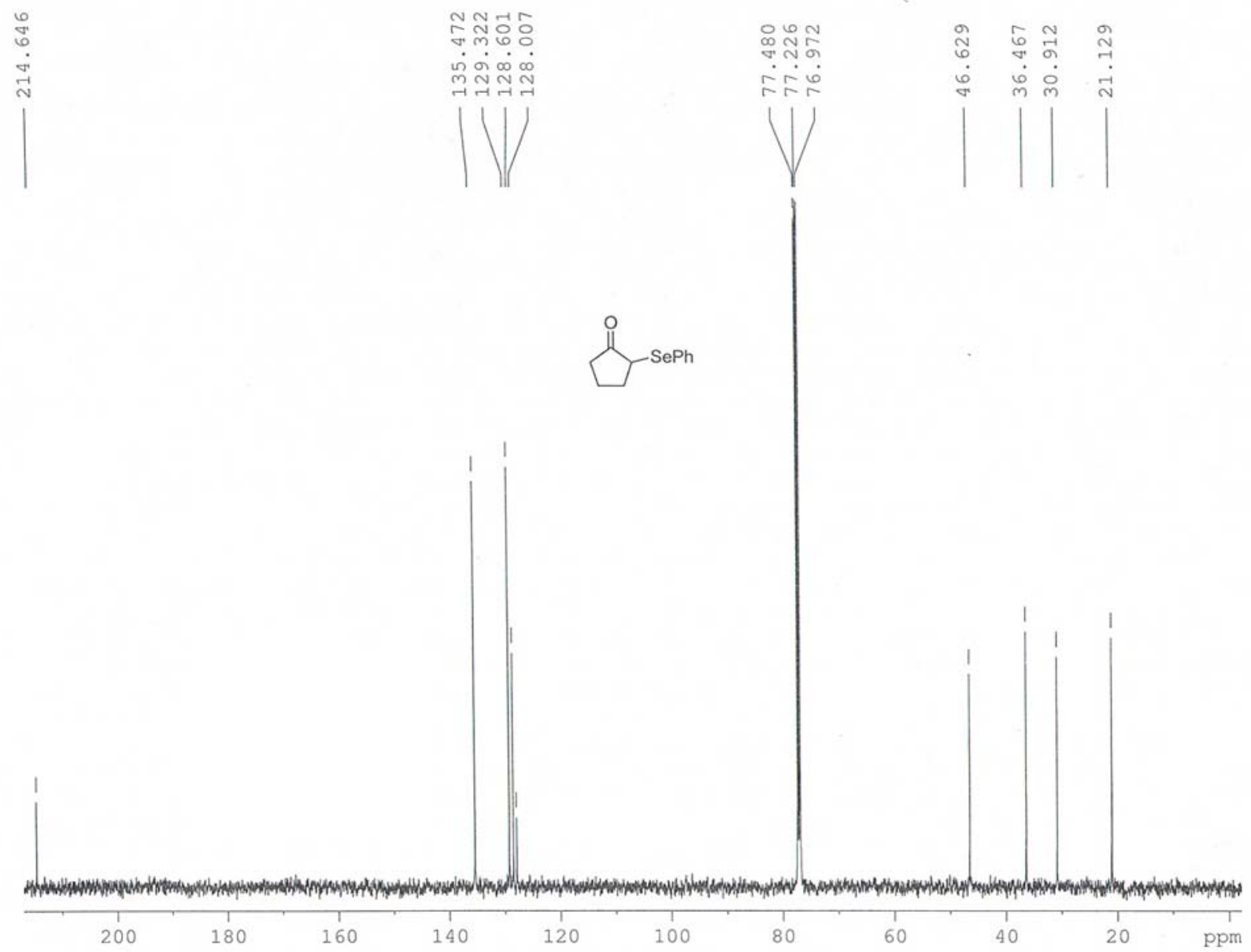




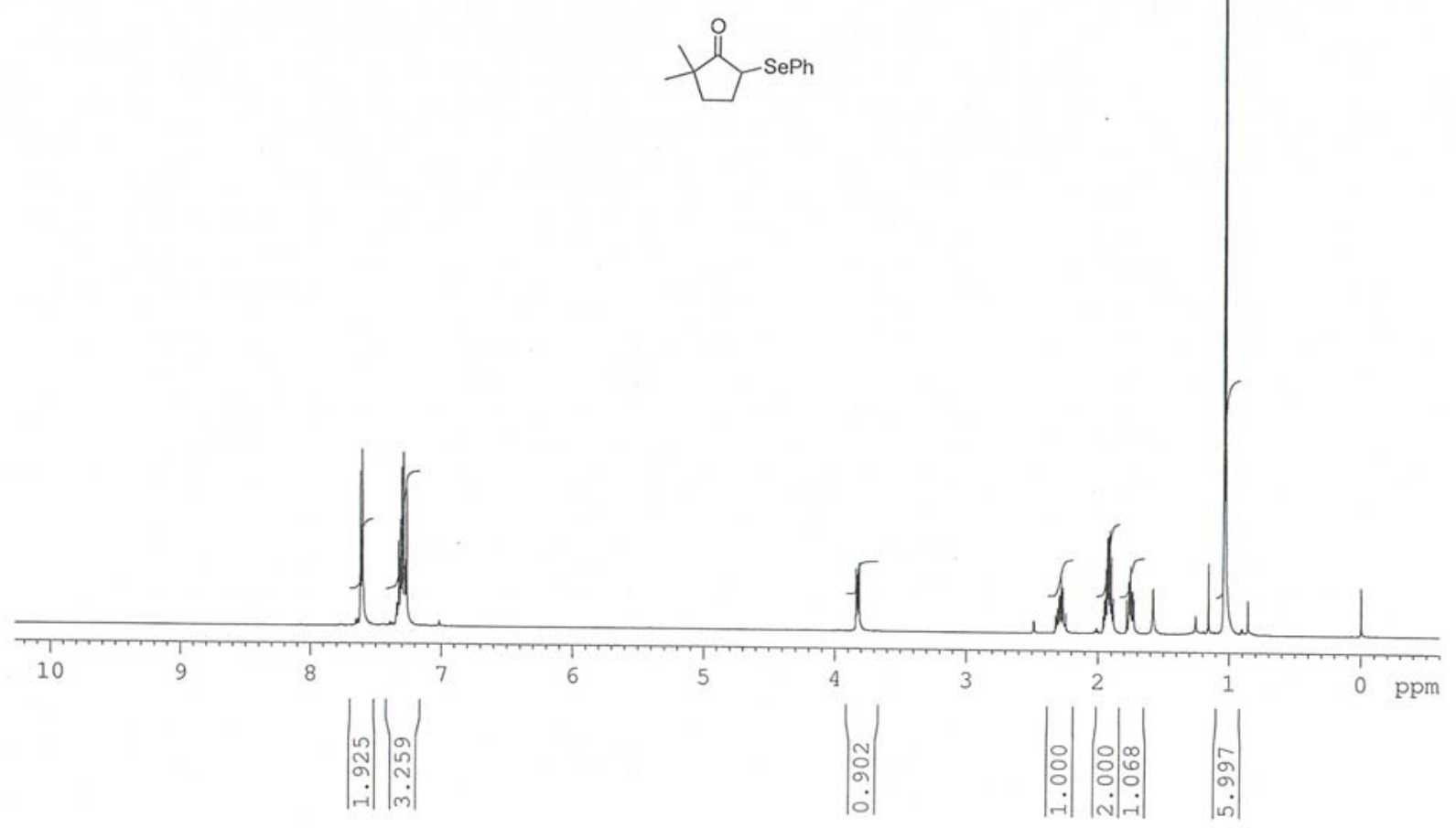




E

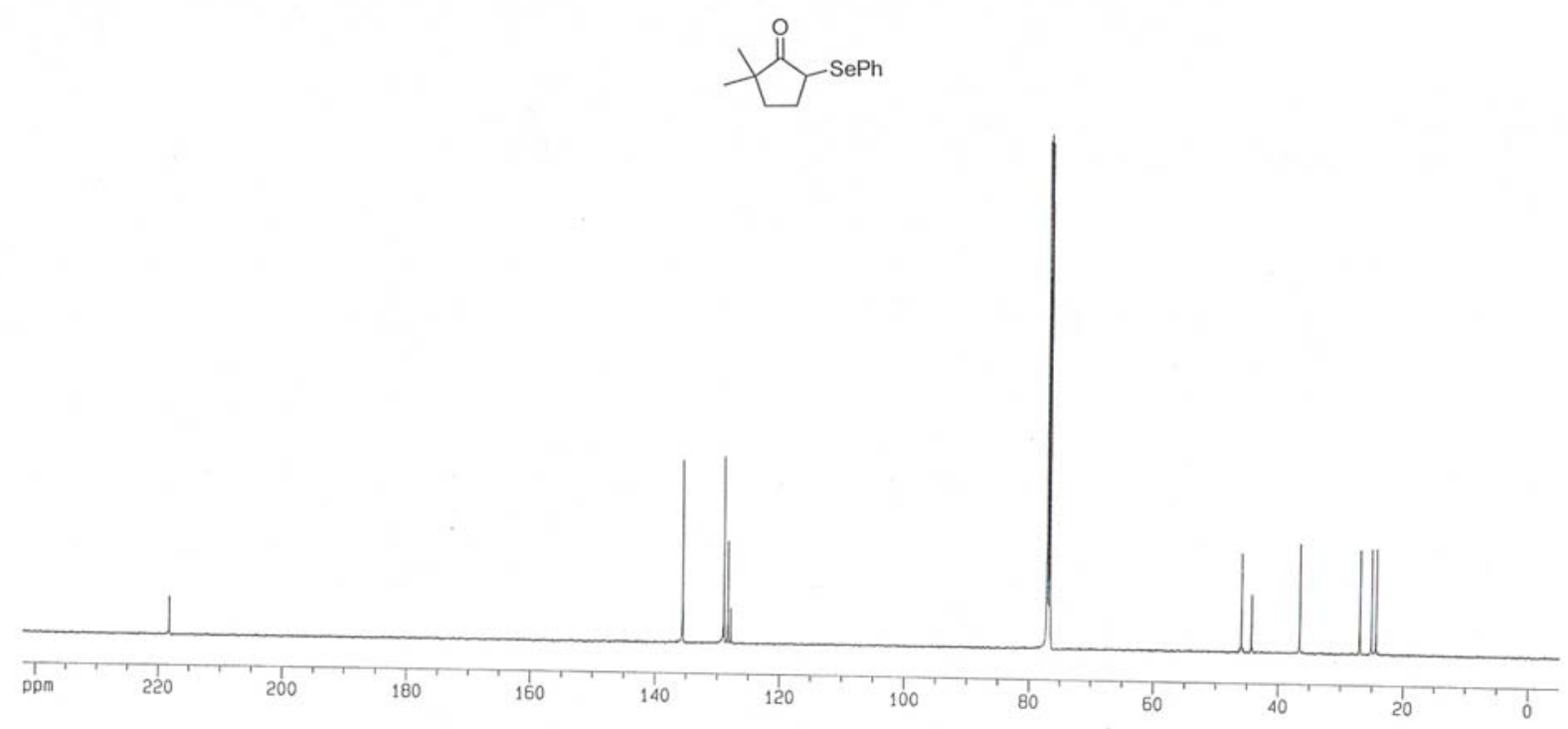




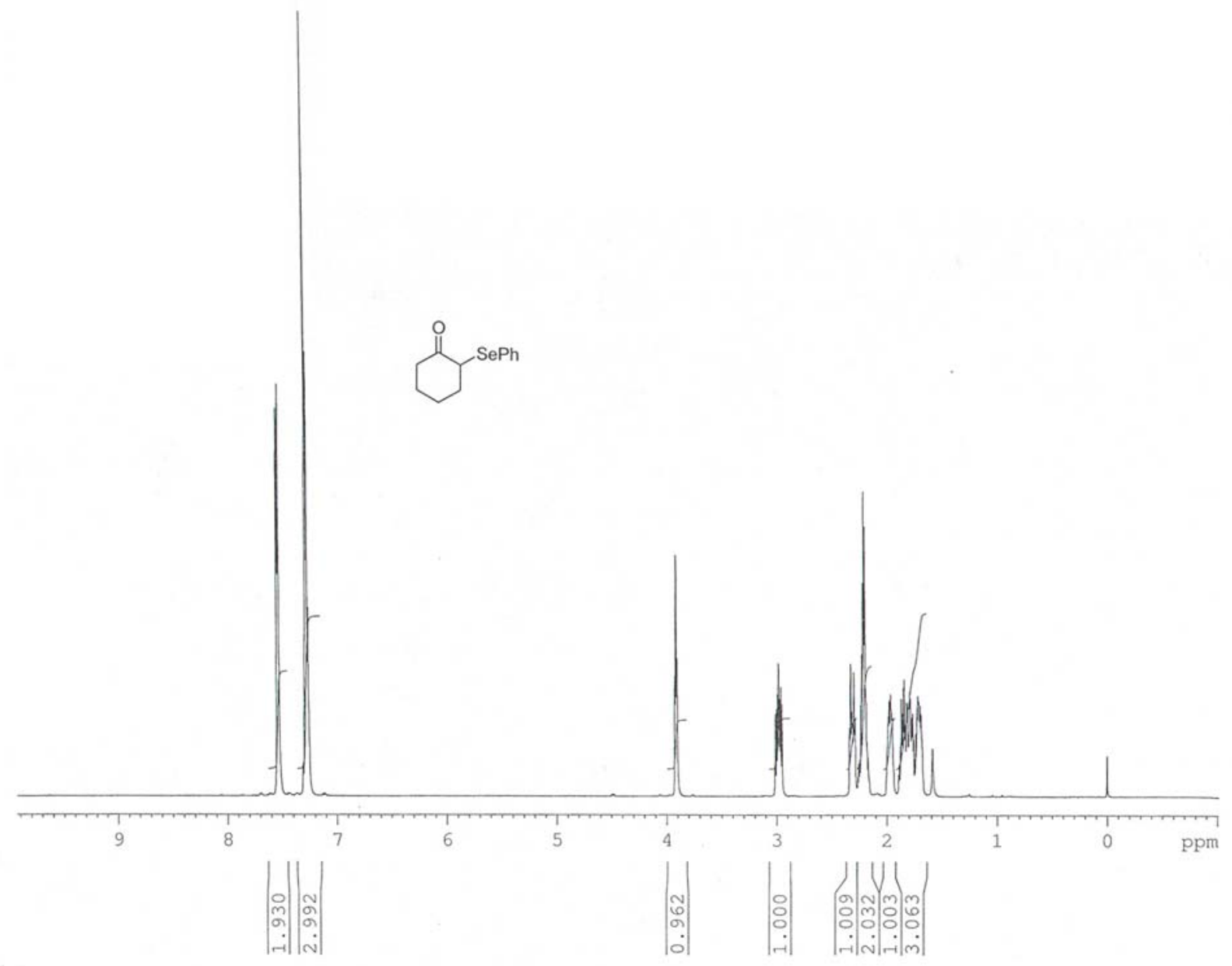




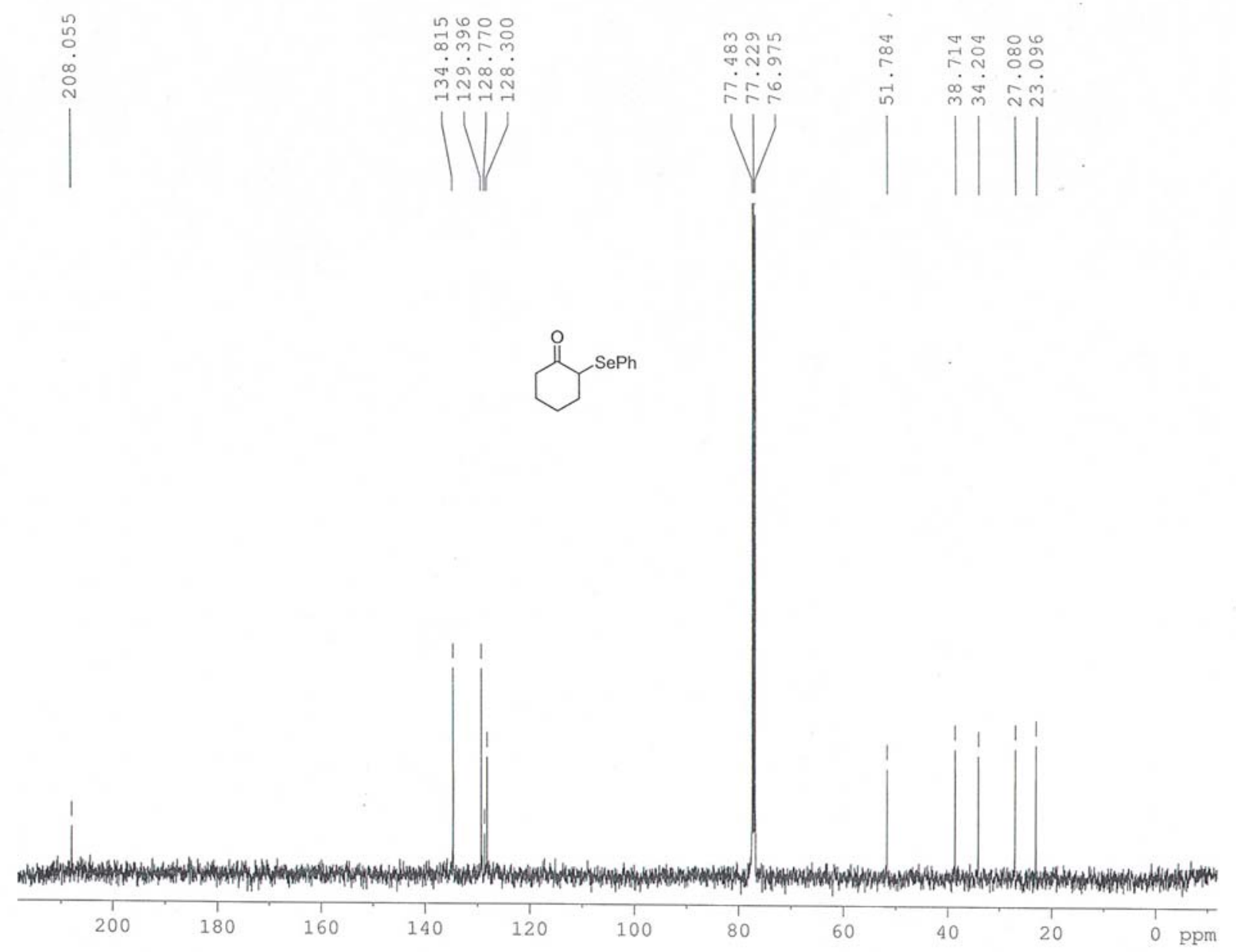




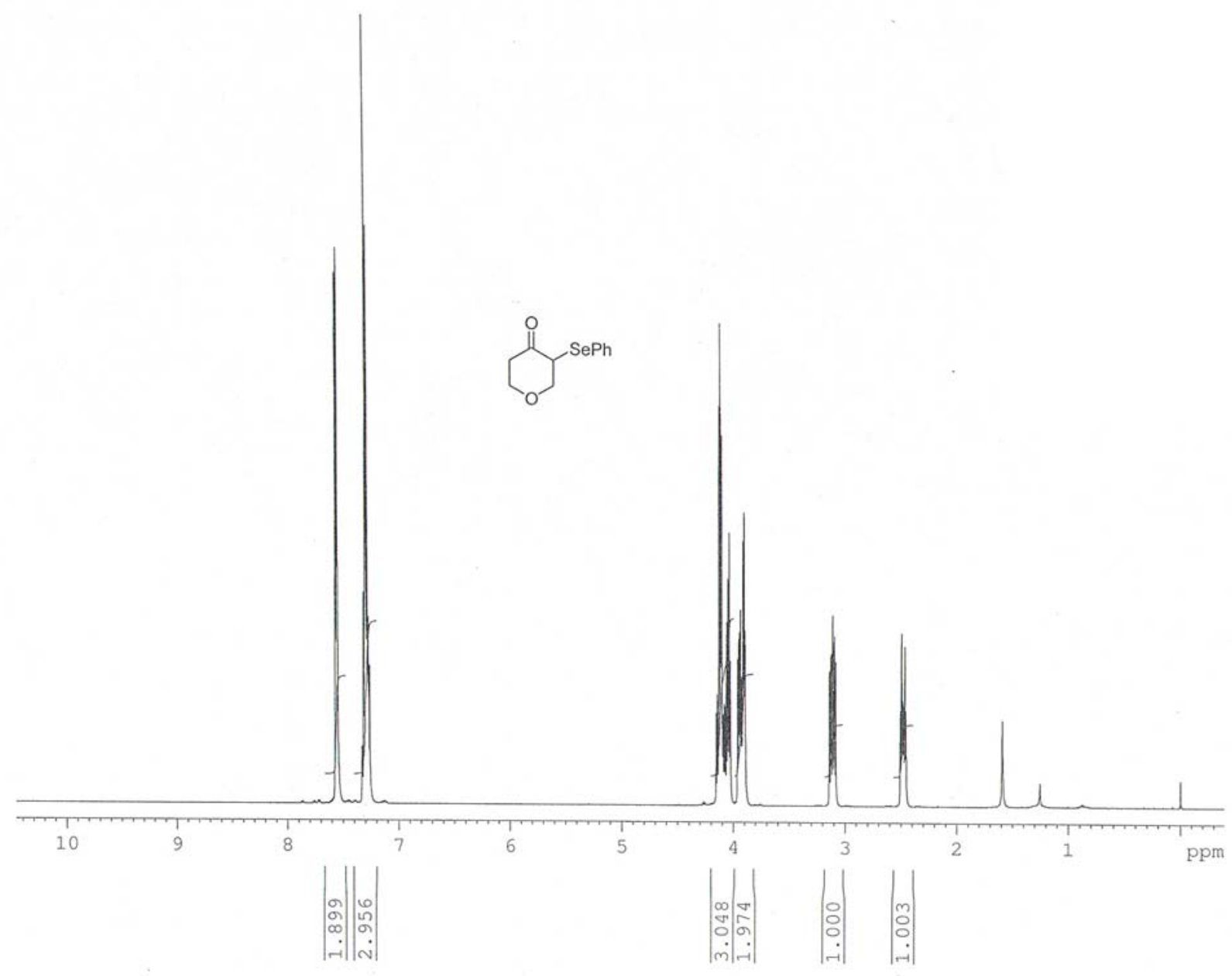




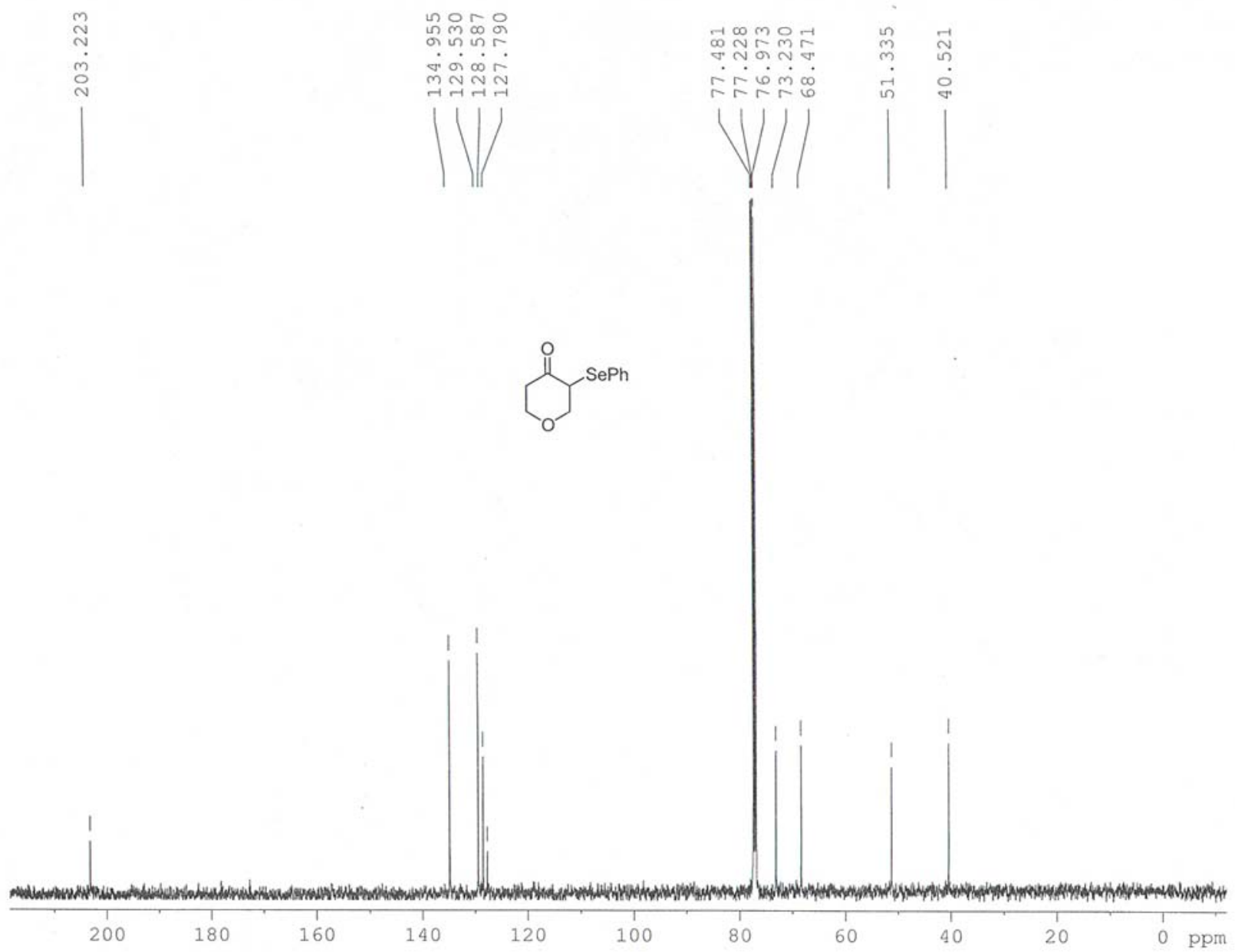




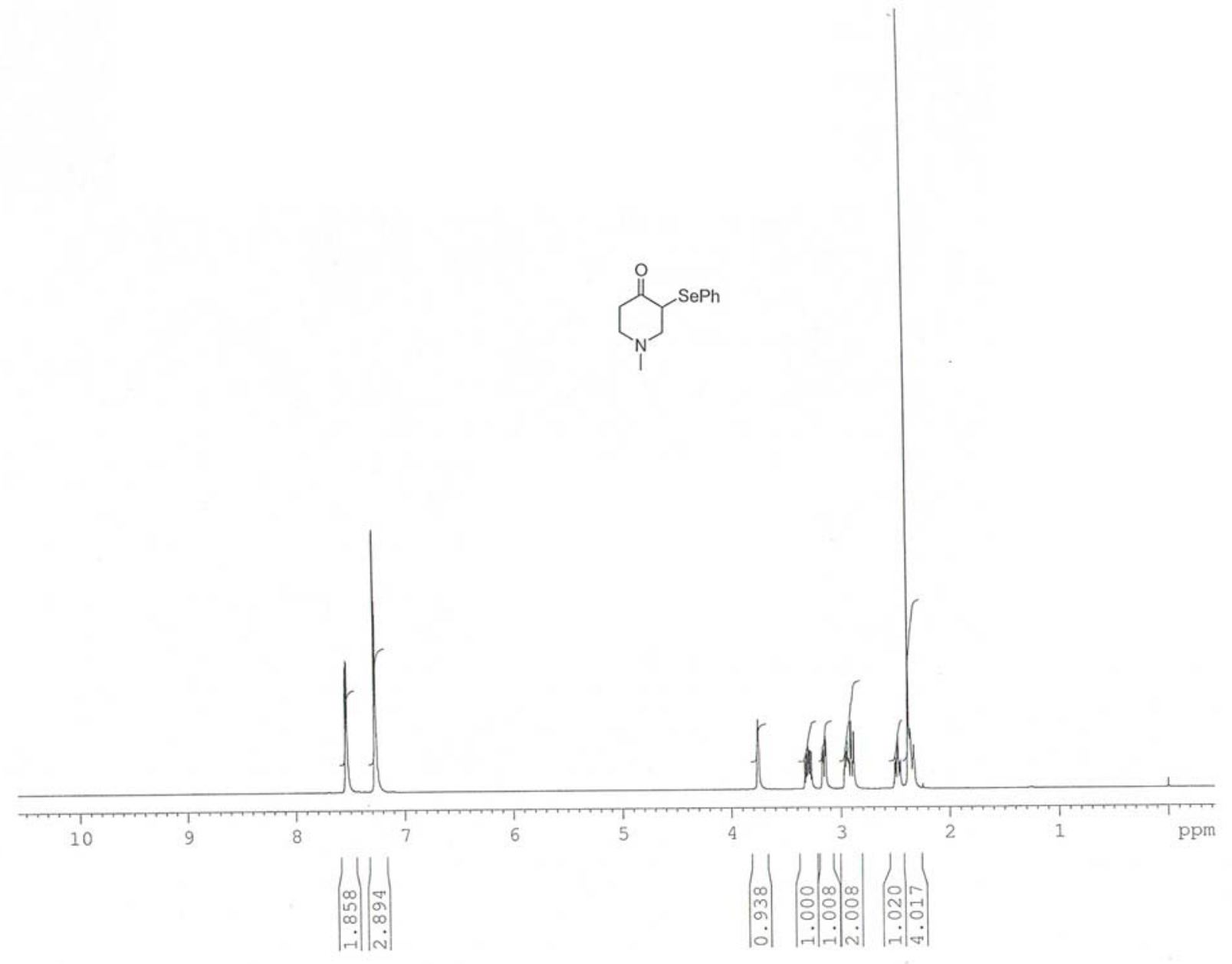




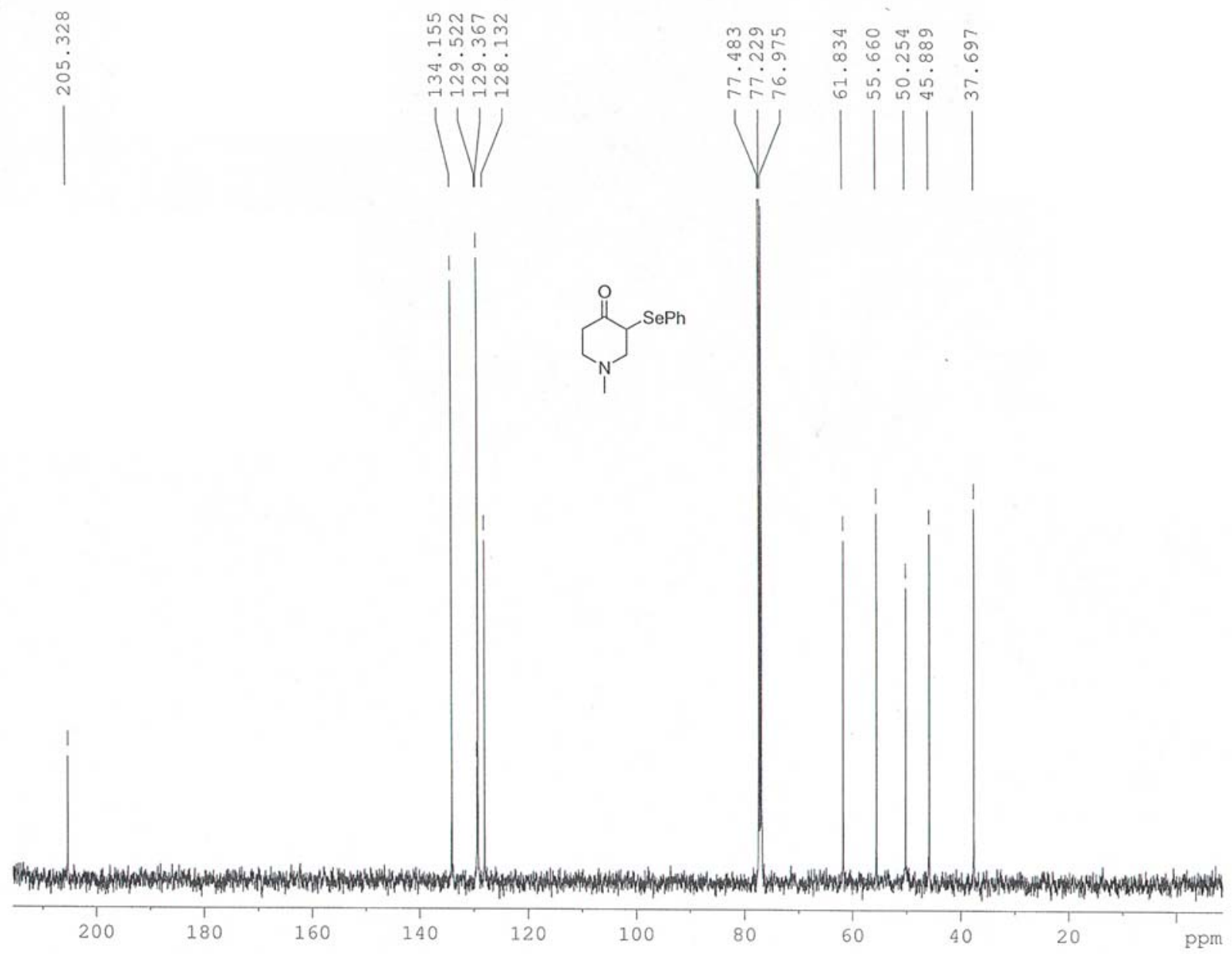




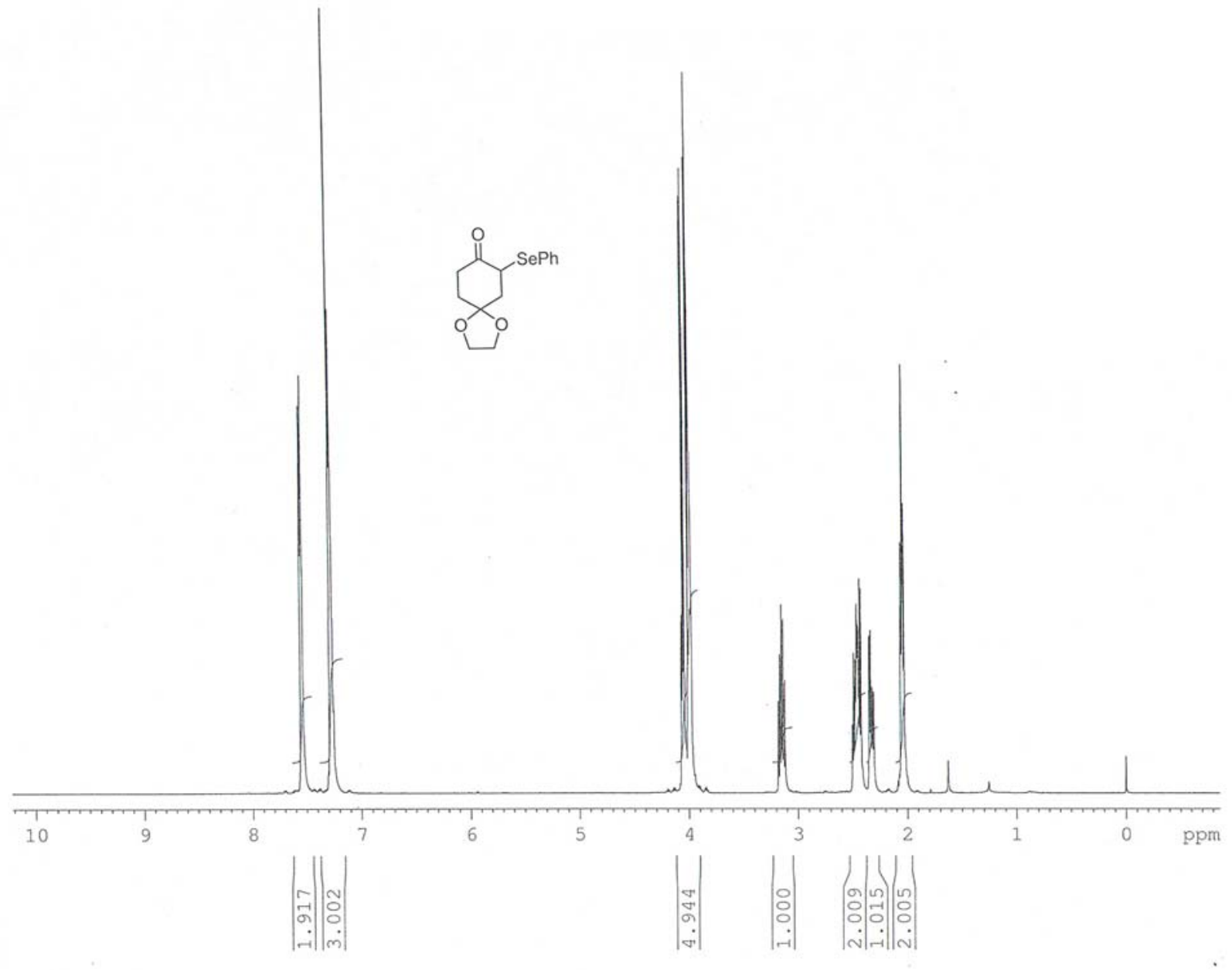




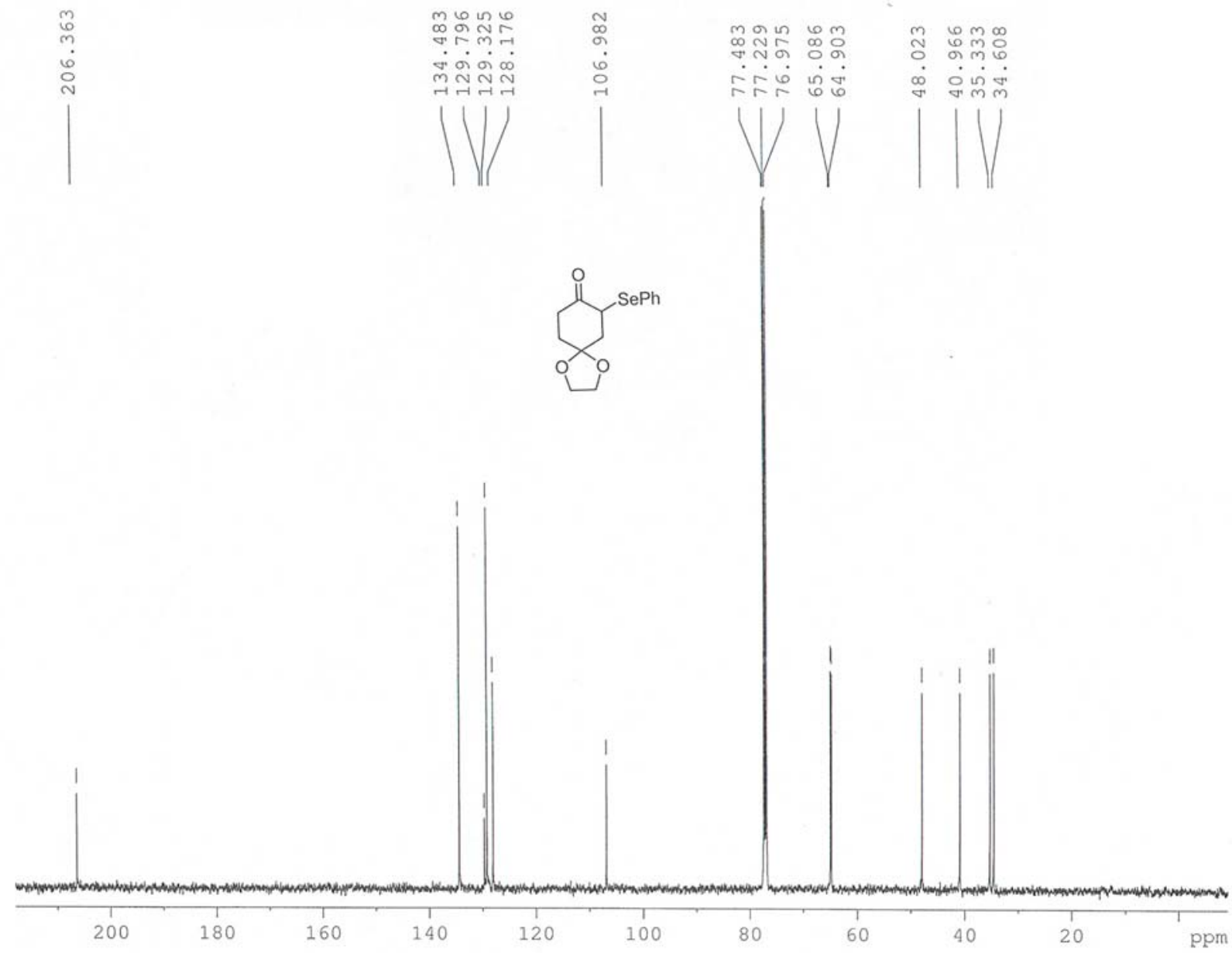




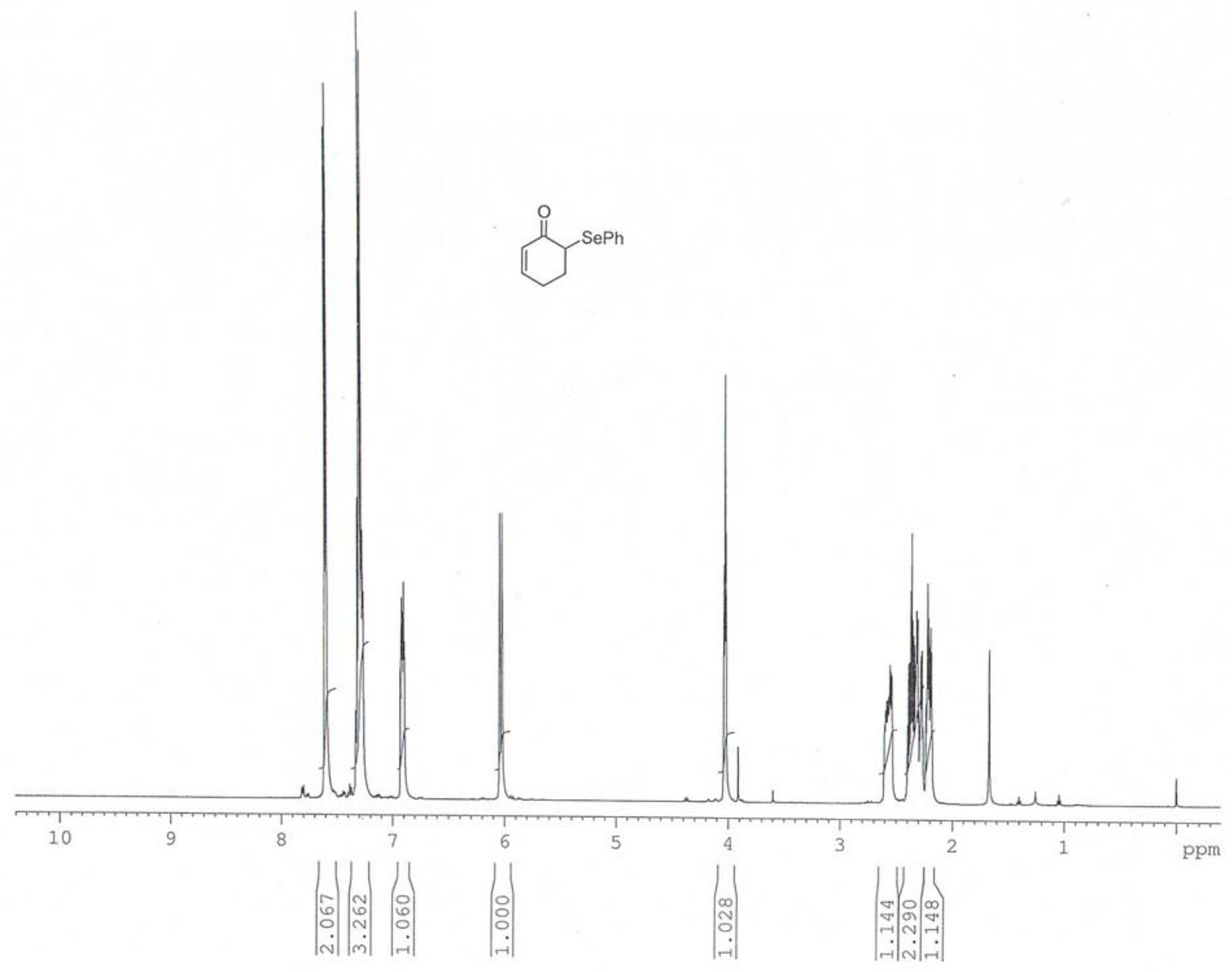




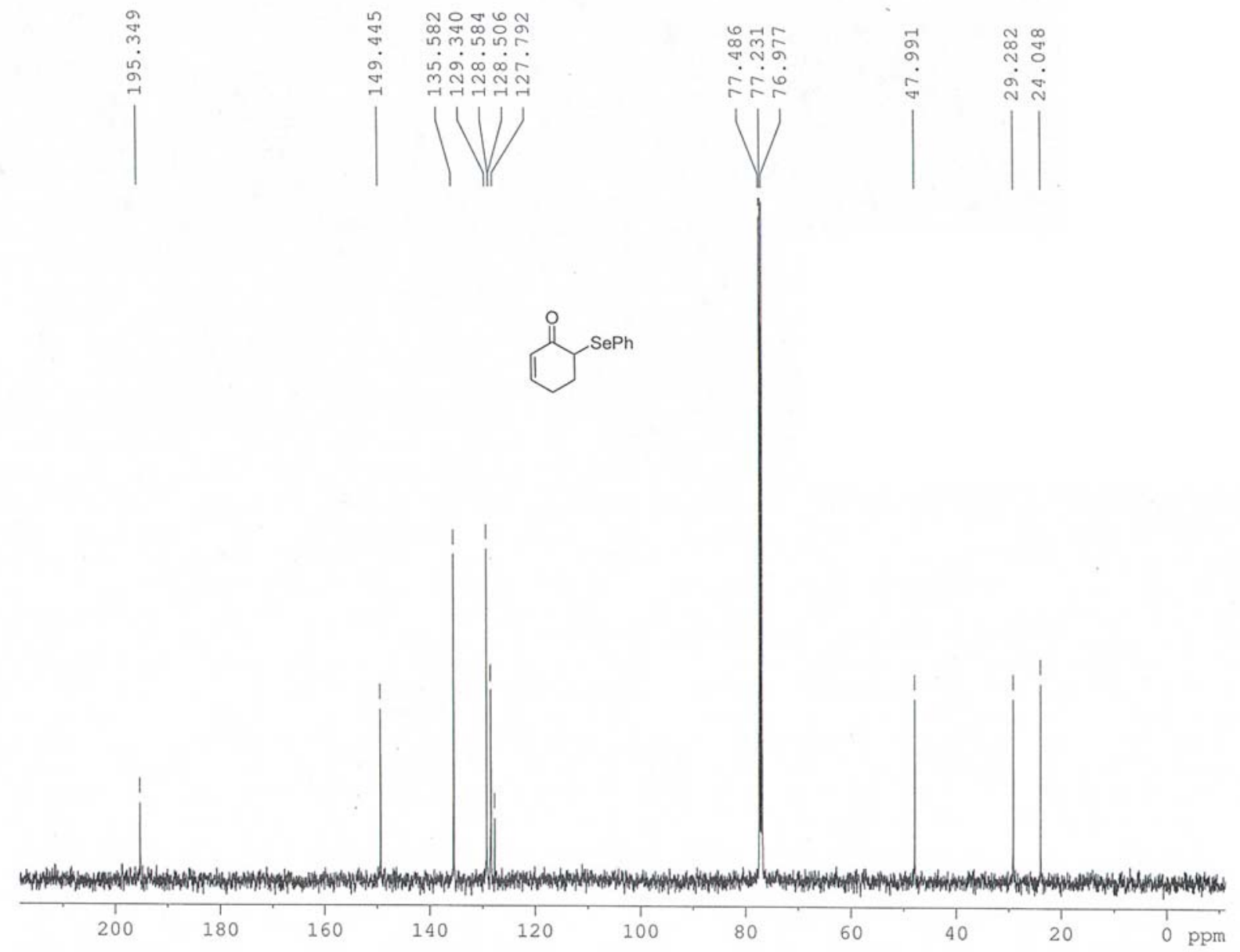




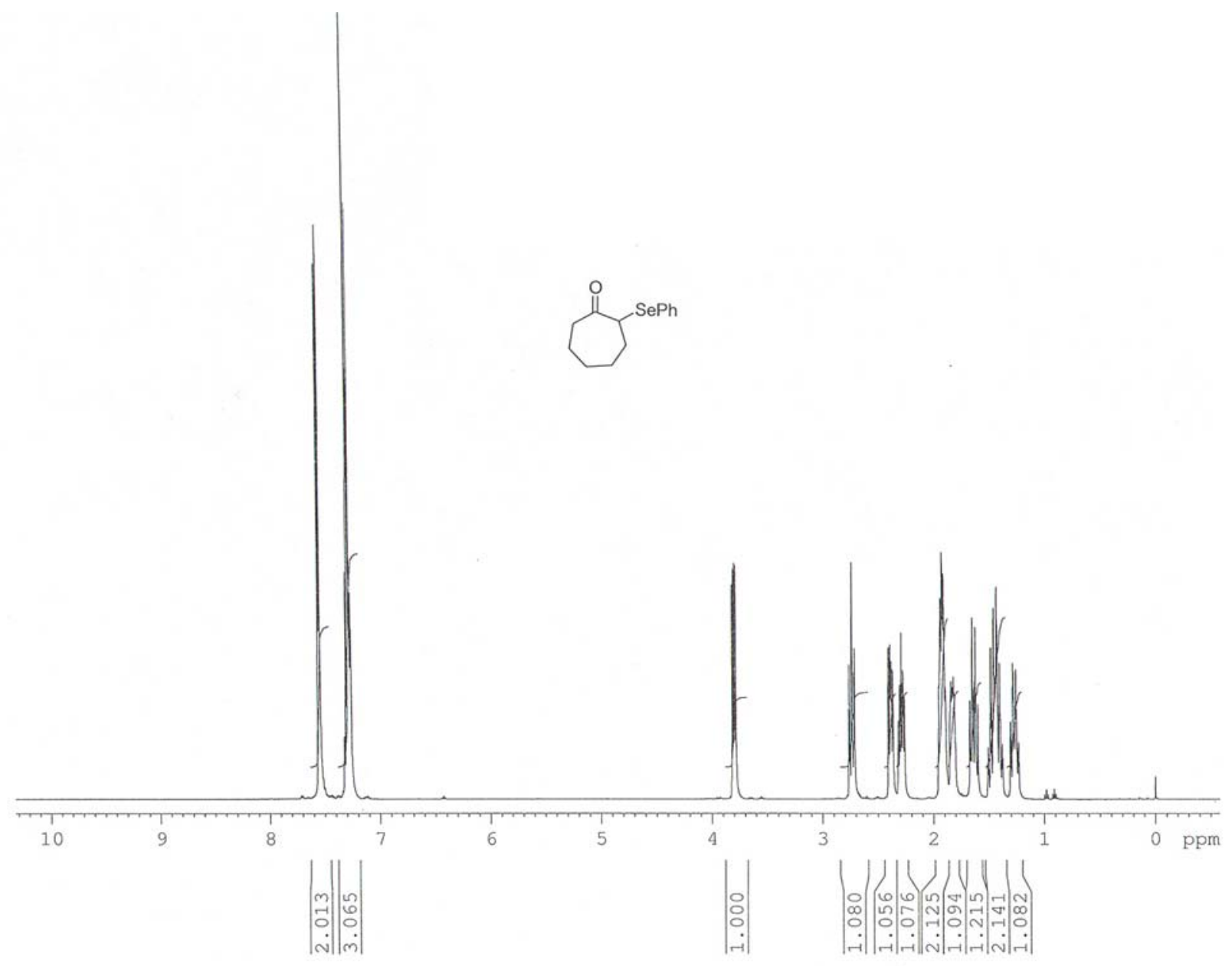




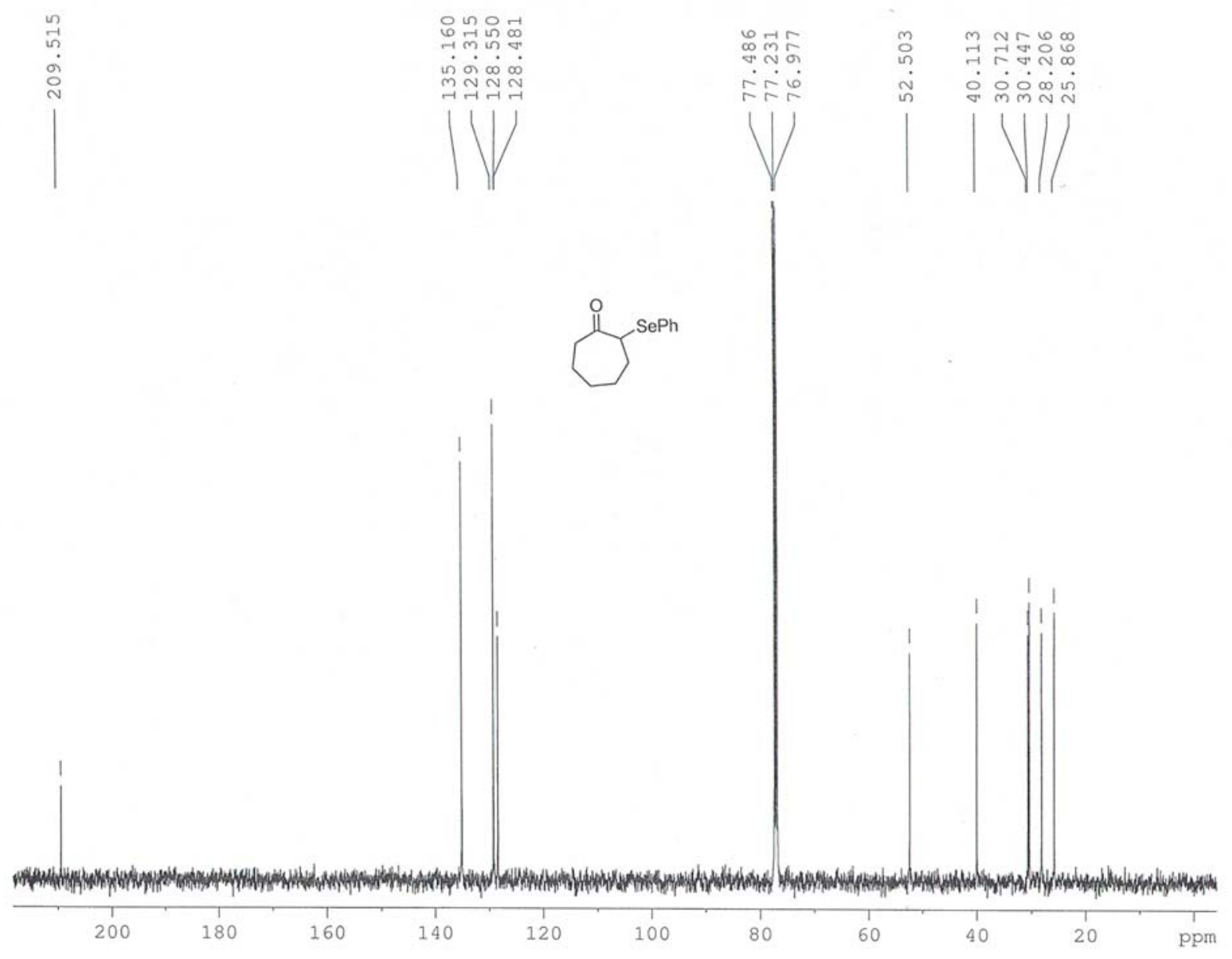

